University of Tennessee Health Science Center

UTHSC Digital Commons

\title{
Neuroanatomical Maps and Taste Reactivity to Sweet, Umami, and Bitter Taste in the PBN of C57BL/6J Mice
}

Jennifer Marie Saputra

University of Tennessee Health Science Center

Follow this and additional works at: https://dc.uthsc.edu/dissertations

Part of the Neurosciences Commons

\section{Recommended Citation}

Saputra, Jennifer Marie (http://orcid.org/0000-0002-7317-8307), "Neuroanatomical Maps and Taste Reactivity to Sweet, Umami, and Bitter Taste in the PBN of C57BL/6J Mice" (2016). Theses and Dissertations (ETD). Paper 401. http://dx.doi.org/10.21007/etd.cghs.2016.0413.

This Dissertation is brought to you for free and open access by the College of Graduate Health Sciences at UTHSC Digital Commons. It has been accepted for inclusion in Theses and Dissertations (ETD) by an authorized administrator of UTHSC Digital Commons. For more information, please contact jwelch30@uthsc.edu. 


\title{
Neuroanatomical Maps and Taste Reactivity to Sweet, Umami, and Bitter Taste in the PBN of C57BL/6J Mice
}

\begin{abstract}
Humans can distinguish at least five different taste qualities, sour, salty, bitter, sweet, and umami (the savory taste of certain amino acids). In neuroscience research, behavioral testing is used to measure the ability of rodents (including inbred mice) to discriminate between the different taste qualities. Taste reactivity and two-bottle preference are behavioral tests that are utilized to investigate different aspects of taste. These tests involve either voluntary or forced consumption of taste stimuli, respectively. Either test can be used to infer the preference and palatability of the stimulus consumed by an animal.

In order to understand the basis of taste behavior, one must understand the organization of the taste pathway. As an organism consumes a particular food or fluid, it first binds to or activates taste receptors or channels located inside taste buds found in the oral cavity. This transduction event then produces a cascade of neuronal activation via sensory nerves that innervate the taste buds -branches of three cranial nerves (VII, IX, and X). These cranial nerves then synapse centrally in the nucleus of the solitary tract (NST) where the relayed taste information is kept relatively segregated from visceral input (which arrives via cranial nerve $\mathrm{X}$ ).
\end{abstract}

From this point, the taste information is relayed to the parabrachial nucleus (PBN) in the pons, where the taste and visceral information now overlap. The PBN has not been studied as extensively as the NST in terms of taste representation, especially in regards to umami taste. A few recent studies have indicated that taste neurons in the PBN respond to sweet and synergistic umami (i.e. a combination of glutamate and a ribonucleotide) stimuli in a similar manner, providing a rationale for further study of the representation of these taste stimuli in this area.

Sweet and umami taste share a common G-protein-coupled taste receptor subunit, T1R3, that responds in combination with either T1R1 to transduce umami stimuli or T1R2 to transduce sweet stimuli. Aside from sharing a common taste receptor, previous studies using pharmacological manipulations, electrophysiology, conditioned taste aversion (CTA), and discrimination studies have shown a strong functional link between sweet and umami taste in rodents. Compounds found to be sweet taste inhibitors either entirely or partially block the nerve response to the prototypical umami stimulus monosodium glutamate (MSG), as well as a synergistic mixture of MSG combined with the cyclic nucleotide inosine monophosphate (IMP). When the epithelial sodium channel blocker amiloride is combined with MSG, both rats and mice have difficulty determining the difference between this umami stimulus and sucrose. Overall, it appears that some umami stimuli appear to be perceived as sucrose-like in rodents, which differs dramatically from the human perception of umami stimuli. Although umami taste has not been studied as comprehensively in mice as it has been in rats, it is important to investigate due to the widespread use of a variety of genetic mouse models in taste research. Along with using behavioral models, one might gauge the uniqueness of sweet and umami stimuli using an anatomical technique, such as visualization of the immediate early gene $c$-fos in PBN neurons. In fact, previous research has indicated stimulation with different taste qualities produces distinctive $c$-fos patterns in the PBN. For this current research study, my first hypothesis was that since previous studies suggested the similarity between sweet and umami compounds in C57BL/6J (B6) mice; stimuli of both taste qualities would produce similar levels of preference, consumption, and levels of taste reactivity behaviors. Secondly, I hypothesized that taste stimulation with either sweet (sucrose) or umami (monopotassium glutamate; MPG, or the synergistic mixture of MPG+IMP) stimuli would produce a similar $c$-fos expression pattern in sweet and umami stimuli, and this would also be distinct from the $c$-fos expression patterns elicited by both the bitter stimulus, quinine hydrochloride $(\mathrm{QHCl})$ and water. 
Overall, the preference tests revealed that both sucrose and umami stimuli (especially MSG+IMP) were preferred and consumed at a similarly high level in B6 mice. However, the taste reactivity test did not yield any insight into whether the sweet and umami taste stimuli were perceived as similar. However, taste reactivity to the bitter stimulus, $\mathrm{QHCl}$, was easily distinguishable from the other tested taste stimuli. Using $c$-fos immunohistochemistry to visualize neuronal activation, I then compared staining patterns of activation evoked by: water, QHCl, sucrose, saccharin, MPG, and MPG+IMP in subdivisions of the PBN in B6 mice, as well as a few other non-taste brainstem areas (locus coeruleus and mesencephalic nucleus of the trigeminal nerve). Results showed that quinine elicited significantly less $c$-fos positive nuclei in the entire dorsal lateral (DL) subnucleus compared to water. A few other significant effects of the tastant stimuli were found in the rostral portion of the waist, central lateral (CL), and DL PBN subnuclei, but distinct $c$-fos representations were not found for each stimulus tested. To determine if tastant effects might have been subtler in terms of cell density or patterning; and

therefore, could have been missed using normal cell counting methods, I decided to use a threedimensional mapping approach to examine $c$-fos expression in the PBN. Results of this new mapping approach suggest its potential usage in future studies.

\section{Document Type}

Dissertation

\section{Degree Name}

Doctor of Philosophy (PhD)

\section{Program}

Biomedical Sciences

\section{Research Advisor}

John D. Boughter, Jr., Ph.D.

\section{Keywords}

c-fos, PBN, taste reactivity, umami

\section{Subject Categories}

Medical Sciences | Medicine and Health Sciences | Neurosciences 
Neuroanatomical Maps and Taste Reactivity to Sweet, Umami, and Bitter Taste in the PBN of C57BL/6J Mice

\author{
A Dissertation \\ Presented for \\ The Graduate Studies Council \\ The University of Tennessee \\ Health Science Center
}

\begin{abstract}
In Partial Fulfillment
Of the Requirements for the Degree

Doctor of Philosophy

From The University of Tennessee
\end{abstract}

By

Jennifer Marie Saputra

May 2016 
Copyright (C) 2016 by Jennifer Marie Saputra.

All rights reserved. 


\section{DEDICATION}

I would like to dedicate this dissertation to my parents, Robert and Marie Paxson, and my husband, Leslie Saputra. To my parents- you have always been there for me and led me down the journey of my life. You both have encouraged me, loved me unconditionally, and helped me to realize my dreams. To my husband- you have walked beside me through my entire journey through graduate school, and you have supported and loved me unconditionally, even through the hard and messy times. I am incredibly grateful to all three of you. 


\section{ACKNOWLEDGMENTS}

I would like to acknowledge and thank my mentor, Dr. John Boughter, for his patience, time, expertise, advice, the many opportunities to attend conferences and present my research, and support that I have received over the years.

I would like to acknowledge and thank my committee members, Dr. Elberger, Dr. Fitzgerald, Dr. Hamre, and Dr. Steketee for all of your assistance, advice, and support.

I would like to acknowledge my past and current lab colleagues, Dr. Rebecca Glatt, Nick Saites, and Dr. Lianyi Lu for all their help, support, and friendship over the years. I would like to thank Zachary Goldsmith for quantifying the taste reactivity behaviors from the video recordings. I would like to thank Robert Paxson for his assistance with statistics. I would like to thank Dr. Kenichi Tokita for his assistance with the PBN and behavioral techniques. Also, I would like to thank Dr. Kenichi Tokita and Dr. Lianyi Lu for their help with teaching me the surgical techniques needed. I would like to thank Dr. Armstrong for teaching me how to use the Neurolucida software and microscope and for help in troubleshooting many issues. Lastly, I would also like to thank the Reiner lab for their assistance with immunohistochemistry. 


\begin{abstract}
Humans can distinguish at least five different taste qualities, sour, salty, bitter, sweet, and umami (the savory taste of certain amino acids). In neuroscience research, behavioral testing is used to measure the ability of rodents (including inbred mice) to discriminate between the different taste qualities. Taste reactivity and two-bottle preference are behavioral tests that are utilized to investigate different aspects of taste. These tests involve either voluntary or forced consumption of taste stimuli, respectively. Either test can be used to infer the preference and palatability of the stimulus consumed by an animal.

In order to understand the basis of taste behavior, one must understand the organization of the taste pathway. As an organism consumes a particular food or fluid, it first binds to or activates taste receptors or channels located inside taste buds found in the oral cavity. This transduction event then produces a cascade of neuronal activation via sensory nerves that innervate the taste buds -branches of three cranial nerves (VII, IX, and X). These cranial nerves then synapse centrally in the nucleus of the solitary tract (NST) where the relayed taste information is kept relatively segregated from visceral input (which arrives via cranial nerve X). From this point, the taste information is relayed to the parabrachial nucleus (PBN) in the pons, where the taste and visceral information now overlap. The PBN has not been studied as extensively as the NST in terms of taste representation, especially in regards to umami taste. A few recent studies have indicated that taste neurons in the PBN respond to sweet and synergistic umami (i.e. a combination of glutamate and a ribonucleotide) stimuli in a similar manner, providing a rationale for further study of the representation of these taste stimuli in this area.
\end{abstract}

Sweet and umami taste share a common G-protein-coupled taste receptor subunit, T1R3, that responds in combination with either T1R1 to transduce umami stimuli or T1R2 to transduce sweet stimuli. Aside from sharing a common taste receptor, previous studies using pharmacological manipulations, electrophysiology, conditioned taste aversion (CTA), and discrimination studies have shown a strong functional link between sweet and umami taste in rodents. Compounds found to be sweet taste inhibitors either entirely or partially block the nerve response to the prototypical umami stimulus monosodium glutamate (MSG), as well as a synergistic mixture of MSG combined with the cyclic nucleotide inosine monophosphate (IMP). When the epithelial sodium channel blocker amiloride is combined with MSG, both rats and mice have difficulty determining the difference between this umami stimulus and sucrose. Overall, it appears that some umami stimuli appear to be perceived as sucrose-like in rodents, which differs dramatically from the human perception of umami stimuli. Although umami taste has not been studied as comprehensively in mice as it has been in rats, it is important to investigate due to the widespread use of a variety of genetic mouse models in taste research. Along with using behavioral models, one might gauge the uniqueness of sweet and umami stimuli using an anatomical technique, such as visualization of the immediate early gene $c$-fos in PBN neurons. In fact, previous research has indicated stimulation with different taste qualities produces distinctive $c$-fos patterns in the PBN. For this current 
research study, my first hypothesis was that since previous studies suggested the similarity between sweet and umami compounds in C57BL/6J (B6) mice; stimuli of both taste qualities would produce similar levels of preference, consumption, and levels of taste reactivity behaviors. Secondly, I hypothesized that taste stimulation with either sweet (sucrose) or umami (monopotassium glutamate; MPG, or the synergistic mixture of MPG+IMP) stimuli would produce a similar $c$-fos expression pattern in sweet and umami stimuli, and this would also be distinct from the $c$-fos expression patterns elicited by both the bitter stimulus, quinine hydrochloride $(\mathrm{QHCl})$ and water.

Overall, the preference tests revealed that both sucrose and umami stimuli (especially MSG+IMP) were preferred and consumed at a similarly high level in B6 mice. However, the taste reactivity test did not yield any insight into whether the sweet and umami taste stimuli were perceived as similar. However, taste reactivity to the bitter stimulus, QHCl, was easily distinguishable from the other tested taste stimuli. Using $c$-fos immunohistochemistry to visualize neuronal activation, I then compared staining patterns of activation evoked by: water, QHCl, sucrose, saccharin, MPG, and MPG+IMP in subdivisions of the PBN in B6 mice, as well as a few other non-taste brainstem areas (locus coeruleus and mesencephalic nucleus of the trigeminal nerve). Results showed that quinine elicited significantly less $c$-fos positive nuclei in the entire dorsal lateral (DL) subnucleus compared to water. A few other significant effects of the tastant stimuli were found in the rostral portion of the waist, central lateral (CL), and DL PBN subnuclei, but distinct $c$-fos representations were not found for each stimulus tested. To determine if tastant effects might have been subtler in terms of cell density or patterning; and therefore, could have been missed using normal cell counting methods, I decided to use a three-dimensional mapping approach to examine $c$-fos expression in the PBN. Results of this new mapping approach suggest its potential usage in future studies. 


\section{TABLE OF CONTENTS}

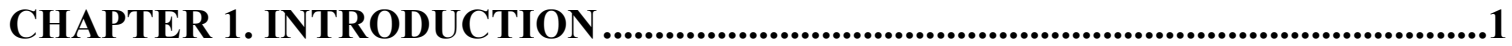

Taste.

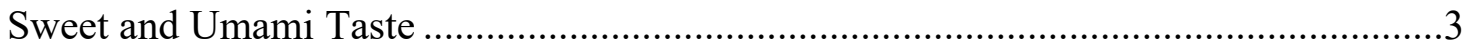

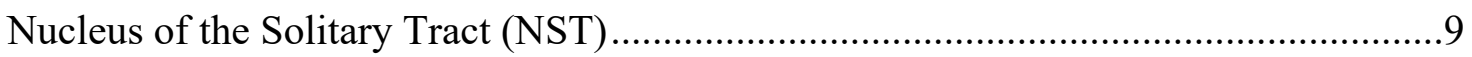

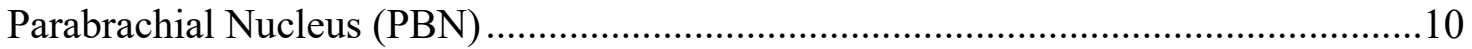

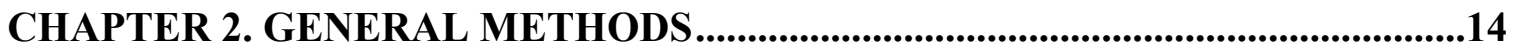

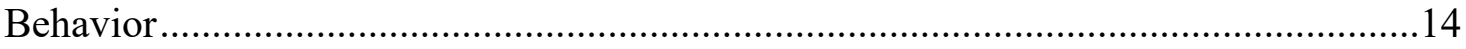

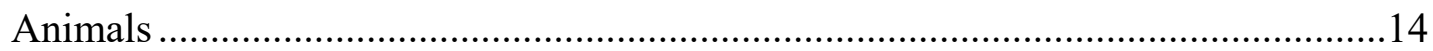

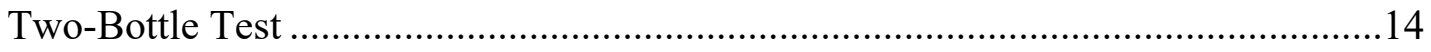

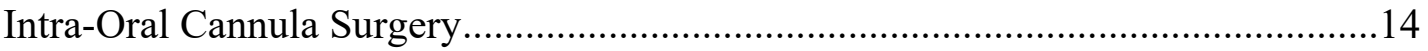

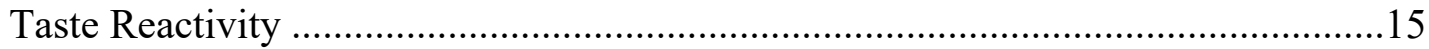

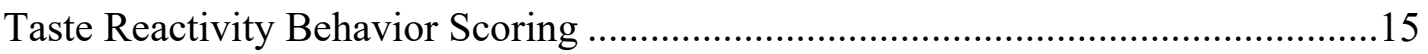

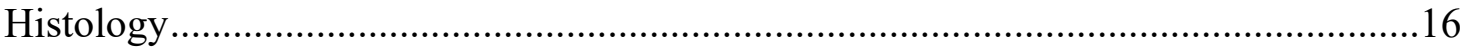

C-fos Immunohistochemistry with Nickel Intensified-3,3'Diaminobenzidine

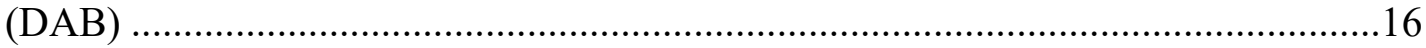

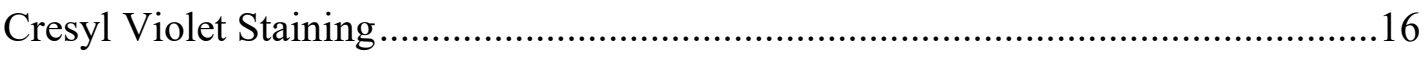

\section{CHAPTER 3. BEHAVIORAL ANALYSIS OF TASTE REACTIVITY AND TWO-BOTTLE PREFERENCE TESTS TO TASTE STIMULI IN C57BL/6J}

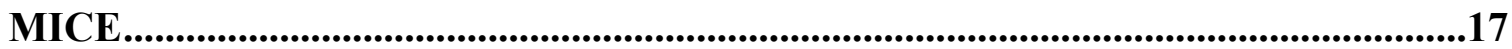

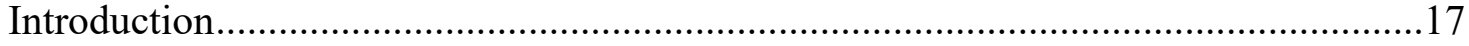

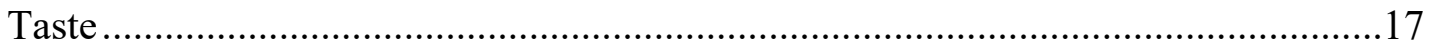

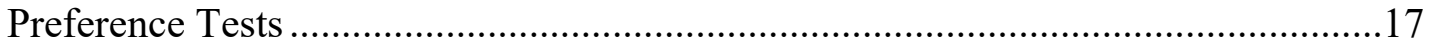

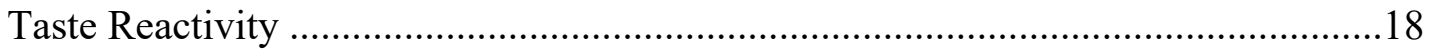

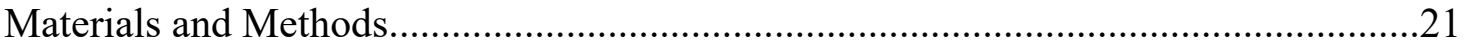

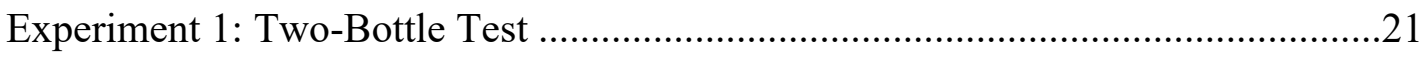

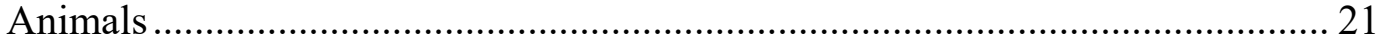

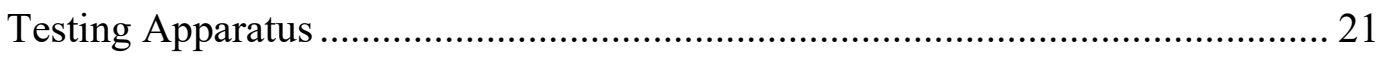

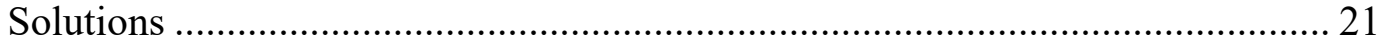

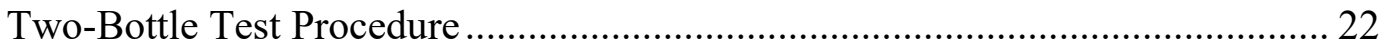

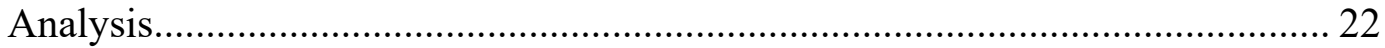

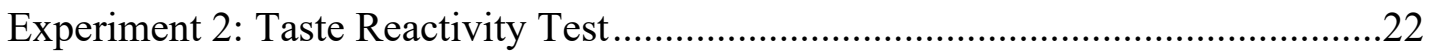

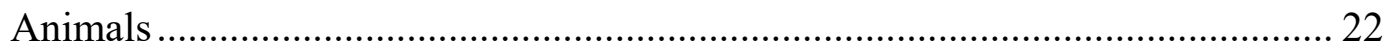

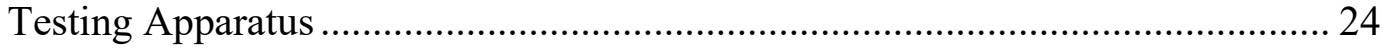

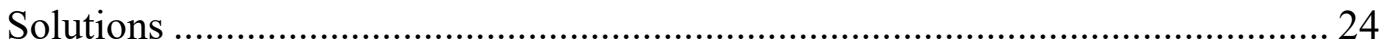

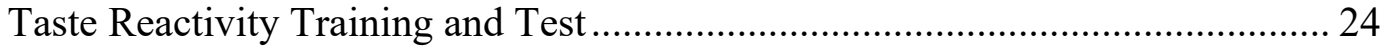

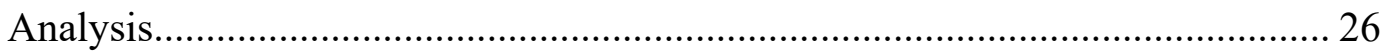

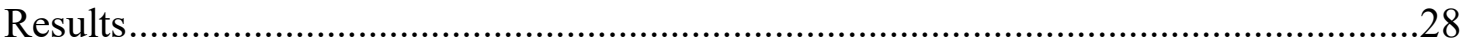

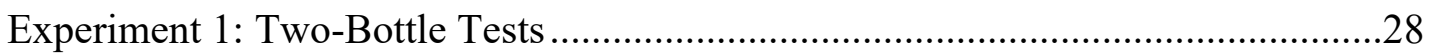




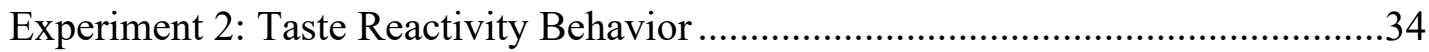

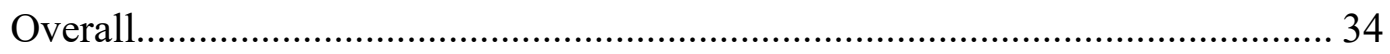

Consistency of TR Behavior Patterns during Stimulation with Each Taste

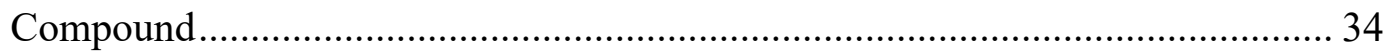

Differences in TR Behavior Between Each Stimulus.......................................... 34

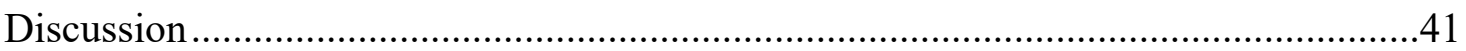

Experiment 1: Preference Tests ........................................................................41

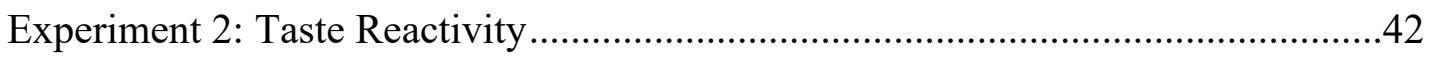

CHAPTER 4. IMMUNOHISTOCHEMICAL VISUALIZATION AND 3D MAPPING OF C-FOS ELICITED FROM SWEET, BITTER, AND UMAMI STIMULI IN TASTE PORTIONS OF THE PBN IN C57BL/6J MICE ......................47

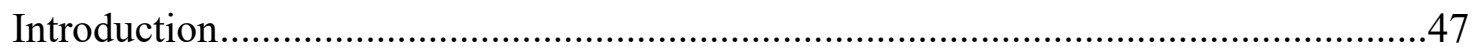

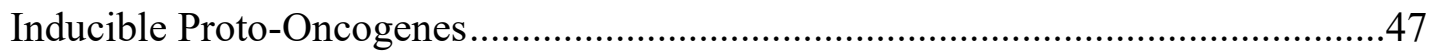

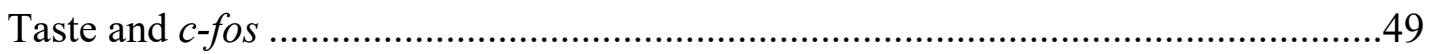

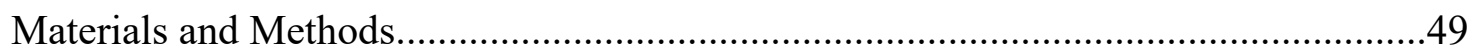

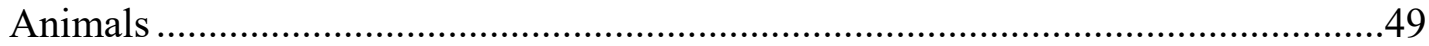

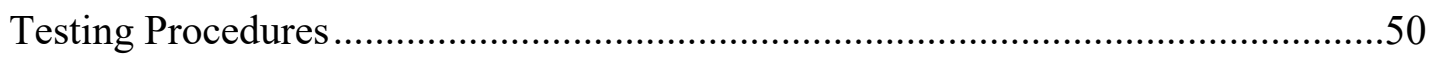

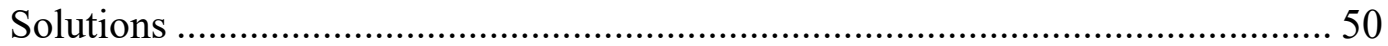

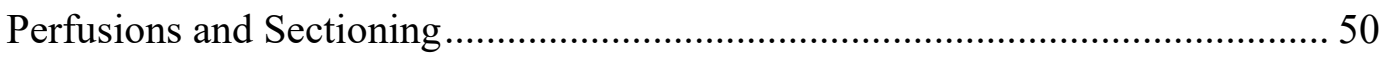

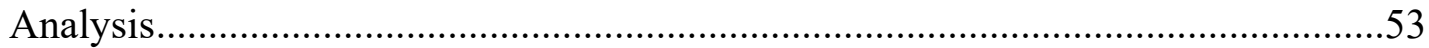

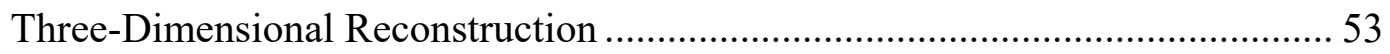

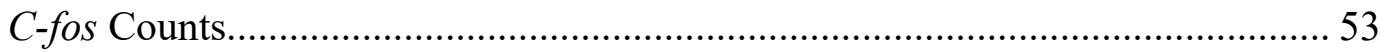

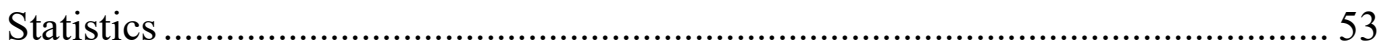

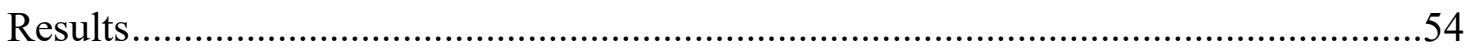

Representation of Tissue ..................................................................................54

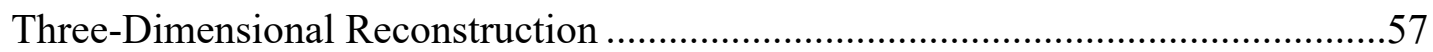

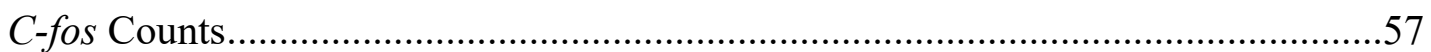

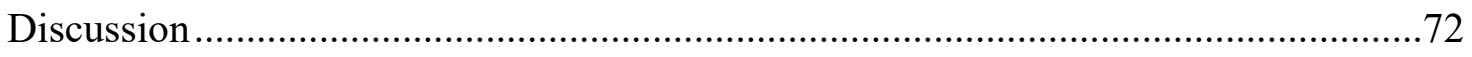

CHAPTER 5. FINAL SUMMARY AND OVERALL CONCLUSIONS .....................76

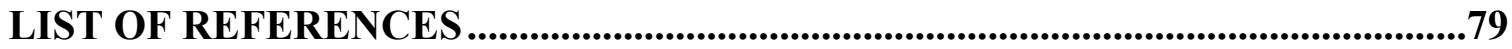

VITA 


\section{LIST OF TABLES}

Table 1-1. Classification of Each Kind of Sweetener and Where They Bind to Within the Human Taste Receptors.

Table 3-1. All Taste Stimuli Tests in One Group of 10 Animls during the TwoBottle Test.

Table 3-2. Taste Stimuli Tested, Number of Groups Tested, and Number of Animals in Each Group Used in the Taste Reactivity Test......

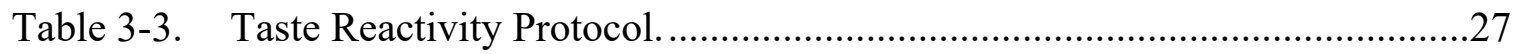

Table 4-1. Immunohistochemistry Steps Used for Labeling $c$-fos. .............................51

Table 4-2. Three-Dimensional Reconstruction Neurolucida Data...............................64 


\section{LIST OF FIGURES}

Figure 1-1. Taste Pathway in the Rodent Brain..........................................................2

Figure 3-1. Overall Comparison of Taste Solution Preference and Consumption of Sweet, Umami, and Bitter Stimuli to Water to Determine Their

Palatability in B6 Mice.

Figure 3-2. Comparison to Investigate the Similarity in Preference and Consumption

Between All of the Sweet and Umami Stimuli in B6 Mice.

Figure 3-3. Comparison to Investigate the Similarity in Preference Between

Saccharin and All of Umami Stimuli in B6 Mice.

Figure 3-4. Comparison of Preference and Consumption of the Known Bitter

Control QHCl to All Sweet and Umami Stimuli in B6 Mice.

Figure 3-5. Comparison to Investigate the Similarity in Consumption Between All of the Umami Stimuli in B6 Mice.

Figure 3-6. Non-Appetitive Taste Reactivity Behaviors in Response to All the Taste Stimuli and Their Occurrence over 20-Minute (1-Minute) Trials.

Figure 3-7. Appetitive Taste Reactivity Behaviors in Response to All the Taste Stimuli and Their Occurrence over 20-Minute (1-Minute) Trials.

Figure 3-8. Overview of Taste Reactivity Behaviors in Response to Each of the Taste Stimuli.

Figure 3-9. Taste Reactivity Behavior Responses of Individual Animals Stimulated with Water, Sucrose, and Saccharin.

Figure 3-10. The Incidence of the Occurrence of 6 Different Taste Reactivity Behaviors during Stimulation with Each of the Different Taste Stimuli.....40

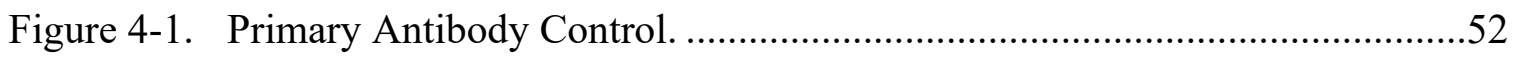

Figure 4-2. Tissue from a QHCl-stimulated Animal. ...............................................55

Figure 4-3. Diagrams of Delineated Subnuclei and Immunolabeled C-fos Created from Tissue from Animals Stimulated with DI Water, Sucrose, Saccharin, MPG, MPG+IMP, and Quinine Hydrochloride. 
Figure 4-4. Three-Dimensional Reconstruction of the Left SCP and the PBN from a

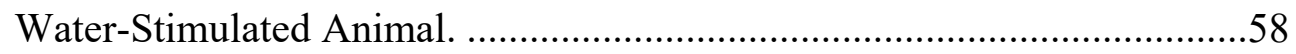

Figure 4-5. Three-Dimensional Reconstruction of the Left SCP and the PBN from a

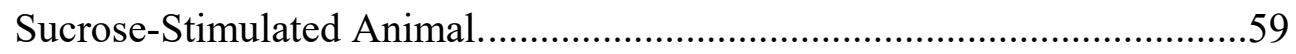

Figure 4-6. Three-Dimensional Reconstruction of the Left SCP and the PBN from a

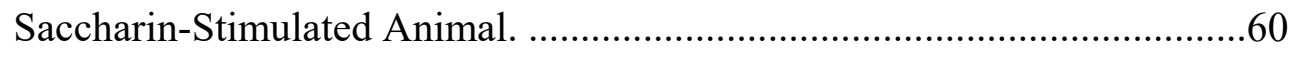

Figure 4-7. Three-Dimensional Reconstruction of the Left SCP and the PBN from

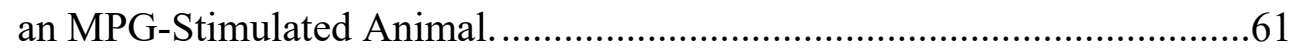

Figure 4-8. Three-Dimensional Reconstruction of the Left SCP and the PBN from a MPG+IMP-Stimulated Animal. ..............................................................62

Figure 4-9. Three-Dimensional Reconstruction of the Left SCP and the PBN from a Quinine-Stimulated Animal.

Figure 4-10. Average Number of $C$-fos Counted in the Entire Taste Portion of the PBN, LC, and Me5 for each Taste Stimulus.

Figure 4-11. Average Number of C-fos Counted in Each of the Eight PBN Subnuclei for Each Taste Stimulus.

Figure 4-12. Average Number of $C$-fos Counted in DL Subnucleus in the PBN for Each Taste Stimulus.

Figure 4-13. The Amount of $C$-fos Expression in the Caudal, Intermediate, and Rostral Levels of Each of the Eight PBN Subnuclei.

Figure 4-14. The Amount of $C$-fos Expression in the Intermediate Level DL

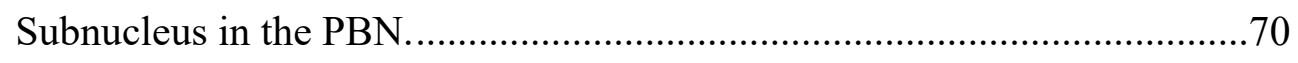

Figure 4-15. The Amount of $C$-fos Expression in the Rostral Level (A) Waist Area and (B) CL subnucleus and the (C) DL Subnucleus in the PBN. 


\section{LIST OF ABBREVIATIONS}

\begin{tabular}{|c|c|}
\hline AMP & Adenosine monophosphate \\
\hline $\mathrm{BC}$ & Brachium conjunctivum \\
\hline BST & Bed Nucleus of the Stria Terminalis \\
\hline $\mathrm{CeA}$ & Central Nucleus of the Amygdala \\
\hline CLS & Central Lateral Subnucleus \\
\hline CRD & Cysteine-rich domain \\
\hline $\mathrm{CT}$ & Chorda Tympani \\
\hline CTA & Conditioned Taste Aversion \\
\hline DAB & 3,3'Diaminobenzidine \\
\hline DI & Deionized \\
\hline DLS & Dorsal Lateral Subnucleus \\
\hline DMS & Dorsal Medial Subnucleus \\
\hline ELS & External Lateral Subnucleus \\
\hline EMS & External Medial Subnucleus \\
\hline GL & Glossopharyngeal \\
\hline GMP & Guanosine Monophosphate \\
\hline $\mathrm{HCl}$ & Hydrochloric acid \\
\hline HEK & Human Embryonic Kidney \\
\hline $\mathrm{IC}$ & Insular Cortex \\
\hline IL & Internal Lateral Subnucleus \\
\hline IMP & Inosine monophosphate \\
\hline I.P. & Intraperitoneal \\
\hline
\end{tabular}




\begin{tabular}{|c|c|}
\hline IP3R3 & Inositol 1,4,5-triphosphate receptor \\
\hline L-AP4 & $\mathrm{L}-(+)-2$-amino-4-phosphobutryic acid \\
\hline $\mathrm{LC}$ & Locus coeruleus \\
\hline LH & Lateral Hypothalamus \\
\hline $\mathrm{LiCl}$ & Lithium chloride \\
\hline MAG & Monoammonium glutamate \\
\hline Me5 & Mesencephalic nucleus of the trigeminal nerve \\
\hline MGluR4 & Metabotropic glutamate receptor 4 \\
\hline MPG & Monopotassium glutamate \\
\hline MRI & Magnetic Resonance Imaging \\
\hline MSG & Monosodium glutamate \\
\hline $\mathrm{NaCl}$ & Sodium Chloride \\
\hline $\mathrm{NH} 4 \mathrm{Cl}$ & Ammonium chloride \\
\hline NST & Nucleus of the Solitary Tract \\
\hline PAP & Peroxidase-antiperoxidase \\
\hline $\mathrm{PB}$ & Phosphate buffer \\
\hline PBN & Parabrachial nucleus \\
\hline PBS & Phosphate buffered saline \\
\hline PET & Positron emission tomography \\
\hline PLCbeta2 & 1-Phosphatidylinositol-4,5-biphosphate phosphodiesterase beta-2 \\
\hline PROP & 6-n-propylthiouracil \\
\hline QHCl & Quinine hydrochloride \\
\hline $\mathrm{Sac}$ & Saccharin preference locus \\
\hline
\end{tabular}




$\begin{array}{ll}\text { SCP } & \text { Superior Cerebellar Peduncle } \\ \text { TRC } & \text { Taste Receptor Cell } \\ \text { TRPM5 } & \text { Transient receptor potential cation channel subfamily M member 5 } \\ \text { VFT } & \text { Venus-flytrap } \\ \text { VLS } & \text { Ventrolateral subnucleus } \\ \text { VNO } & \text { Vomeronasal Organ } \\ \text { VPMPC } & \text { Medial parvocellular component of the ventrobasal complex of the } \\ \text { WGA } & \text { Thalamus } \\ \text { V } & \text { Wheat-germ agglutinin } \\ \text { VII } & \text { Trigeminal Nerve } \\ \text { X } & \text { Facial Nerve } \\ \text { XI } & \text { Vagus Nerve } \\ \end{array}$




\section{CHAPTER 1. INTRODUCTION}

\section{Taste}

The sense of taste is very important for an organism to obtain proper nutrition and as a protective mechanism. What an organism perceives a substance to taste like determines whether it will be ingested or rejected. Each of the five distinct taste qualities (sweet, sour, bitter, salty, and umami) are transduced by a distinct channel or receptor that is located on separate taste receptor cells on the tongue, soft palate, and pharynx (Smith \& Boughter, 2007). At the receptor level, three genes encoding three receptor subunits (called T1R1, T1R2, and T1R3) account for the transduction of most sweet- and umami-tasting stimuli (Li, 2009; Nelson et al, 2002; Nelson et al. 2001). Umami stimuli are recognized by a T1R1-T1R3 heterodimer, whereas sweet stimuli activate a T1R2T1R3 heterodimer or a T1R2-T1R2 homodimer (Nelson et al., 2002; Nelson et al., 2001; Roper, 2013). Bitter taste is determined, however, by a family of T2R receptors (Adler et al., 2000; Chandrashekar et al., 2000; Roper, 2013). The binding of stimuli to their corresponding sweet or umami receptor activates a G-protein-coupled receptor cascade within the taste bud (Roper, 2013; Vandenbeuch \& Kinnamon, 2009). This ultimately involves activation of a transient receptor potential cation channel subfamily $\mathrm{M}$ member 5 (TRPM5) channel, influx of calcium ions, and release of the neurotransmitter ATP onto the adjacent nerve fiber (Damak et al., 2006; Hamilton \& Norgren, 1984; Roper, 2013; Vandenbeuch \& Kinnamon, 2009).

Taste buds transmit information via branches of three cranial nerves, the facial (VII), glossopharyngeal (IX), and the vagus (X). These nerves terminate in the rostral nucleus of the solitary tract (NST) (Hamilton \& Norgren, 1984), whose neurons in turn primarily project (in rodents) to the parabrachial nucleus in the pons (PBN; Figure 1-1) (Halsell, Travers, \& Travers, 1996; Herbert, Moga, \& Saper, 1990). At this level in the brain, the gustatory pathway diverges into two major routes. The first of these is a thalamocortical pathway: PBN taste neurons synapse in the medial parvocellular component of the ventrobasal complex of the thalamus (VPMPC; Figure 1-1) (Fulwiler \& Saper, 1984; Krout \& Loewy, 2000). From the VPMpc, thalamic neurons in turn project to the gustatory area of the insular cortex (IC; Figure 1-1) (Fulwiler \& Saper, 1984; Halsell, 1992; Karimnamazi \& Travers, 1998; Lundy \& Norgren, 2004; Norgren, 1974; Norgren \& Leonard, 1973; Norgren \& Wolf, 1975; Saper \& Loewy, 1980). The secondary route of information consists of other PBN neurons that project directly to areas within the limbic forebrain, including the lateral hypothalamus (LH), central nucleus of the amygdala (CeA) and the bed nucleus of the stria terminalis (BST; Figure 1-1) (Fulwiler \& Saper, 1984; Halsell, 1992; Karimnamazi \& Travers, 1998; Norgren, 1974; Norgren \& Leonard, 1973; Norgren \& Wolf, 1975; Saper \& Loewy, 1980).

The information gathered from taste combines with olfactory information and oral somatosensory information produced via the trigeminal nerve (V) to produce what is perceived as flavor (Beauchamp \& Mennella, 2011; Small, Jones-Gotman, Zatorre, Petrides, \& Evans, 1997). A dedicated neural pathway for this phenomenon has been 


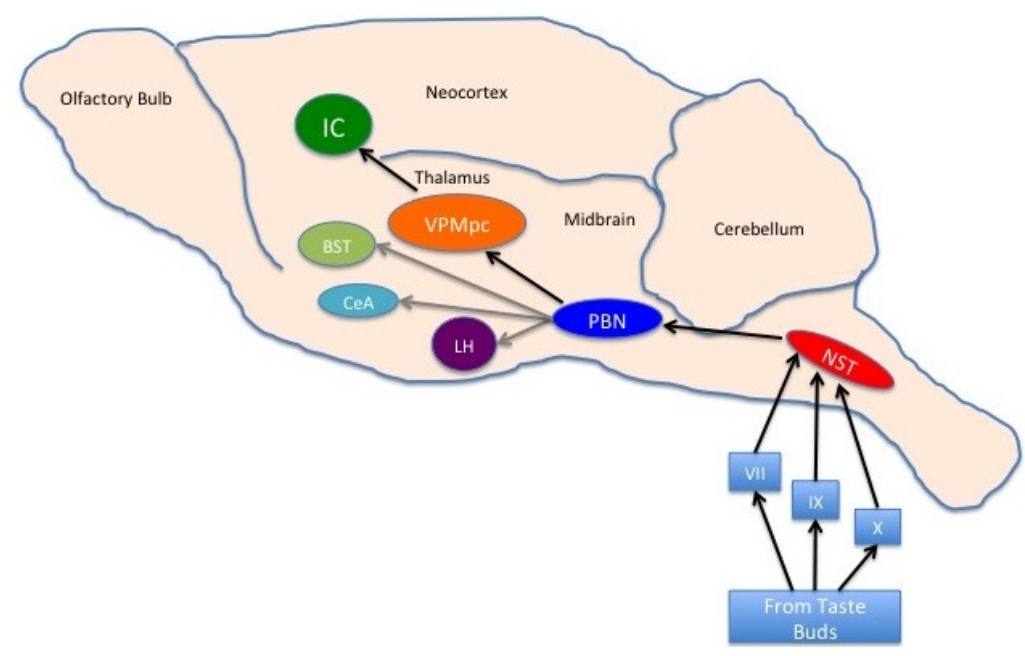

Figure 1-1. Taste Pathway in the Rodent Brain.

Notes: $\mathrm{X}=$ vagus nerve, $\mathrm{IX}=$ glossopharyngeal nerve, $\mathrm{VII}=$ facial nerve, $\mathrm{NST}=$ nucleus of the solitary tract, $\mathrm{PBN}=$ parabrachial nucleus, $\mathrm{LH}=$ lateral hypothalamus, $\mathrm{Ce} A=$ central nucleus of the amygdala, BST $=$ bed nucleus of the stria terminalis, $\mathrm{VPMpc}=$ medial parvocellular component of the ventrobasal complex of the thalamus, $\mathrm{IC}=$ insular cortex 
elucidated using Positron emission technology (PET) while subjects are smelling and tasting two compounds at the same time which either matched or were disassociated taste/smells (Small et al., 1997). These results show that the brain areas this pathway includes are: the anterior insula/frontal operculum (primary taste area) (O'Doherty, Rolls, Francis, Bowtell, \& McGlone, 2001; Rolls, 2015), the right caudolateral orbitofrontal cortex (CLOF) (secondary taste area) (O'Doherty et al., 2001; Rolls \& Baylis, 1994), a more medial location in the orbital frontal cortex (secondary olfactory cortex) (Zatorre, Jones-Gotman, Evans, \& Meyer, 1992), the left amygdaloid nucleus, and left and right basal forebrain (Small et al., 1997). While examining the increases or decreases in blood flow that occurred during these concurrent smell and taste experiments, it can be determined whether a stimulus is differentiated as a flavor or an odor within the primary gustatory cortex (Small et al., 1997). Visual information obtained from seeing food before one tastes it is also important and influences a person's eating decisions (Rolls, Rowe, \& Rolls, 1982). For example, if the color of a food is changed to something different than expected, a person may perceive it as tasting different than when it was its original color (Delwiche, 2012). Visual information seems to converge with taste and olfaction in the orbital frontal cortex (secondary taste cortex) (Rolls \& Baylis, 1994). Flavor influences taste preferences that in turn effect an organism throughout its entire life, starting in utero with exposure via amniotic fluid from the foods the mother consumes and postpartum from foods that are excreted into breast milk (Beauchamp \& Mennella, 2011). Flavor preferences directly affect diet, which is a critical factor in an organism's likelihood to develop many diseases, such as hypertension, obesity, heart disease, diabetes, and some cancers (Beauchamp \& Mennella, 2011).

\section{Sweet and Umami Taste}

A sweet taste sensation is evoked by a number of different compounds including sugars, artificial sweeteners, sugar alcohols, sweet proteins, and natural sweeteners (Table 1-1) (Nelson et al., 2001). Both humans and rodents innately and highly prefer sweet-tasting compounds. Genetic variants of T1R genes have been found to produce differences both within a single species and across species in their preference and ability to taste different sweeteners (Fernstrom et al., 2012). For example, one of the sweet taste receptors, T1R2, in the cat family is a pseudo gene and therefore non-functional, leading to a complete lack of this taste quality in cats, but leaves all the other taste qualities unaffected (Li et al., 2005). All sweet-tasting compounds bind to the same heterodimer receptor T1R2 + T1R3 (Fernstrom et al., 2012). However, different sweet stimuli have been shown to bind to different parts of the receptor (Table 1-1). The T1R portion of the sweet taste receptor contains 2 distinct portions that are attached to each other by a cysteine-rich domain (CRD): A venus-flytrap (VFT) portion located outside of the cell at the N-terminal end of the receptor and a portion composed of seven helices that spans the cell membrane at the C-terminus end of the receptor (Fernstrom et al., 2012; Vigues, Dotson, \& Munger, 2009). Thus, as a result of the differential binding of each of the different types of sweet-tasting compounds, the T1R2 + T1R3 receptor is activated, and the sweet taste quality is perceived (Fernstrom et al., 2012). 
Table 1-1. Classification of Each Kind of Sweetener and Where They Bind to Within the Human Taste Receptors.

\begin{tabular}{|c|c|c|}
\hline Sweetener & Kind & Binds to/or is needed \\
\hline $\begin{array}{l}\text { Sucrose } \\
\text { Glucose } \\
\text { Fructose }\end{array}$ & Natural sugars & $\begin{array}{l}\text { VFT domains of both T1R2 } \\
\text { and T1R3 [39] }\end{array}$ \\
\hline Sucralose & Artificial Sweetener & $\begin{array}{l}\text { VFT domains of both T1R2 } \\
\text { and T1R3 [39] }\end{array}$ \\
\hline Saccharin & Artificial Sweetener & $\begin{array}{l}\text { May bind to the cleft } \\
\text { formed by Lobe } 1 \text { and Lobe } \\
2 \text { of the ATD domain of } \\
\text { T1R2 [40] }\end{array}$ \\
\hline $\begin{array}{l}\text { Aspartame } \\
\text { Neotame }\end{array}$ & Artificial Sweetener & $\begin{array}{l}\text { A binding pocket inside the } \\
\text { 7-transmembrane spanning } \\
\text { domain of T1R2 [41] }\end{array}$ \\
\hline Sodium cyclamate & Artificial Sweetener & $\begin{array}{l}\text { Transmembrane domain in } \\
\text { T1R3 }[41,42]\end{array}$ \\
\hline $\begin{array}{l}\text { Thaumatin } \\
\text { Monellin }\end{array}$ & Sweet proteins & T1R2 receptor [43] \\
\hline Brazzein & Sweet protein & $\begin{array}{l}\text { Cysteine-rich linker in } \\
\text { T1R3 [44] }\end{array}$ \\
\hline $\begin{array}{l}\text { Erythritol } \\
\text { Sorbitol } \\
\text { Xylitol }\end{array}$ & Sugar Alcohols & Unknown \\
\hline $\begin{array}{l}\text { Neohesperidin } \\
\text { dihydrochalcone }\end{array}$ & Sweet Glycoside & $\begin{array}{l}\text { The 7-transmembrane } \\
\text { spanning domain in T1R3 } \\
\text { [45] }\end{array}$ \\
\hline $\begin{array}{l}\text { Steviol } \\
\text { Rebaudioside A }\end{array}$ & Sweet Glycoside & Unknown \\
\hline Acesulfame potassium & Artificial Sweetener & $\begin{array}{l}\text { May bind to the cleft } \\
\text { formed by Lobe } 1 \text { and Lobe } \\
2 \text { of the ATD domain of } \\
\text { T1R2 [40] }\end{array}$ \\
\hline
\end{tabular}


Umami taste, while proposed by Ikeda in 1909, is the most recently accepted of the five basic taste qualities and therefore has been the least characterized (Ikeda, 1909, 2002). Umami means 'delicious taste' (also described as a pleasant savory taste) in Japanese (Yamamoto, Matsuo, Kiyomitsu, \& Kitamura, 1988). There are many compounds that elicit umami taste, with the most recognized being glutamic acid (monosodium glutamate or MSG) (Birch, 1987; Kawai, Okiyama, \& Ueda, 2002), but also some other L-type amino acids such as monopotassium glutamate (MPG) and Laspartate (Kawai et al., 2002), as well as certain ribonucleotides, including monoammonium glutamate (MAG) (Inoue, Beauchamp, \& Bachmanov, 2004), inosine monophosphate (IMP) (Bachmanov, 2010; Inoue et al., 2004; Kawai et al., 2002), guanosine monophosphate (GMP) (Bachmanov, 2010; Inoue et al., 2004; Kawai et al., 2002), adenosine monophosphate (AMP) (Bachmanov, 2010; Keast, Canty, \& Breslin, 2004), along with other compounds including ibotenic acid (Bachmanov, 2010; Kawai et al., 2002), tricholomic acid (A. Bachmanov, 2010), theonine (Bachmanov, 2010; Narukawa, Toda, Nakagita, Hayashi, \& Misaka, 2014), and theogallin (Bachmanov, 2010). One important characteristic of umami taste is the occurrence of "synergism," in which a mixture of glutamate with a ribonucleotide, such as inosine monophosphate (IMP), produces a response considerably stronger than predicted by the responses to the individual components (Yamaguchi, 1991). Umami stimuli are recognized in taste cells by a T1R1-T1R3 heterodimer (Li et al., 2002; Nelson et al., 2002), although there is also some evidence for additional receptors, including a truncated taste-specific metabotropic glutamate receptor (mGluR4) (Chaudhari et al., 1996; Li et al., 2002; Nelson et al., 2002; Toyono et al., 2002), a truncated taste-specific mGluR1 (San Gabriel, Uneyama, Yoshie, $\&$ Torii, 2005), as well as two brain-expressed metabotropic glutamate receptors, brainmGluR1 (San Gabriel et al., 2005) and brain-mGluR4 (Toyono et al., 2002). These receptors may all contribute to umami taste, but each may contribute differently (Nakashima, Eddy, Katsukawa, Delay, \& Ninomiya, 2012). T2R1+T1R3, taste-mGluR1, and taste-mGluR4 receptors have a lower binding affinity for glutamate compared to brain-mGluR1 and brain-mGluR4 (Chaudhari, Landin, \& Roper, 2000; Chaudhari et al., 1996; Lindemann, 2000; Nakashima et al., 2012). In the mouse, the umami T1R1/T1R3 receptor is found in the taste papillae located on the anterior tongue, posterior tongue, and the palate (Li et al., 2002; Nelson et al., 2002). This umami heterodimer receptor responds to many L-type amino acids as observed in electrophysiological experiments (Nelson et al., 2002). The human counterpart of the T1R1/T1R3 umami receptor, to date, has been successfully activated by L-glutamate (Li et al., 2002; Nelson et al., 2002). It has also been discovered that $\mathrm{L}$-glutamate does not produce a response from cells containing only one of the human T1R1 or T1R3 receptors (Li et al., 2002). Brain- and taste-mGluR4 receptors and a taste-mGluR1 are located in taste cells on the rat tongue in all three types of taste papillae (fungiform, foliate, and circumvallate) (Chaudhari et al., 1996; Toyono et al., 2002; Toyono et al., 2003). Also in rats, the variant mGluR1, containing a truncated $\mathrm{N}$-terminal domain, has been isolated in circumvallate and foliate papillae on the posterior portion of the tongue (San Gabriel et al., 2005; Yasumatsu et al., 2009).

In mice, differences in sweetener preference have been found between different strains of mice. These differences are produced by different versions of the saccharin 
preference (Sac) locus, which has been mapped to distal chromosome 4 (Bachmanov, $\mathrm{Li}$, et al., 2001; Blizard, Kotlus, \& Frank, 1999; Fuller, 1974; Lush, 1989). The Sac locus, on chromosome 4, is located either at or very near to the T1R3 receptor (Bachmanov, Li, et al., 2001; Montmayeur, Liberles, Matsunami, \& Buck, 2001; Nelson et al., 2001; Sainz, Korley, Battey, \& Sullivan, 2001), which is shared by both sweet and umami taste receptors (T1R3). The Sac locus determines whether a mouse strain is a taster (i.e. C57BL/6J (B6) mice) or a non-taster (i.e. DBA/2J (D2) mice) of sweet stimuli (Capeless \& Whitney, 1995; Montmayeur et al., 2001). In a study that examined both the nucleotide and amino acid sequence of T1R3 in taster and non-taster strains, ultimately only two nucleotide sequences were found to differ between them. They were located at T55A (positron 55, where the tasters had a T encoded and non-tasters had an A) and at I60T (positron 60, where tasters had an I encoded and non-tasters had a T) (Max et al., 2001). The amino acid substitution that occurs at I60T may introduce a potential glycosylation site that would prevent the receptor from functioning by rendering it unable to form dimers (Max et al., 2001). Mice that exhibit as tasters are approximately 5 times more perceptive to sweet compounds than mice that are non-tasters (Nelson et al., 2002). This has been illustrated by behavioral studies, in which taster strains of mice consume and prefer larger amounts of sweet compounds compared to non-taster strains (Bachmanov, Tordoff, \& Beauchamp, 2001; Capeless \& Whitney, 1995; Lush, 1989), and by an electrophysiological study, in which the level of chorda tympani activation in response to sweet stimuli corresponded to whether a strain was a taster or non-taster (Inoue, McCaughey, Bachmanov, \& Beauchamp, 2001). The analogous portion of the genome in humans to the Sac locus has been found to be at chromosome 1p35 and 1p36 (Bachmanov et al., 1997; Montmayeur et al., 2001). This portion contains the taste receptor, hT1R3, which is homologous to the mT1R3 (Montmayeur et al., 2001). In humans, in regard to sweet preference, there has not been a similar phenomenon found as in mice, where a difference in genotypes has been found, but one study did identify a quantitative trait loci on chromosome 16 that was linked to how often sweet foods are consumed, indicating some genetic variability may exist (Keskitalo et al., 2007).

Sweet and umami tastants provoke distinct perceptions in adult humans, with umami stimuli often characterized as having a savory, or perhaps "brothy" taste (Mouritsen, 2013). Even though one study has found that these two taste qualities provoke similar but distinct perceptions in humans, (Steiner, 1987) other studies performed in both humans (Cairncross \& Sjostrom, 1948) and in rodents (Nishijo, Ono, \& Norgren, 1991), have found conflicting results, which makes the evidence for the distinctness of sweet and umami sensations less clear. The taste of the umami compound MSG is indeed multifaceted and seems to consist of a salty component (thought to be contributed to by the sodium cation it contains) and a sweet component (thought to be contributed to by the glutamic anion) (Nishijo et al., 1991). Behavioral conditioned taste aversion (CTA) studies in rats and behavioral discrimination studies in mice have provided evidence that may classify MSG as a combination of salty and sweet taste (if amiloride was not added to the solution) and not as a completely separate taste quality (Breza, Curtis, \& Contreras, 2007; Delay, Hernandez, Bromley, \& Margolskee, 2006; Ruiz, Wray, Delay, Margolskee, \& Kinnamon, 2003; Yamamoto et al., 1991; Yamamoto, Yuyama, Kato, \& Kawamura, 1985b). One the other hand, a recent CTA study performed 
in mice has found that MPG+IMP generalized to sucrose (Saites, Goldsmith, Densky, Guedes, \& Boughter, 2015) and an older study found that MSG+IMP with the addition of amiloride also generalized to sucrose (Yamamoto et al., 1991) as opposed to umami stimuli. Another behavioral discrimination study in rats also observed that the addition of amiloride makes it more difficult to discriminate between sucrose and MSG (Heyer, Taylor-Burds, Mitzelfelt, \& Delay, 2004). Both sweet and umami stimuli are found in many foods and are characterized as highly preferred by many animals (Ohara \& Naim, 1977; Waldern \& van Dyk, 1971; Zhang et al., 2003).

Electrophysiological data in mice suggest that the immediate neuronal activation generated from both sucrose and the synergistic mixture of the umami stimulus, MPG+IMP, is highly correlated across the entire population of PBN taste neurons (Tokita, Yamamoto, \& Boughter, 2012). A similar phenomenon has been found in rats when PBN taste neurons are stimulated with either sucrose and MSG+GMP (Nishijo et al., 1991). Conversely, with a lower concentration of MPG+IMP, the activity of PBN neurons in response to MPG+IMP or sucrose were found to be less similar (Geran \& Travers, 2009). Within the PBN, many neurons have been observed to respond to both MSG and sucrose (Nishijo et al., 1991). Thus, sweet and umami taste share many common factors in rodents, especially in terms of transduction mechanism and central representation within the PBN.

Two types of genetic manipulation have been used to investigate taste pathways, knockout and transgenic mice. Knockout mice have been created for most of the critical proteins in the taste transduction cascade. Both inositol 1,4,5-triphosphate receptor (IP3R3) (Hisatsune et al., 2007) and alpha-gustducin (Danilova, Damak, Margolskee, \& Hellekant, 2006; He et al., 2004; Ruiz et al., 2003) knockouts show decreased preference and smaller electrophysiological responses to sweet and umami. TRPM5 knockouts show either a complete loss (Zhang et al., 2003) or abnormal sweet and umami taste responses (Damak et al., 2006). 1-Phosphatidylinositol-4,5-biphosphate phosphodiesterase beta-2 (PLCbeta2) knockouts show a complete loss of sweet and umami taste (Zhang et al., 2003). T1R3 knockouts display a complete lack of preference for artificial sweeteners and reduced preference and electrophysiological responses to sugars and umami compounds (Damak et al., 2003). Transgenic mice have been created to map the neuronal circuitry of a portion of sweet and umami taste by expressing both wheat-germ agglutinin (WGA) in combination with the T1R3 taste receptor and have been used to map the neuronal circuitry of a portion of sweet and umami taste (Sugita \& Shiba, 2005). These mouse studies demonstrate both a definite biochemical and neuronal synaptic link between sweet and umami taste.

On the contrary, animal studies in rats using electrophysiology (Yamamoto, Yuyama, Kato, \& Kawamura, 1985a; Yamamoto et al., 1985b) and in vivo optical imaging techniques (Accolla, Bathellier, Petersen, \& Carleton, 2007; Yoshimura, Sugai, Fukuda, Segami, \& Onoda, 2004) found that each taste (sweet, sour, salty, and bitter) activated a distinct and unique configuration of neurons within the gustatory cortex that respond best to each of these four taste qualities in the gustatory pathway. In humans, it has been found via magnetic resonance imaging (MRI) that there is a distinct area for 
each basic taste, with some overlap, in the different areas in the gustatory cortex when each is consumed (Schoenfeld et al., 2004). However, none of the animal studies have investigated whether umami compounds activate a distinct set of CNS neurons or utilize portions that are characterized as belonging to the sweet pathway.

There are a few pharmacological compounds that interact with the taste receptors on the tongue and they have been used to aid in the investigation of sweet and umami taste, in conjunction with electrophysiological techniques in rodents. Pronase E, a compound that inhibits the response to sweet taste entirely, also partially inhibits the neuronal activity from the chorda tympani (CT; VII cranial nerve innervating anterior tongue taste buds) when exposed to MSG and a synergistic mixture of MSG+IMP (Nakashima, Katsukawa, Sasamoto, \& Ninomiya, 2001). Another compound, gurmarin, a known sweet taste inhibitor in rodents, almost entirely inhibits the response of the CT nerve to the synergistic mixture of MSG+IMP in rats (Nakashima et al., 2001). These studies suggest that umami compounds (MSG and MSG+IMP) may bind to sweet taste receptors that are innervated by the CT nerve in rats (Nakashima et al., 2001). These inhibitors also have provided results in mice, that demonstrate there may be two different pathways that can be activated by umami compounds. Activation can occur either through what is thought to be the typical umami pathway, innervated by the glossopharyngeal (GL; innervates anterior tongue taste buds) nerve or an alternate gurmarin-responsive pathway utilizing sweet taste receptors that are innervated by the CT (Nakashima et al., 2001).

Many behavioral tests exhibit similarities between the taste of sweet and umami compounds. In infants, the gustofacial reflex (Steiner, 1987) elicited during taste reactivity tests of both sweet and umami stimuli are similar, consisting of lip licking, smacking, and sucking (Steiner, 1987). In two-bottle preference tests, mice prefer and consume similar amounts of both sweet and umami compounds (Bachmanov, Tordoff, \& Beauchamp, 2000). In some behavioral experiments performed in rodents using MSG as a prototypical umami stimulus, it is clear that animals can discriminate MSG from a sweet stimulus based at least in part on the sodium ion in MSG, which is often in a sufficient concentration so as to impart a salty side taste to this stimulus. In experiments using a CTA paradigm, when MSG mixed with amiloride (an epithelial sodium channel blocker) is used as the conditioned stimulus, it was found that this was transferred to because it was perceived as similar (generalized) and also produced avoidance to sucrose (Stapleton, Roper, \& Delay, 1999; Yamamoto et al., 1991), glucose, saccharin, the artificial sweetener SC-45647, as well as produced slight avoidance to maltose in rats (Heyer et al., 2004; Spector, Breslin, \& Grill, 1988). MPG+IMP has also been found to produce avoidance of sucrose in mice (Saites et al., 2015). This shows that this synergistic mixture with amiloride is perceived to have a similar enough taste to sweet stimuli that it is not consumed because the rodent believes it will make it sick. In operant taste discrimination tests, rats could discriminate between MSG and sucrose solutions if they were greater than $50 \mathrm{mM}$ and if amiloride was added to MSG (Stapleton, Luellig, Roper, \& Delay, 2002). In the same study, without amiloride, rats could discriminate between MSG and sucrose at concentrations as low as $10 \mathrm{mM}$ (Stapleton, Luellig, Roper, \& Delay, 2002). In another study, if amiloride was not included, rats taught to 
discriminate between the four basic taste qualities generalized the taste of MSG to sucrose and also to sodium chloride $(\mathrm{NaCl})$ (Grobe \& Spector, 2008).

In contrast, in other taste discrimination studies, rats could easily tell the difference between MSG, glucose, and artificial sweeteners: saccharin and SC45647 (Heyer et al., 2004). When amiloride was added to MSG, rats could still discriminate between MSG and sucrose and MSG and SC45647, but had a more difficult time (Heyer et al., 2004). Only one taste discrimination study has been performed in mice. In this study, B6 and T1R3 knockout mice were able to discriminate between sucrose and MSG, and sucrose and MSG+amiloride, but the correct choices did drop slightly with the latter condition (Delay et al., 2006). In a CTA study with B6 mice, an aversion to $0.1 \mathrm{M}$ sucrose generalized to and produced avoidance of the synergistic mixture of MSG+IMP (Nakashima et al., 2001). This study also found that this generalization was lost when gurmarin (a known sweet-taste inhibitor) was added to the solution and returned when beta-cyclodextrin (an inhibitor of gurmarin) was subsequently added (Nakashima et al., 2001). As illustrated by the number of behavioral similarities and differences between these two primary taste qualities sweet and umami, I decided to investigate these two taste qualities using taste reactivity and examining the elicited behavioral responses in B6 mice.

\section{Nucleus of the Solitary Tract (NST)}

The nucleus of the solitary tract (NST) is an important autonomic brain area located in the medulla, which receives and relays taste information (King, 2007). In this area, taste and visceral sensory information are partitioned into the different levels along the rostral-caudal axis (Saper, 2004). In rodents, the facial nerve terminates in the most rostral part of the NST, followed by the glossopharyngeal nerve more caudally, and lastly, the vagus nerve synapses most caudally (Lundy \& Norgren, 2004). This taste portion of the NST encompasses the area "from the rostral tip caudally to the point at which the medial border of the nucleus abuts the fourth ventricle, a distance of about 2.0 mm" (Lundy \& Norgren, 2004, pg. 895). Taste information represented in the rostral portion of the NST is likely modulated before it is relayed to the PBN (Di Lorenzo, Platt, \& Victor, 2009; Lundy \& Norgren, 2004). The projection to the PBN from the NST is the largest of its outputs, with other projections terminating in: the medullary reticular formation, periaqueductal grey matter, CeA, BST, the median preoptic, paraventricular and dorsomedial hypothalamic nuclei, the lateral hypothalamic area, the dorsal motor vagal nucleus, the nucleus ambiguous, and the superior and inferior salivatory nuclei (Lundy \& Norgren, 2004; Saper, 2004). In primates, however, it has been found that even though there is a synapse between both the caudal and medial portions of NST and the PBN, taste information instead ascends directly from the rostral NST to the thalamus (Beckstead, Morse, \& Norgren, 1980). Also, in primates, the NST does not project directly to the hypoglossal, facial, or trigeminal motor nuclei, which are involved in feeding reflexes in the rat (Beckstead et al., 1980). 


\section{Parabrachial Nucleus (PBN)}

The rodent parabrachial nucleus is located in the pons and has been observed via electrophysiology to be involved in: taste (Halsell \& Travers, 1997), urination (Lumb \& Morrison, 1987), thermal nociception (Menendez, Bester, Besson, \& Bernard, 1996), salt and thirst regulation (Roncari et al., 2014), as well as the cardiovascular (Dampney, 1994), respiratory (Dawid Milner et al., 2003), and gastrointestinal systems (Baird, Travers, \& Travers, 2001). The cells located in this nucleus are relatively small (approximately 8-24 micrometers) and are hard to distinguish from each other when stained with cresyl violet (Hashimoto, Obata, \& Ogawa, 2009; Lundy \& Norgren, 2004). The PBN is a crucial component within the autonomic nervous system and ties together "medullary reflex control and forebrain behavioral and integrative regulation of the autonomic system” (Saper, 2004, pg. 768).

The mouse PBN consists of many subnuclei, including the waist area, dorsal medial (DMS), external medial (EMS), central lateral (CLS), internal lateral (ILS), ventral lateral (VLS), dorsolateral (DLS), and the external lateral (ELS) (Hashimoto et al., 2009). The waist area consists of the cells in the "narrow region surrounding the [superior cerebellar peduncle] SCP" and contains three different types of cells- "round and flat cells parallel to the SCP as well as the internal bridge cells," (Hashimoto et al., 2009 , pg. 4). The DMS is located "capping medial dorsal tip of the SCP" and contains "intensely stained medium cells," (Hashimoto et al., 2009, pg. 4). The EMS is located in the "caudal 2/3 of the PBN, capping the lateral tip of the SCP" and contains both "small oval or fusiform cells" and "large multipolar neurons" (Fulwiler \& Saper, 1984; Hashimoto et al., 2009, pg. 4). The ILS is a "cluster of large intensely stained cells" (Hashimoto et al., 2009, pg. 4). The VLS contains "lightly stained, small fusiform cells, covering the dorsolateral boundary of the SCP including the dorsal part of the waist area" (Hashimoto et al., 2009, pg. 4). The DLS consists of "intensely stained medium cells separated from the SCP by the CLS" (Hashimoto et al., 2009, pg. 4). The ELS contains two layers (an outer visceral layer of large cells and an inner taste layer of smaller cells), and although these layers can be differentiated in rats, it is difficult to determine in mice (Hashimoto et al., 2009).

The PBN receives synaptic input from many areas of the brain (Tokita, Inoue, \& Boughter, 2009). The gustatory portion of the PBN receives taste and visceral information from the rostral NST and in turn integrates and relays this information to the rest of the taste pathway. Evolutionarily, this function of the PBN may be considered to be rudimentary in rodents, since the characterized functions of the PBN seem to have been integrated into the NST within primates (Beckstead et al., 1980; Di Lorenzo et al., 2009). Taste in the PBN has been widely studied in the rat, but not in the mouse.

Recently, the mouse has become an important model to use in studying the gustatory system, because of the various genetic manipulations that can be utilized via knockout and transgenic mice (Tokita et al., 2009). 
In rodents, the PBN is an important area in the brain for both the relay of taste and visceral information, but there seems to be some debate as to whether the separation of these two different types of information is preserved, or if it is integrated in neurons within this nucleus. Electrophysiological studies have shown that taste neurons are found in portions of the medial subnucleus and VLS near the brachium conjunctivum (BC), in the BC, and in the external medial and external lateral subnuclei in the PBN (Halsell \& Frank, 1991; Halsell \& Travers, 1997; Norgren \& Pfaffmann, 1975; Tokita \& Boughter, 2016). Neurons involved in gastrointestinal functions (Baird et al., 2001) are found predominantly in the ELS, and neurons involved in respiratory functions are found in the medial and lateral subnuclei (Dawid Milner et al., 2003). Cardiovascular functions have been located, via anatomical fos-mapping studies, in the CLS and ELS (Hayward \& Castellanos, 2003). There is also anatomical evidence demonstrating segregation of the taste and visceral information that is received from the different regional projections that go between the NST and the PBN (Herbert et al., 1990).

Conversely, there are some electrophysiological studies showing that there is possible integration of visceral and taste stimuli, with each study using different kinds of visceral stimulation. One investigation used electrophysiology to record from a population of PBN neurons (approximately half of their sample size) that responded to both sodium chloride $(\mathrm{NaCl})$ and electrical vagal stimulation (Hermann \& Rogers, 1985). A different study, performed by Hajnal et al, infused a lipid solution, Intralipid, into the gut and then recorded electrophysiologically from individual PBN neurons in response to intra-oral infusions, on the tongue of awake and behaving rats, in response to the following solutions: sucrose, $\mathrm{NaCl}$, citric acid, and quinine hydrochloride $(\mathrm{QHCl})$ (Hajnal, Takenouchi, \& Norgren, 1999). They observed that quickly following the ingestion of fat, taste responses to sucrose and $\mathrm{NaCl}$, within the neurons of the PBN, were significantly reduced, showing gastrointestinal feedback occurring and interacting with the taste system (Hajnal et al., 1999). A more recent study, performed by Baird et al., also observed visceral and taste integration, and used gastric distension along with and without application of several different taste stimuli onto the tongue including: QHCl, $\mathrm{NaCl}$, sucrose, citric acid, and ammonium chloride (NH4Cl) (Baird et al., 2001). Both Hajnal et al. and Baird et al. showed that this visceral and taste integration occurs; because the magnitude of the responses elicited from the different types of taste stimuli was directly affected by both of the two different types of visceral stimulation (Baird et al., 2001; Hajnal et al., 1999). Lastly, behavioral studies utilizing CTA in combination with lesions of the PBN demonstrated the integrative function of the PBN between the sensory aspects (taste, conditioned stimulus) and visceral aspects of consuming substances (unconditioned stimulus) that are needed for an animal to create associations. based on whether a food item should be consumed or is poisonous (toxic) and should not be (Grigson, Reilly, Shimura, \& Norgren, 1998).

In order to discover some of the functions of the PBN, scientists have lesioned both the PBN and the pathways leading from it to see what effects the lack of input produces, including on the dopamine system (reward pathway) and on CTA. Excitotoxic lesions of the PBN (but not of the thalamus) prevent the release of dopamine in the nucleus accumbens, a nucleus in the reward pathway, in response to the consumption of 
sucrose (Hajnal \& Norgren, 2005). Also, lesions of the pathway from the PBN to the limbic system reduce the amount of dopamine produced in response to consuming hedonic substances, such as sucrose (de Araujo, 2009). When the medial portion of the PBN is lesioned in rats, the entire set of instinctual behaviors in CTA is obliterated and they are unable to create, keep, or remember a CTA to a taste stimulus (de Araujo, 2009). When both sides of all but the farthest lateral portions of the PBN lateral and medial subnuclei are lesioned in rats, they are also unable to form a CTA to a particular taste stimulus, but are still able to form a weak CTA, because they retain the ability to recognize flavor when it is used as a conditioned stimulus (Sclafani, Azzara, Touzani, Grigson, \& Norgren, 2001).

To try to elucidate how taste is represented in the PBN, scientists have performed studies using both in vivo electrophysiology and neuroanatomical studies with the neuronal activity marker $c$-fos. Yamamoto and co-workers have performed several investigations using a variety of taste compounds on rats, and subsequently performing immunochemistry in the PBN for the early gene $c$-fos protein. These studies have revealed that neurons located in PBN subnuclei are activated by many compounds and functions, such as: salt taste (Yamamoto, Shimura, Sakai, \& Ozaki, 1994; Yamamoto et al., 1993; Yamamoto et al., 2009), consumption of preferred and familiar substances (Yamamoto et al., 1994; Yamamoto et al., 2009), activation by aversive compounds, such as quinine (Travers, Urbanek, \& Grill, 1999; Yamamoto et al., 1994; Yamamoto et al., 1993), and gastrointestinal visceral stimuli (Yamamoto et al., 1994). Anatomical and electrophysiological evidence has suggested that the medial PBN is where the taste neurons are primarily located and are involved in palatable taste information (de Araujo, 2009; Yamamoto et al., 1994). The lateral PBN tends to be where aversive taste information and visceral information (along with cardiovascular, respiratory, and other gastrointestinal information) is primarily found (de Araujo, 2009; Yamamoto et al., 1994). These studies have been primarily done in rats, but not in mice.

Due to evidence that suggested sweet and umami stimuli taste similar to rodents, along with the fact that mice have not been investigated as thoroughly as rats have, B6 mice (a sweet preferring strain) were chosen to investigate sweet and umami taste using both two-bottle tests and taste reactivity. It was expected that sweet and umami stimuli would be preferred and consumed at the same levels and would elicit similar levels of the different taste reactivity behaviors. In the first experiment, a two-bottle test was given to measure the consumption of a battery of taste stimuli (including two concentrations of sweet stimuli and two concentrations of five different umami stimuli) and allowed the calculations of both the mean total solution consumed and the mean preference ratio for each of the taste stimuli tested. In a second experiment, the taste reactivity behavioral test was performed and allowed for the quantification of nine behaviors, some of which were appetitive and others that were not, produced in response to each of the six taste stimuli used. After the conclusion of this test, the immunohistochemical method, $c$-fos was used to see activated neurons in response to each of the six taste stimuli. The pattern of $c$-fos positive neurons was then investigated in the PBN. This area in the gustatory pathway was chosen because it is a critical relay in the gustatory pathway, along with the fact that the $c$-fos expression pattern elicited from umami compounds in this brain region of mice 
had not yet been elucidated. The distribution of activated PBN neurons in response to umami and other tastants was determined using two-dimensional analysis of mouse brain sections and by creating a three-dimensional representation of the PBN as a new way of interpreting their location. It was expected that while the different taste stimuli consumed would produce distinct patterns of activation of neurons within the subnuclei of PBN, the sweet and umami compounds would produce similar patterns.

Therefore, the first specific aim of these studies is to test the hypothesis that sweet and umami compounds (including synergistic mixtures) would both produce a similar level of preference, consumption, taste reactivity behavior occurrences, and kinds of taste reactivity behaviors. Furthermore, the second specific aim of these studies is to create a neuronal map of $c$-fos positive nuclei in response to different taste stimuli to test the hypothesis that sweet and umami taste compounds (specifically sucrose and the synergistic MPG+IMP+amil mixture) will elicit similar patterns of $c$-fos positive neurons in the PBN of B6 mice while the other taste stimuli would produce distinct patterns of $c$ fos positive nuclei based on taste stimulus. 


\title{
CHAPTER 2. GENERAL METHODS
}

\author{
Behavior
}

\begin{abstract}
Animals
All animals used were either male or female C57BL/6J (B6) mice. The B6 mice were bred from mice purchased from Jackson Laboratory (Bar Harbor, ME). The mice used were between the ages of 3-5 months old, were group housed in standard mouse cages $(28 \times 17.5 \times 13 \mathrm{~cm})$, were kept in a temperature- and humidity-controlled colony room on a 12-h light/dark cycle, and were given free access to normal dry pellet chow (22/5 rodent diet, Harlan Teklad, Madison, WI, USA) and water. See detailed Methods and Materials portion of Chapters $\mathbf{3}$ and $\mathbf{4}$ for specific information on group numbers and food and water for each set of experiments.
\end{abstract}

\section{Two-Bottle Test}

Mice were tested in individual plastic mouse cages with wire mesh grids and kept in the animal facility during the entire test. Two Pyrex glass test tubes with metal sipper tubes inserted inside rubber stoppers (bottles) were put side by side into spaces between the metal grid on the right side where food is normally placed and the rodent chow was placed where the normal water bottle would be. The metal sipper tubes of both bottles were placed exactly the same distance into the cage from the mesh grid. The amount of each liquid consumed was weighed every 24-hours (during the light cycle) and solutions were refilled as needed. The bottles w/ sipper tubes were then put back into the cage as described previously except that the bottle positions were switched to prevent a possible side bias (Bachmanov, Reed, Beauchamp, \& Tordoff, 2002; Lush, 1984; Tordoff \& Bachmanov, 2002). The body weight of each mouse was also measured every 48-hours. Each mouse was housed individually and given ad libitum access to either solution for 48-hours and then the bottles were removed, cleaned, and refilled with a new solution.

\section{Intra-Oral Cannula Surgery}

Each mouse was weighed and then anesthetized intraperitoneally (i.p.) with ketamine/xylazine (100/10 ml/kg from Butler Schein Animal Health, Dublin, OH, USA) and the hair, skin, and tissue on the top of the skull was removed. The animal was then head-fixed in a stereotaxic frame. A small hole was drilled into the right side of the animal's skull and a small screw was inserted into the hole and tightened to become an anchor. The mouse was then removed from the stereotaxic frame. One end of the cannula tubing (P50) was melted and flattened by heat and the opposite end had a slightly bent 19-gauge needle inserted into it. The needle (inside the tubing) was then inserted into the mouth just behind the first maxillary molars and then exited out of the top of the head 
where the skin was removed from the top of the skull. The needle was removed from inside the cannula and then the cannula was placed near the affixed screw and covered with dental cement. The mouse was then given an i.p. dose of antibiotic and returned to its home cage to recover for 2 days. When the mouse had recovered from the anesthesia, the cannula was flushed to make sure it was not blocked and any blood inside was washed out.

\section{Taste Reactivity}

Mice were tested in a custom-made taste reactivity chamber. The chamber was a specially made, transparent, round Plexiglas chamber with a diameter of 4.5 inches. It sat on a base that elevated it 9.25 inches above the counter and contained a mirror so that the animal could be seen from all angles. The chamber was cleaned after the conclusion of each testing session to remove both traces of the previous animal and the taste solution used.

After recovery from the intra-oral cannula placement surgery, each mouse was put into the Taste Reactivity Chamber for two twenty-minute training sessions (Days 3 and 4) on two consecutive days to allow the mice to acclimate to the new environment. During the twenty-minute session, tubing connected to a 25 -gauge needle on a $10 \mathrm{cc}$ syringe was connected to the cannula on the mouse and water was forced into the cannula via a syringe pump (Harvard apparatus) with a flow rate of $0.10 \mathrm{ml} / \mathrm{min}$. The pump was turned on for 15 seconds and DI water was administered, then turned off for the next 45 seconds and repeated continuously throughout the rest of the training session. On the fifth day, either a taste solution or water was then forced into the cannula and the experiment was repeated in the same manner as in training. During this test, the animal was videotaped and the orofacial responses were then observed at a later date. Two hours after the initiation of the experimental video trial, the mouse was perfused and the brain tissue was processed for immunolocalization of $c$-fos. A summary of the procedure can be found in Chapter 3.

\section{Taste Reactivity Behavior Scoring}

A blind observer (Z.G.) reviewed all videotapes that were recorded previously during taste reactivity testing. After each of the 20 trials, the following behaviors were recorded on a worksheet if they occurred in a trial: paws to mouth, paw shakes, rearing, chin rubs, spitting, jumps, mouth movements, body grooming, and face grooming. 


\section{Histology}

\section{C-fos Immunohistochemistry with Nickel Intensified-3,3'Diaminobenzidine (DAB)}

Mice were anesthetized with $25 \%$ urethane and perfused with $50 \mathrm{~mL}$ of $0.1 \mathrm{M}$ phosphate-buffered saline (PBS) followed by $50-100 \mathrm{~mL}$ of $10 \%$ formalin solution (Fischer Scientific, Pittsburgh, PA). Brains were removed and post-fixed from 1-3 days in neutral-buffered formalin and then cryoprotected in a $20 \%$ sucrose $/ 10 \%$ glycerol solution. The brains were then frozen and sectioned on a freezing microtome. Free floating 40-micron coronal sections were placed into $0.1 \mathrm{M}$ phosphate buffer (PB). Every section in the area of the PBN was collected and only every other section was processed for $c$-fos immunohistochemistry, which was then performed on it. The floating slices were washed in $0.1 \mathrm{M}$ phosphate buffer, quenched with $30 \%$ hydrogen peroxide $(\mathrm{H} 2 \mathrm{O} 2)$ for 30 minutes, washed again, and then placed overnight in a primary solution containing anti $c$-fos antibody (Santa Cruz, Santa Cruz, CA) at a 1:1000 dilution, along with Normal Goat Serum and $0.8 \%$ Triton X. The sections were then rinsed again in $0.1 \mathrm{M} \mathrm{PB}$ and placed into secondary donkey anti-rabbit antibody (Rockland, Gilbertsville, PA) solution at a 1:50 dilution. The slices were then washed and placed into a rabbit peroxidaseantiperoxidase (PAP) solution (Covance, Princeton, NJ) at a 1:500 dilution. Lastly, sections were incubated in a nickel-DAB solution for 10 minutes, $\mathrm{H} 2 \mathrm{O} 2$ was then added to the nickel-DAB solution for 10 minutes, rinsed in $\mathrm{PB}$, mounted onto slides, dried at the minimum of overnight, dehydrated, and cover-slipped. A summary of this process can be found in Chapter 4.

\section{Cresyl Violet Staining}

The remaining sections not used for $c$-fos immunohistochemistry staining were mounted onto slides, dried, stained with cresyl violet, and cover-slipped. 


\section{CHAPTER 3. BEHAVIORAL ANALYSIS OF TASTE REACTIVITY AND TWO-BOTTLE PREFERENCE TESTS TO TASTE STIMULI IN C57BL/6J MICE}

This chapter covers the behavioral experiments from Specific Aim 1- to use methods to investigate sensitivity to sweet and umami compounds, including synergistic mixtures to determine if sweet and umami stimuli are similarly preferred by and elicit similar behavioral patterns in $\mathrm{B} 6$ mice. The following analysis includes taste reactivity and preference data.

\section{Introduction}

\section{Taste}

There are three behavioral divisions or aspects of taste (Spector, 2000; Spector \& Glendinning, 2009; St. John, 2008). The first aspect is "stimulus identification" that helps organisms to tell the difference between several varieties of different taste compounds via physiological interaction with the taste buds on the tongue. It also contributes to the survival of the organism by either enabling it to inherently identify important compounds (i.e. salt) or by learning through prior experiences (i.e. "sugar gives me energy or this bitter compound made me feel sick") of consuming a taste compound (Spector, 2000). The second "ingestive motivation" aspect consists of the behaviors that make the organism either consume a substance or instead produces a rejection reflex that causes the animal to spit out the substance when it hits the tongue in order to actively avoid a taste compound (Spector, 2000). The second aspect can be further subdivided into two parts: "appetitive behavior" and "consummatory behavior" (Spector, 2000; St. John, 2008). "Appetitive behavior" consists of the battery of behaviors that an organism goes through to approach a taste stimulus (Spector, 2000). The other portion of ingestive motivation, "consummatory behavior is the very last of the battery of behaviors that is produced by contact with a stimulus" (Spector, 2000; St. John, 2008). Lastly, the "digestive preparation" aspect consists of the ability of the taste compound to produce physiological autonomic responses to the taste compound such as saliva production and gastrointestinal responses (Spector, 2000; St. John, 2008). I employed two behavioral tests, two-bottle and taste reactivity tests, to investigate two of these divisions of taste: stimulus identification and both aspects of ingestive motivation.

\section{Preference Tests}

Two-bottle preference tests examine both the stimulus identification and both portions of the ingestive motivational division of taste (Spector, 2000; St. John, 2008). They have been used for decades to examine consumption and preference for a variety of taste compounds in both rats and mice. In order to perform this test, the animals were 
individually housed in their home cages and 2 bottles (with sipper tubes) are placed in the area in which their normal water bottle used to be (Tordoff \& Bachmanov, 2002). One bottle was filled with water and the other bottle was filled with one of the experimental solutions being tested (Tordoff \& Bachmanov, 2002). Every 24-hours the amount of liquid in each tube is measured, recorded, and then the bottles were replaced, with their left-right position switched to prevent a side bias, which has previously been found in the strain of mice we used in this experiment, B6 (Bachmanov et al., 2002; Lush, 1984; Tordoff \& Bachmanov, 2002). This high-throughput behavioral test was one I utilized to determine a basic overview of what taste stimuli were found aversive, neutral, or preferred by B6 mice in our laboratory.

\section{Taste Reactivity}

Taste reactivity tests examine only the consummatory subdivision of the ingestive motivational portion of taste (Spector, 2000; St. John, 2008). Taste reactivity tests have been performed in rodents, primates, and human infants. This test consists of dropping a variety of taste compounds into the mouth (of human infants or primates) or using an intraoral cannula in the mouth (rodents) to administer taste compounds. Then immediately following taste stimulation with a taste compound, the series of facial expressions that are elicited from the organism are recorded, observed, and analyzed.

In the 1970s, Steiner published taste reactivity studies using human babies as subjects, before their first feeding, so they would be almost entirely naïve in regards to taste solutions, except for what had made its way into the amniotic fluid of its mother (Berridge, 2000; Steiner, 1974). He found that sweet solutions elicited hedonic behaviors such as the movement of the corners of the mouth "in a smile-like expression," which was often followed by licking motions and sucking sounds (Steiner, 1973, pg. 257). He also found that bitter compounds, such as quinine, elicited aversive behaviors such as a facial reaction "characterized by marked depression of the mouth angles, with simultaneous elevation of the central part of the upper lip," creating an "arch-like," mouth opening (Steiner, 1973, pg. 271). The elongated tongue was also easily seen and eventually produced spitting and an overall impression of disapproval of the taste stimulus (Steiner, 1973). Other behaviors produced included the gape, nose wrinkle, frown, grimace, head shake, and arm flail in response to bitter stimuli (Steiner, Glaser, Hawilo, \& Berridge, 2001). The rest of the primary taste qualities evoked behaviors in between those observed from sweet and bitter and it has been found that each of the five primary taste qualities does not elicit a unique combination of behavior patterns during taste reactivity (Berridge, 2000). Therefore, an observer cannot guess what substance is being consumed, but can most likely guess the palatability of it (Steiner et al., 2001). Steiner was also able to produce the same behaviors in infants who were born with hydrocephaly and anencephaly (Steiner, 1973), and also in premature infants who were born at seven months gestation (Steiner, 1979). This discovery shows that these facial expressions are produced by the brainstem and are not learned, but innate (Steiner, 1979). 
Human adults are also capable of showing the same facial expressions in response to different taste stimuli, that Steiner found in infants. However, this is much harder to study since adults have had years to learn how to control their facial expressions and do not always display their true responses to a taste stimulus (i.e. the pursing of lips at a sour stimulus) (Berridge, 2000). Steiner was only able to successfully investigate the gustofacial reflexes of a few specific groups of adolescents and adults in a similar manner as he used in the studies performed in infants. These groups include blind adolescents, adolescents having Usher's syndrome, cognitively disabled adolescents and adults, and a population of Alzheimer's patients (Perl, 1992; Steiner, 1979).

Other animals exhibit taste reactivity behaviors, similar to ones found in human infants. These behaviors have been identified and studied in primates (Steiner et al., 2001; Steiner, Glaser, 1984, 1995), rats (Grill \& Norgren, 1978a, 1978b), and hamsters (Brining, Belecky, \& Smith, 1991). There has also been some work in chickens showing taste behaviors being elicited from primary taste stimuli, but these behaviors are harder to compare to the behaviors produced by the other different types of animals investigated (Ganchrow, Steiner, \& Bartana, 1990). In primates, many different species have been studied including the chimpanzee, orangutan, gorilla, rhesus monkey, white tufted marmoset, Humboldt's night monkey, and the mongoose lemur (Steiner et al., 2001). Tongue protrusions in response to sucrose along with gapes and head shaking in response to quinine were the taste reactivity behaviors elicited by all of the species of monkeys tested (Steiner et al., 2001). The taste reactivity behaviors of "smiling" and "lip smacking" to sucrose, "lip pursing" and sometimes "eye scrinching" to citric acid, or "frowning" and "nose wrinkling" to quinine were produced by only humans, chimpanzees, gorillas, and orangutans (Steiner et al., 2001). The taste reactivity behavior of "grimacing" that is produced by quinine was only displayed by chimpanzees, gorillas, and orangutans (Steiner et al., 2001). It was also found that the specific taste reactivity behaviors exhibited by the monkeys studied depended on how they were evolutionarily related to each other (Steiner et al., 2001).

Although the behaviors elicited are not exactly the same as in primates and humans, rodents also exhibit taste reactivity behavior. Rats and hamsters have been the experimental animals of choice. Grill and Norgren found that in rats, sucrose elicited a very consistent pattern of mouth movements, tongue protrusions and lateral tongue movements (Grill \& Norgren, 1978a, 1978b) and paw licking (Berridge, Grill, \& Norgren, 1981; Grill \& Berridge, 1985), while quinine elicits an entirely different pattern consisting of gapes, chin rubs, headshakes, and forelimb flails, with occasional lateral tongue movements (Grill \& Norgren, 1978a). The responses to $\mathrm{NaCl}$ and hydrochloric acid $(\mathrm{HCl})$ were found to be similar to sucrose (Grill \& Norgren, 1978a). The responses to a battery of umami stimuli in rats produced a high number of ingestive responses and a comparatively low number of aversive responses, with MSG producing the highest number of ingestive responses (Grill \& Flynn, 1987; Grill, Flynn, \& Schwartz, 1987). Rat pups also demonstrate some taste reactivity behaviors between the ages of 1-4 days old to sweet, acidic, and bitter stimuli (Ganchrow, Steiner, \& Canetto, 1986). Hamsters produced the same taste reactivity behaviors in response to a series of three different sweet stimuli and three different bitter stimuli that were also observed by Grill and 
Norgren in rats (Brining et al., 1991). Hamsters' overall responses differed from rats relative to acids and salt stimuli (Brining et al., 1991). Acids produced mouth movements, tongue protrusions, and lateral tongue protrusions, which are similar at first to rats (ingestive), but later on during the time course of the experiment, acids started producing gapes and smaller amounts of forelimb flailing, fluid rejection, and aversive posturing (Brining et al., 1991). The three different salt stimuli produced the same amount of ingestive behaviors as seen with the acid stimuli, but produced both a higher quantity of aversive behaviors and evoked the two other aversive behaviors of locomotion and chin rubbing (Brining et al., 1991). In response to three bitter stimuli, hamsters produced almost entirely aversive taste reactivity behaviors and only a small amount of ingestive behaviors (lateral tongue protrusions and tongue protrusions) (Brining et al., 1991).

Several experimental manipulations have been used to study and modify taste reactivity behaviors in rats. Lesions to the ventromedial edge of the ventral pallidum/substantia innominata produce an increase in aversive taste reactivity behaviors in response to sucrose (Cromwell \& Berridge, 1993). Wild-type and decerebrated rats did not display any difference in taste reactivity behaviors (Grill \& Norgren, 1978b), but removal of structures that lead to partial thalamic degeneration produced aversion behaviors (similar to those in response to quinine) to not only quinine, but also sucrose, $\mathrm{NaCl}$, and $\mathrm{HCl}$ (Grill \& Norgren, 1978b). In one study, morphine and naltrexone were administered to rats and they were found to alter taste reactivity behaviors (Parker, Maier, Rennie, \& Crebolder, 1992). In response to quinine, morphine decreased the number of aversive behaviors and naltrexone did not have an effect on aversive behaviors (Parker, Maier, Rennie, \& Crebolder, 1992). In response to sucrose, morphine did not have an effect on ingestive behaviors and naltrexone decreased the number of ingestive behaviors (Parker, Maier, Rennie, \& Crebolder, 1992). On the other hand, a study that administered morphine alone to rats and then administered a solution containing both sweet and bitter components leaving a longer interval between morphine administration and taste reactivity testing, found that only the ingestive hedonic behaviors were increased, while aversive behaviors stayed the same (Doyle, Berridge, \& Gosnell, 1993). Sodium depletion in rats increases their ingestive hedonic taste reactivity behaviors and eliminated the aversive taste reactivity behaviors in response to salt solutions (Berridge, Flynn, Schulkin, \& Grill, 1984). In rodents, hunger (by either deprivation for 24 or 48 hours) increases the number of ingestive taste reactivity behaviors to sucrose, water, or a sucrose-quinine mixture (Berridge, 1991). However, if a rodent has just eaten, has been administered cholecystokinin (Grill, 1995), or received a glucose gastric load (Cabanac \& Lafrance, 1990), the number of ingestive taste reactivity behaviors that were elicited from sucrose (Cabanac \& Lafrance, 1990), water, and a sucrose-quinine mixture (Berridge, 1991) were reduced. Reducing the body weight of rats by $10 \%$ did not show this decrease in hedonic responses to sucrose after administration of a gastric load (Cabanac \& Lafrance, 1991). Also, administration of an i.p. injection of lithium chloride (LiCl) after sucrose administration during a taste reactivity test over multiple times also altered the taste reactivity responses to sucrose, and produced an increase in aversive behaviors depending on the number of exposures (Breslin, Spector, \& Grill, 1992). Lastly, treatment with the drug, chlordiazepoxide (a benzodiazepine) increased the number of 
hedonic ingestive taste reactivity behaviors and only modified the amount of aversive taste reactivity behaviors a small amount in rats (Berridge \& Treit, 1986).

In mice, only two different strains, FVB/NJ and ICR outbred, have been utilized in taste reactivity experiments to only a handful of taste stimuli (Kiefer, Hill, \& Kaczmarek, 1998; Travers, Herman, Yoo, \& Travers, 2007). Neither of these studies included any umami compounds. In this study, I wanted to measure taste reactivity behaviors in response to both basic taste stimuli and two umami stimuli in B6 mice to determine whether they had a palatable or aversive nature. In order to do this, I had to make some modifications to Grill and Norgren's original taste reactivity procedure to make it easier to perform in mice (Grill \& Norgren, 1978a). The taste reactivity chamber was made smaller, so the mice had less area to move around in. This made it easier to videotape their behaviors. I also added a lid (with holes in it) so that the mice could not jump out of the chamber during training and testing sessions. I also modified the list of behaviors quantified because not all of the taste reactivity behaviors, originally found in their experiment performed with rats (Grill \& Norgren, 1978a), were possible to see in B6 mice because of their significantly smaller size and dark pigmentation.

\title{
Materials and Methods
}

\section{Experiment 1: Two-Bottle Test}

\begin{abstract}
Animals
A total of 10 naïve C57BL/6J mice ( 5 males and 5 females) were used for these experiments. They were either breed in the animal colony or purchased from the Jackson Laboratory (Bar Harbor, ME). Prior to testing, animals were group housed and separated according to sex in standard plastic mouse cages $(28 \times 17.5 \times 13 \mathrm{~cm})$ with ad libitum access to normal dry pellet chow and water. At the time of testing, animals were approximately 3-5 months old.

\section{Testing Apparatus}

Mice were tested in individual plastic mouse cages with wire mesh grids and kept in the animal facility during the entire test. Two Pyrex glass test tubes with metal sipper tubes (bottles) inserted inside rubber stoppers were put side by side into spaces between the metal grid on the right side where food is normally placed and the rodent chow was placed where the water bottle would normally be. The metal sipper tubes of both bottles were placed exactly the same distance into the cage.
\end{abstract}

\section{Solutions}

Solutions used for the 2-bottle experiment in Aim 1 included: deionized (DI) water, $0.03 \mathrm{M}$ sucrose, $0.1 \mathrm{M}$ sucrose, $0.01 \mathrm{M}$ sodium saccharin, $0.1 \mathrm{M}$ sodium saccharin, 
$0.03 \mathrm{M}$ MPG+10 $\mu \mathrm{M}$ amiloride (amil), 0.3 M MPG+10 $\mu \mathrm{M}$ amil, $0.03 \mathrm{M} \mathrm{MPG+0.1 \textrm {M }}$ IMP+10 $\mu \mathrm{M}$ amil, 0.3 M MPG+0.1 M IMP+10 $\mu \mathrm{M}$ amil, 0.03 M MSG+10 $\mu \mathrm{M}$ amil, 0.3 $\mathrm{M}$ MSG+10 $\mu \mathrm{M}$ amil, 0.03 M MSG+0.01 M IMP+10 $\mu \mathrm{M}$ amil, 0.3 M MSG+0.01 M IMP+10 $\mu \mathrm{M}$ amil, 0.0001 M IMP, 0.01 M IMP, 0.00003 M QHCl, and 0.0001 M QHCl (Table 3-1). Each of the solutions was prepared fresh on the first day that each stimulus was presented to the mice during each 48-hr test exposure. Each mouse was given each of the 18 stimuli and a different stimulus was given every 48-hours, with breaks over the weekends in which the two bottles were only filled with water. Amiloride was added to the umami solutions to prevent binding to epithelial sodium channels in the taste buds.

\section{Two-Bottle Test Procedure}

The amount of each liquid consumed was weighed every 24-hours (during the light cycle) and solutions were refilled as needed. The bottles were then put back in the cage as described previously, except that the bottle positions were switched to prevent a possible side bias (Bachmanov et al., 2002; Lush, 1984; Tordoff \& Bachmanov, 2002). Each mouse was weighed every 48-hours. Each mouse was given ad libitum access to each solution for 48-hours and then the bottles were removed, cleaned, and refilled with a new solution.

\section{Analysis}

Mean Preference Ratio. During the two-bottle test, the amount of both water and solution consumed was measured every 24-hours during each 48-hour exposure to each solution. These recorded values were used to calculate the mean preference ratio ([( solution consumed day $1 /$ total liquid consumed day 1$)+($ solution consumed day 2/total liquid consumed day 2)]/2) of each taste solution.

Total Solution Consumed. During the two-bottle test, the amount of each taste solution (i.e. sucrose) consumed was recorded every 24-hours during each 48-hour exposure to each solution. For each animal, these recorded values were used to calculate the total solution consumed (solution consumed on Day $1+$ solution consumed on Day 2 ). Then the average was calculated for the total solution consumed from each animal tested with that solution.

Statistics. Friedman Repeated Measures Analysis of Variance with Dunn's posthoc tests were performed on both the calculated values for each taste stimulus tested: Mean Preference Ratios and Total Solution Consumed.

\section{Experiment 2: Taste Reactivity Test}

\section{Animals}

A total of 46 naïve C57BL/6J mice were behaviorally tested and video-recorded in a taste reactivity chamber with intra-oral stimulation, and also used for subsequent 
Table 3-1. All Taste Stimuli Tests in One Group of 10 Animls during the TwoBottle Test.

\begin{tabular}{|c|c|}
\hline \multicolumn{2}{|c|}{ Taste Stimuli Administered } \\
\hline $1^{\text {st }}$ Concentration & $2^{\text {nd }}$ Concentration \\
\hline DI Water & DI Water \\
\hline 0.03 M Sucrose & $0.1 \mathrm{M}$ Sucrose \\
\hline $0.01 \mathrm{M}$ Saccharin & $0.1 \mathrm{M}$ Saccharin \\
\hline $0.00003 \mathrm{M} \mathrm{QHCl}$ & $0.0001 \mathrm{M} \mathrm{QHCl}$ \\
\hline $0.03 \mathrm{M}$ MPG+amil & $0.3 \mathrm{M}$ MPG+amil \\
\hline $0.03 \mathrm{M}$ MPG+0.01 M IMP+amil & $0.3 \mathrm{M}$ MPG+0.01 M IMP+amil \\
\hline $0.03 \mathrm{M}$ MSG+amil & $0.3 \mathrm{M}$ MSG+amil \\
\hline $0.03 \mathrm{M}$ MSG+0.01 M IMP+amil & $0.3 \mathrm{M}$ MSG+0.01 M IMP+amil \\
\hline $0.0001 \mathrm{M}$ IMP & $0.01 \mathrm{M}$ IMP \\
\hline
\end{tabular}


$c$-fos studies. Of this total (46), 35 mice were used for histological analysis in the $c$-fos studies $(\mathrm{H} 2 \mathrm{O}$ n=4, MPG+amil n=5, MPG+IMP+amil n=6, QHCl=7, SAC n=7, SUC n=7) and taste reactivity behavior was analyzed from video files in 25 mice $(\mathrm{H} 20 \mathrm{n}=2$, MPG+amil n =4, MPG+IMP+amil n=6, QHCl n=9, SAC n=2, SUC n=2). The reasons that 11/46 animals were not used for analysis of $c$-fos were due to errors with the histology; these are detailed later (Chapter 4, Materials and Methods). The reasons for using even smaller groups for analysis of taste reactivity (except $\mathrm{QHCl}$; see below) were chiefly technical in nature: a change to a smaller chamber design and technical difficulties with the camera. It is important to emphasize that although chamber conditions were varied somewhat within groups, the $c$-fos experiments should not be affected since stimulus delivery was through the intra-oral cannula, which was the same in all iterations of the experimental chamber design. In two mice stimulated with $\mathrm{QHCl}$, the histology was not usable, although I included the behavior in the taste reactivity results.

Both males and females $(\mathrm{H} 20=2$ males, $\mathrm{QHCl}=6$ males and 3 females, $\mathrm{SUC}=2$ females, $\mathrm{SAC}=1$ male and 1 female, $\mathrm{MPG}+$ amil $=4$ females, $\mathrm{MPG}+\mathrm{IMP}+$ amil $=6$ females) were used for these experiments and were either bred in the animal colony or purchased from the Jackson Laboratory (Bar Harbor, ME). Prior to testing, animals were group housed and separated according to sex in standard plastic mouse cages $(28 \times 17.5 \times 13 \mathrm{~cm})$ with ad libitum access to normal dry pellet chow and water. At the time of testing, animals were approximately 3-5 months old.

\section{Testing Apparatus}

Mice were tested in a custom-made taste reactivity chamber, which was a specially made, transparent, round Plexiglas chamber with a diameter of 4.5 inches. It sat on a base that elevated it 9.25 inches above the counter and contained a mirror so that the animal could be seen from all angles. The chamber was cleaned after the conclusion of each testing session to remove both traces of the previous animal and the taste solution used.

\section{Solutions}

Solutions used were $0.01 \mathrm{M}$ sodium saccharin, $1 \mathrm{M}$ sucrose, $0.003 \mathrm{M}$ QHCl, 0.1 M MPG $+10 \mu \mathrm{M}$ amil, $0.1 \mathrm{M}$ MPG+0.01 M IMP+10 $\mu \mathrm{M}$ amil, and DI water (Table 3-2). The solutions were prepared fresh and diluted with DI water, either the day before or the day of testing. Amiloride was added to the umami solutions to prevent binding to epithelial sodium channels in the taste buds.

\section{Taste Reactivity Training and Test}

After recovery from surgery, each mouse was put into the Taste Reactivity Chamber for two, twenty-minute training sessions on two consecutive days (Days 3 and 4) to allow the mice to acclimate to the new environment. During the twenty-minute session, tubing connected to a 25 -gauge needle on a $10 \mathrm{cc}$ syringe was connected to the 
Table 3-2. Taste Stimuli Tested, Number of Groups Tested, and Number of Animals in Each Group Used in the Taste Reactivity Test.

\begin{tabular}{lcc}
\hline Stimuli Tested & Group & $\mathbf{N}$ \\
\hline DI Water & 1 & 2 \\
$0.003 \mathrm{M} \mathrm{QHCl}$ & 2 & 7 \\
0.1 M Saccharin & 3 & 2 \\
1 M Sucrose & 4 & 2 \\
$0.1 \mathrm{M} \mathrm{MPG+amil}$ & 5 & 4 \\
$0.1 \mathrm{M} \mathrm{MPG+0.01} \mathrm{M} \mathrm{IMP+amil}$ & 6 & 6 \\
\hline
\end{tabular}


cannula on the mouse and water was forced into the cannula via a syringe pump (Harvard apparatus) with a flow rate of $0.10 \mathrm{ml} / \mathrm{min}$. The pump was turned on for 15 seconds and DI water was administered, then turned off for the next 45 seconds and repeated continuously throughout the rest of the training session (20 total exposures). On the fifth day, either a taste solution or water was then forced into the cannula and the experiment was repeated in the same manner as in training. During this test, the animal was videotaped and the orofacial responses were then observed at a later date. Two hours after the initiation of the trial, the mouse was perfused and the brain tissue was processed for $c$-fos. A summary of the procedure can be found in Table 3-3.

\section{Analysis}

The following behaviors were recorded the first time they occurred during the first 15 seconds (during which the mouse was actually being administered the taste stimuli via its intra-oral cannula) of each trial and the number of trials a behavior occurred in were summed and divided by the total number of trials (20).

Mouth Movements. This behavior was recorded each time the mouse was observed moving its mouth (without its paws in front of it) in response to taste stimuli.

Paws to Mouth. This behavior was recorded each time the mouse was observed using both hands to form a cup underneath its mouth.

Paw Shaking. This behavior was recorded each time the mouse was observed frantically shaking its front paws.

Facial Grooming. This behavior was recorded each time the mouse was observed brushing the sides of its face.

Body Grooming. This behavior was recorded when the animal was observed brushing or licking the area from its neck to the tip of its tail.

Rearing. This behavior was recorded each time the mouse was observed with its hind paws on the bottom of the chamber and its front paws on the side of the chamber.

Chin Rubbing. This behavior was recorded each time the mouse was observed rubbing its chin against the bottom of the chamber.

Spitting. This behavior was recorded when a stream of liquid was observed coming out of the mouse's mouth.

Jumping. This behavior was recorded when the animal was observed in the air with none of its paws touching the bottom of the chamber.

Statistics. Either one-way analysis of variance (ANOVA) tests followed by posthoc Bonferroni tests or Kruskal-Wallis tests followed by Dunn's post hoc tests were 
Table 3-3. Taste Reactivity Protocol.

\begin{tabular}{ll}
\hline Day & \multicolumn{1}{c}{ Procedure } \\
\hline 1 & Intra-Oral Cannula Surgery \\
2 & Recovery \\
3 & DI Water Taste Reactivity Training \\
4 & DI Water Taste Reactivity Training \\
5 & Taste Reactivity Test \\
\hline
\end{tabular}


performed for each of the nine behaviors elicited by each of the taste stimuli. When using the ANOVA statistical test, the following two factors are assumed: that the data are normally distributed and the variances for each group are equal. In order to determine if these were indeed true, they were tested. If either one of these tests failed, the nonparametric equivalent, Kruskal-Wallis one-way analysis on ranks was used instead that does not make the same assumptions.

\section{Results}

\section{Experiment 1: Two-Bottle Tests}

Mice were tested with a battery of taste stimuli including DI water, two concentrations of sucrose, two concentrations of saccharin, two concentrations of MPG+IMP+amil, two concentrations of MSG+IMP+amil, two concentrations of MPG+amil, two concentrations of MSG+amil, two concentrations of IMP, and two concentrations of $\mathrm{QHCl}$. In general, mice preferred the sweet stimuli to all other tastants, while umami stimuli were preferred compared to DI water. As expected, mice drank equally and indiscriminately between the two DI water tubes (sign of indifference) and avoided the bitter stimuli relative to water.

Preference (Chi-square $=126.604, \mathrm{df}=16$ degrees of freedom, $\mathrm{p}=<0.001)$ and consumption (Chi-square $=123.643, \mathrm{df}=16, \mathrm{p}=<0.001$ ) of each taste stimulus was compared to water and compared each stimulus to each other using a Friedman Repeated Measures Analysis of Variance with Dunn's post-hoc tests. Mice had a significantly higher preference for $0.1 \mathrm{M}$ sucrose, $0.03 \mathrm{M}$ sucrose, $0.01 \mathrm{M}$ saccharin, $0.03 \mathrm{M}$ MSG+IMP, 0.3 M MSG+IMP, and 0.01 M IMP compared to the water average (obtained from the two 48-hour water intervals) (Figure 3-1A). Mice significantly preferred $0.1 \mathrm{M}$ sucrose to $0.01 \mathrm{M}$ saccharin, 0.3 M MPG, 0.3 M MPG+IMP, 0.03 M MSG, and 0.0001 M IMP (Figure 3-2A). Mice also significantly preferred $0.01 \mathrm{M}$ saccharin to $0.0001 \mathrm{M}$ IMP and 0.3 M MPG+IMP (Figure 3-3). Mice also significantly preferred $0.03 \mathrm{M}$ sucrose, $0.1 \mathrm{M}$ sucrose, $0.01 \mathrm{M}$ saccharin, 0.3 M MSG, 0.03 M MSG+IMP, 0.3 M MSG+IMP and 0.01 M IMP to $0.00003 \mathrm{M} \mathrm{QHCl}$ (Figure 3-4A). Mice significantly preferred $0.03 \mathrm{M}$ sucrose, $0.1 \mathrm{M}$ sucrose, $0.01 \mathrm{M}$ saccharin, $0.03 \mathrm{M}$ MPG, $0.03 \mathrm{M}$ MPG+IMP, 0.3 M MSG, 0.03 M MSG+IMP, and 0.3 M MSG+IMP, and 0.01 M IMP to $0.0001 \mathrm{M} \mathrm{QHCl}$ (Figure 3-4B).

Mice had a significantly higher consumption (in $\mathrm{mL}$ ) of $0.1 \mathrm{M}$ Sucrose, $0.3 \mathrm{M}$ MPG, 0.3 M MSG, and 0.3 M MSG+IMP compared to the water average (Figure 3-1B). Mice significantly consumed more $0.1 \mathrm{M}$ sucrose than $0.03 \mathrm{M}$ MPG, $0.03 \mathrm{M}$ MSG, and 0.0001 M IMP (Figure 3-2B). Mice significantly consumed more 0.3 M MSG+IMP compared to $0.03 \mathrm{M}$ MPG, 0.03 M MPG+IMP, 0.03 M MSG, 0.03 M MSG+IMP, and 0.0001 M IMP (Figure 3-5A). Mice significantly consumed more $0.3 \mathrm{M}$ MSG compared to $0.0001 \mathrm{M}$ IMP and $0.03 \mathrm{M}$ MSG (Figure 3-5B). The mice showed significantly lower consumption of $0.00003 \mathrm{M} \mathrm{QHCl}$ compared to $0.03 \mathrm{M}$ sucrose, $0.1 \mathrm{M}$ sucrose, $0.01 \mathrm{M}$ 

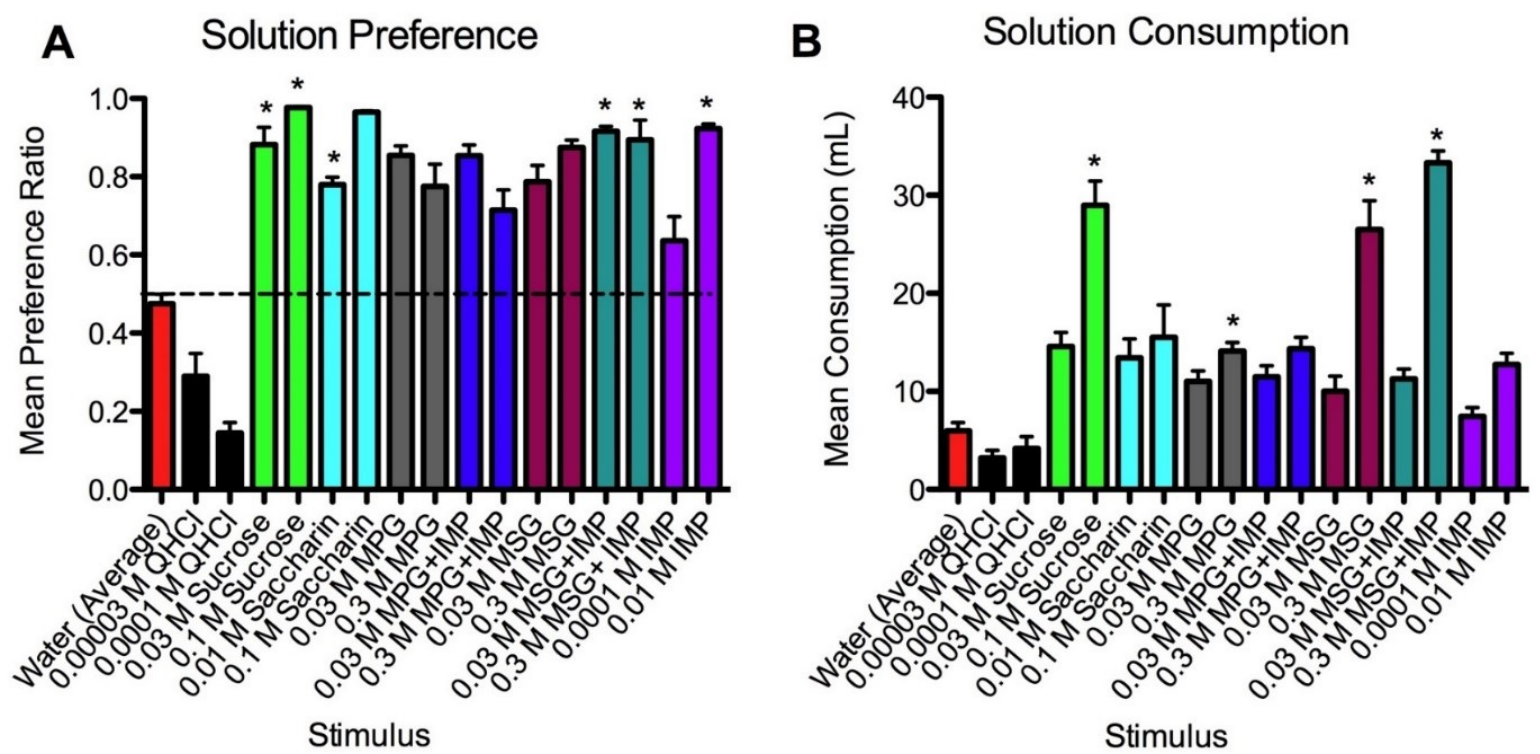

Figure 3-1. Overall Comparison of Taste Solution Preference and Consumption of Sweet, Umami, and Bitter Stimuli to Water to Determine Their Palatability in B6 Mice.

Notes: (A) Mean (+ SEM) preference ratio for B6 mice during exposure to a battery of taste stimuli. The mice show a significantly higher preference for both concentrations of sucrose, $0.01 \mathrm{M}$ saccharin, both concentrations of MSG+IMP, and 0.01 M IMP compared to water. (B) Mean (+ SEM) solution consumption for B6 mice during exposure to a battery of taste stimuli. B6 mice had a significantly higher consumption (in $\mathrm{mL}$ ) of $0.1 \mathrm{M}$ sucrose, $0.3 \mathrm{M}$ MSG, 0.3 M MSG+IMP, and 0.3 M MPG compared to water. Dotted line indicates indifference to a taste stimulus. ${ }^{*} \mathrm{P}<0.05$. 

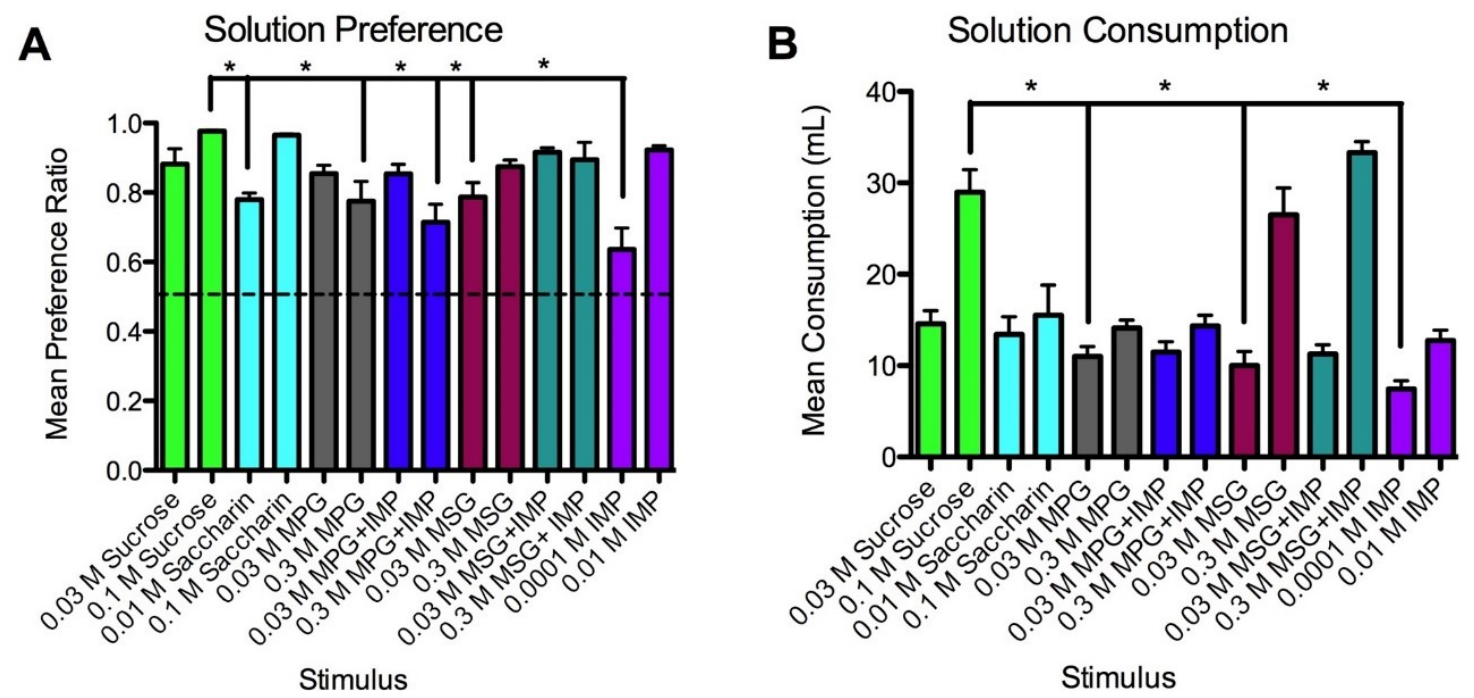

Figure 3-2. Comparison to Investigate the Similarity in Preference and Consumption Between All of the Sweet and Umami Stimuli in B6 Mice.

Notes: (A) Mean (+ SEM) preference ratio for B6 mice in response to sweet and umami stimuli. The mice show a significantly higher preference for $0.1 \mathrm{M}$ sucrose than $0.01 \mathrm{M}$ saccharin, 0.3 M MPG, 0.3 M MPG+IMP, 0.03 M MSG and 0.0001 M IMP. (B) Mean (+ SEM) solution consumption of B6 mice of sweet and umami stimuli. The mice had a significantly higher consumption of $0.1 \mathrm{M}$ sucrose compared to $0.03 \mathrm{M} \mathrm{MPG}, 0.03 \mathrm{M}$ MSG, and 0.0001 M IMP. Dotted line indicates indifference to a taste stimulus. ${ }^{*} \mathrm{P}<0.05$. 


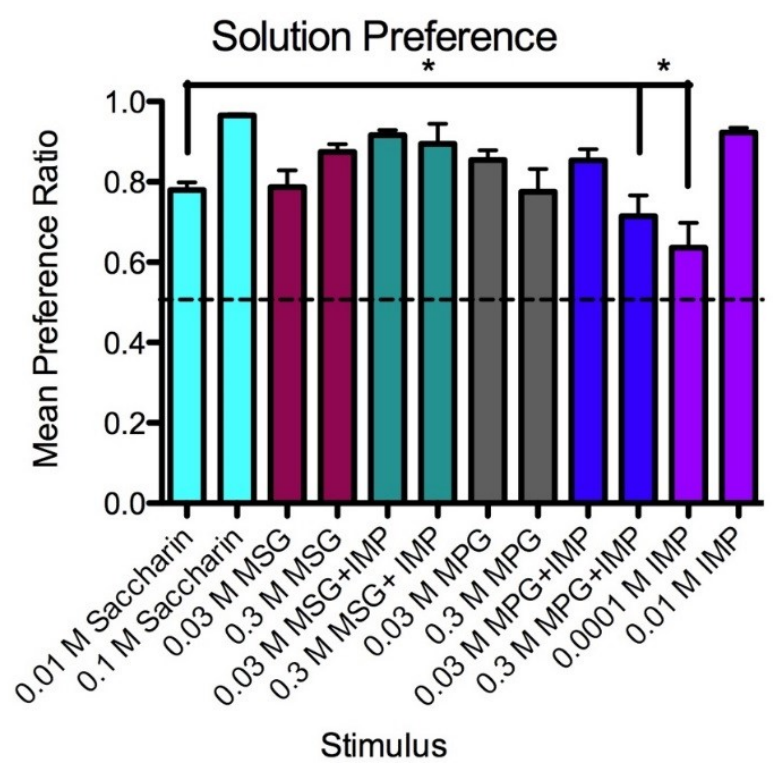

Figure 3-3. Comparison to Investigate the Similarity in Preference Between Saccharin and All of Umami Stimuli in B6 Mice.

Notes: Mean (+ SEM) preference ratio for B6 mice during exposure to saccharin and umami taste stimuli. B6 mice had a significantly higher preference of $0.01 \mathrm{M}$ saccharin compared to $0.03 \mathrm{M} \mathrm{MPG}+\mathrm{IMP}$ and $0.0001 \mathrm{M}$ IMP. Dotted line indicates indifference to a taste stimulus. $* \mathrm{P}<0.05$. 

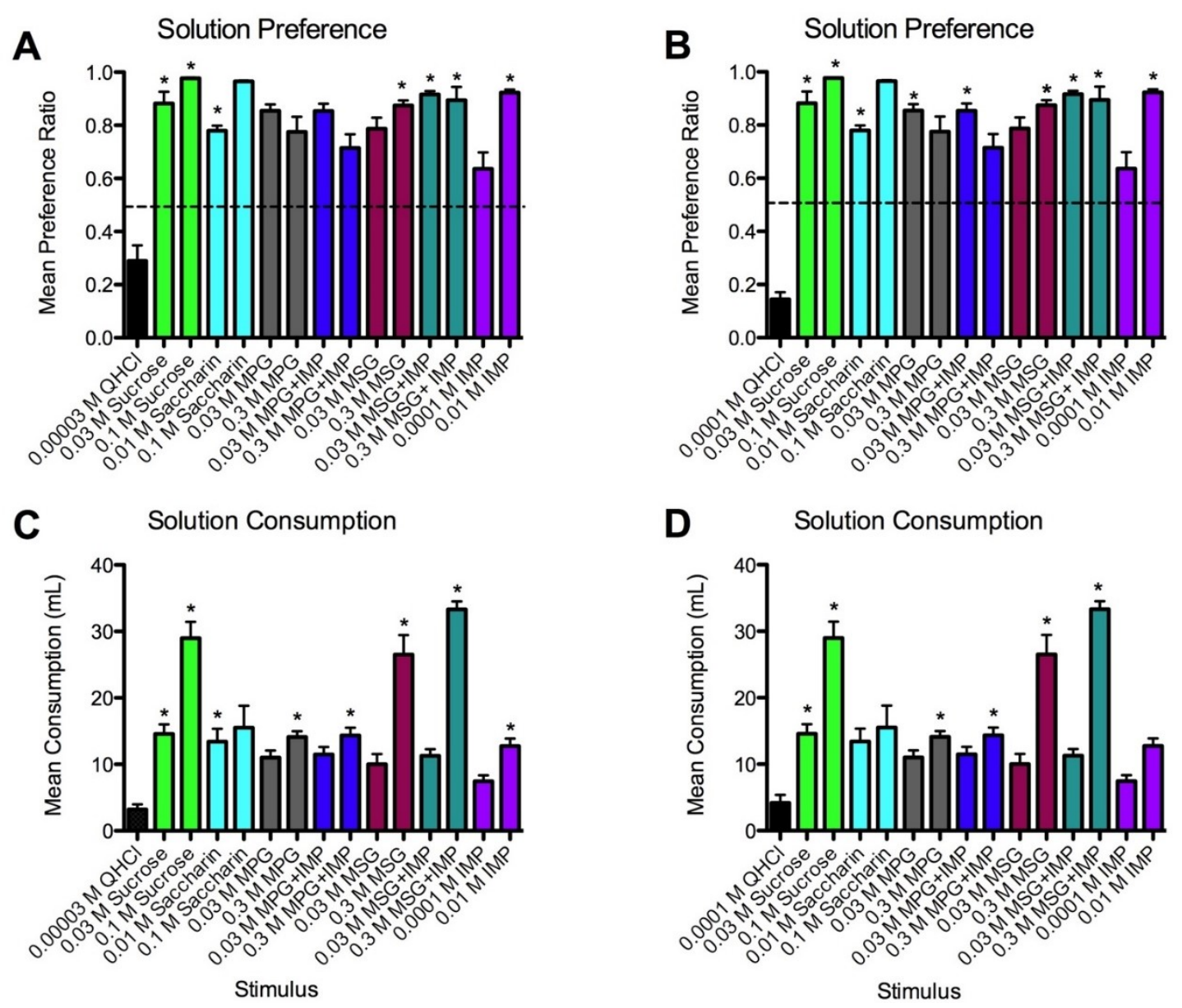

Figure 3-4. Comparison of Preference and Consumption of the Known Bitter Control QHCI to All Sweet and Umami Stimuli in B6 Mice.

Notes: (A) Mean (+ SEM) preference ratio for B6 mice during exposure to $0.00003 \mathrm{M}$ $\mathrm{QHCl}$ (bitter), sweet, and umami taste stimuli. The mice show a significantly lower preference for $0.00003 \mathrm{M} \mathrm{QHCl}$ compared to both concentrations of sucrose, $0.3 \mathrm{M}$ MSG, 0.03 M MSG+IMP, 0.3 M MSG+IMP, and 0.01 M IMP. (B) B6 mice had a significantly lower preference of $0.0001 \mathrm{M} \mathrm{QHCl}$ compared to both concentrations of sucrose, 0.01 M saccharin, 0.03 M MPG, 0.03 M MPG+IMP, 0.3 M MSG, 0.03 M MSG+IMP, 0.3 M MSG+IMP, and 0.01 M IMP. (C) Mean (+ SEM) solution consumption for B6 mice during exposure to $0.0001 \mathrm{M} \mathrm{QHCl}$ (bitter), sweet, and umami stimuli. The mice show significantly lower consumption of $0.00003 \mathrm{M} \mathrm{QHCl}$ compared to $0.03 \mathrm{M}$ sucrose, $0.1 \mathrm{M}$ sucrose, $0.01 \mathrm{M}$ saccharin, $0.3 \mathrm{M} \mathrm{MPG}, 0.3 \mathrm{M}$ MPG+IMP, 0.3 $\mathrm{M}$ MSG, 0.3 M MSG+IMP, and 0.01 M IMP. (D) B6 mice had a significantly lower consumption of $0.0001 \mathrm{M}$ QHCl compared to $0.03 \mathrm{M}$ sucrose, $0.1 \mathrm{M}$ sucrose, $0.3 \mathrm{M}$ MPG, 0.3 M MPG+IMP, 0.3 M MSG, and 0.3 M MSG+IMP. Dotted line indicates indifference to a taste stimulus. $* \mathrm{P}<0.05$. 

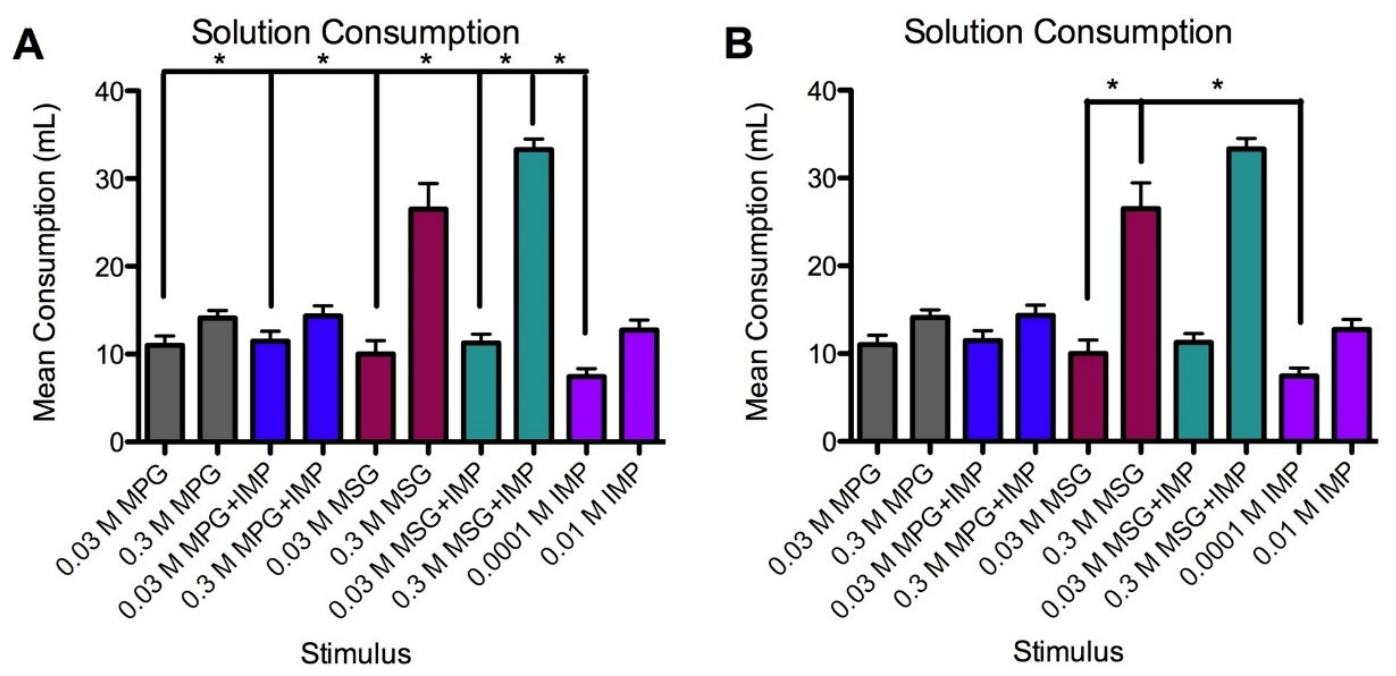

Figure 3-5. Comparison to Investigate the Similarity in Consumption Between All of the Umami Stimuli in B6 Mice.

Notes: (A) Mean (+ SEM) solution consumption for B6 mice during exposure to umami stimuli. The mice show significantly higher consumption of $0.3 \mathrm{M} \mathrm{MSG}+\mathrm{IMP}$ compared to $0.03 \mathrm{M}$ MSG, $0.03 \mathrm{M}$ MSG+IMP, 0.03 M MPG, 0.03 M MPG+IMP, and 0.0001 M IMP. (B) Mean (+ SEM) solution consumption for B6 mice during exposure to umami stimuli. The mice also significantly consumed more of $0.3 \mathrm{M} \mathrm{MSG}$ compared to 0.0001 M IMP and 0.03 M MSG. Dotted line indicates indifference to a taste stimulus. ${ }^{*} \mathrm{P}<0.05$. 
saccharin, 0.3 M MPG, 0.3 M MPG+IMP, 0.3 M MSG, 0.3 M MSG+IMP, and 0.01 M

IMP. (Figure 3-4C). Lastly, B6 mice had a significantly lower consumption of $0.0001 \mathrm{M}$ QHCl compared to $0.03 \mathrm{M}$ sucrose, $0.1 \mathrm{M}$ sucrose, 0.3 M MPG, 0.3 M MPG+IMP, 0.3 M MSG, and 0.3 M MSG+IMP. (Figure 3-4D).

\section{Experiment 2: Taste Reactivity Behavior}

\section{Overall}

Each mouse was put into a taste reactivity chamber and stimulated with only one of the following stimuli over the course of 20 minutes: DI Water, Sucrose, Saccharin, $\mathrm{MPG}+$ amil, $\mathrm{MPG}+\mathrm{IMP}+$ amil, and $\mathrm{QHCl}$. Nine taste reactivity behaviors were able to be identified. The number of occurrences [one time for each behavior during each of the 20 (15 second) stimulations during each test session] was quantified from the recorded sessions and analyzed.

\section{Consistency of TR Behavior Patterns during Stimulation with Each Taste Compound}

In general, if a behavior was elicited by a taste stimulus, it was quantified during the entire 20-minute trial, with a few exceptions. The first exception was paw shaking. All of the stimuli produced paw shakes consistently, except for in response to sucrose and saccharin (Figure 3-6A). Rearing was also elicited consistently throughout the test for all stimuli except for sucrose and saccharin, in which it did not occur until the end of the test on those tastants (Figure 3-6B). Chin rubbing only consistently occurred during the entire 20-minute trial in response to $\mathrm{QHCl}$ (Figure 3-6C). When spitting occurred, it was only produced consistently throughout the 20 -minute trial by one stimulus, $\mathrm{QHCl}$ (Figure 3-6D). When jumping occurred, it was towards the end of the test (Figure 3-6E) Lastly, facial grooming occurred consistently throughout the entire trial in response to all stimuli except for $\mathrm{QHCl}$, where it occurred at the beginning of the trial and then stopped completely (Figure 3-7D).

\section{Differences in TR Behavior Between Each Stimulus}

Every one of the nine behaviors was displayed in the course of testing all of the six stimuli, but none of the six taste stimuli elicited all nine of the behaviors during a testing session (Figure 3-8). This is illustrated in the overall summary graph that shows the percent of trials each behavior was observed in response to each of the six of the taste stimuli. Each taste stimulus elicited a variety of responses, with the exception of the two umami taste stimuli, MPG+IMP+amil and MPG+amil (Figure 3-8E and F), in which both elicited an overall similar variety of behaviors which included a similar percentage of occurrences all of the behaviors quantified except for chin rubbing (which did not occur at all in both stimuli). The majority of the responses to water (Figure 3-8A) were paws-to-mouth, paw shakes, rearing, face grooming, and mouth movements. In response to $\mathrm{QHCl}$ (Figure 3-8B), the same behaviors were exhibited as the other taste stimuli, but 
A

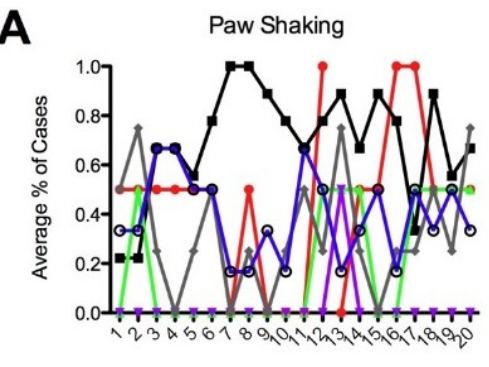

Trial

D

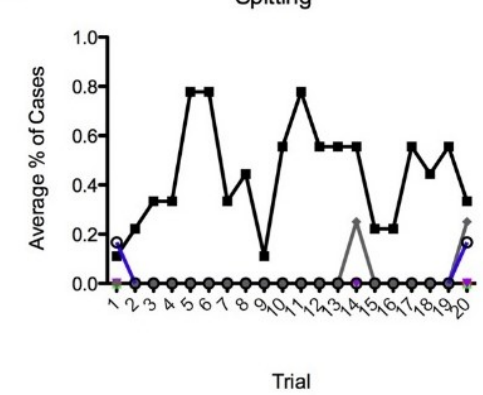

B

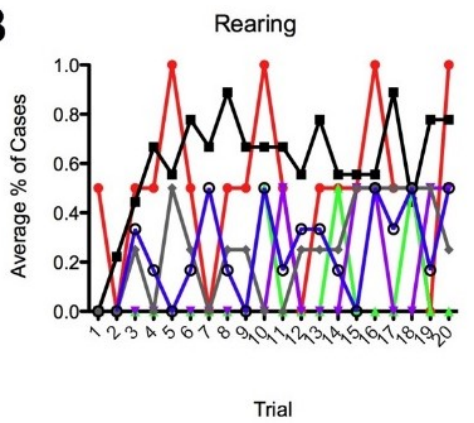

E

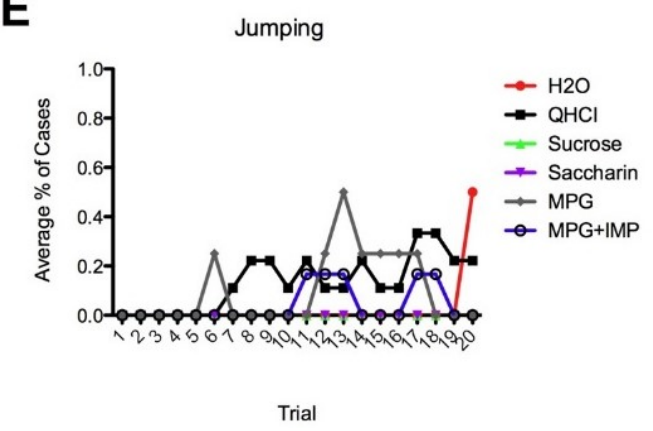

Figure 3-6. Non-Appetitive Taste Reactivity Behaviors in Response to All the Taste Stimuli and Their Occurrence over 20-Minute (1-Minute) Trials.

Notes: (A) All of the stimuli except for sucrose and saccharin elicited paw shakes during the majority of the 1-minute trials. (B) Rearing was produced by all the stimuli, but in sucrose and saccharin, it was not produced until the last half of the trials. (C) Chin rubbing only consistently occurred during the entire 20-minute trial in response to $\mathrm{QHCl}$. (D) Spitting only occurred consistently throughout all trials in response to $\mathrm{QHCl}$. (E) In the four taste stimuli ( $\mathrm{H} 2 \mathrm{O}, \mathrm{MPG}, \mathrm{MPG}+\mathrm{IMP}$, and $\mathrm{QHCl})$ that jumping is elicited, it occurred towards the end of the taste reactivity trial. 
A

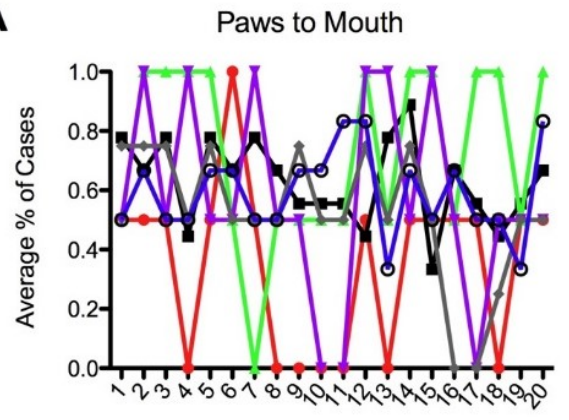

Trial

C

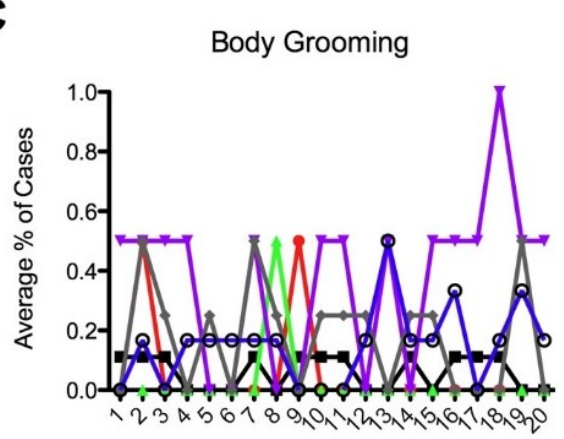

Trial
B Mouth Movements

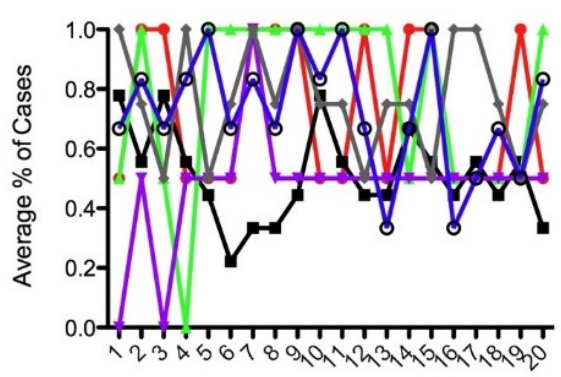

Trial

D

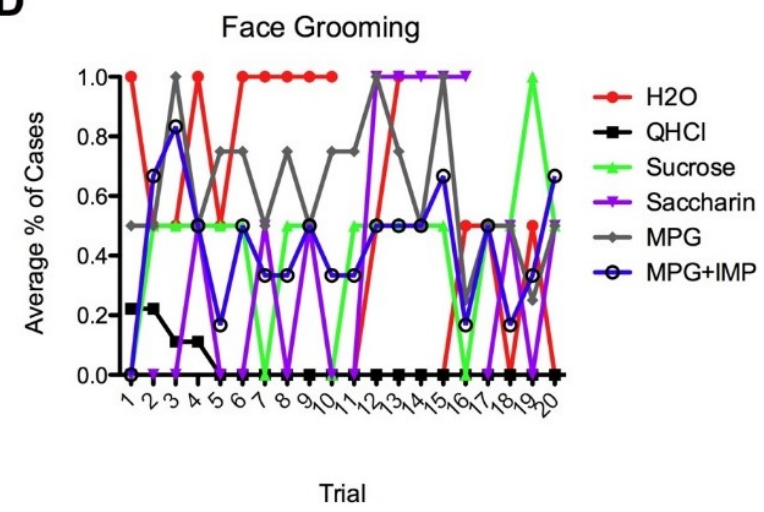

Figure 3-7. Appetitive Taste Reactivity Behaviors in Response to All the Taste Stimuli and Their Occurrence over 20-Minute (1-Minute) Trials.

Note: In A-C, all of the behaviors are observed throughout the 20-minute test, with the exception of facial grooming (D) during $\mathrm{QHCl}$ stimulation, which occurred at the beginning of the trials and then stopped completely. 

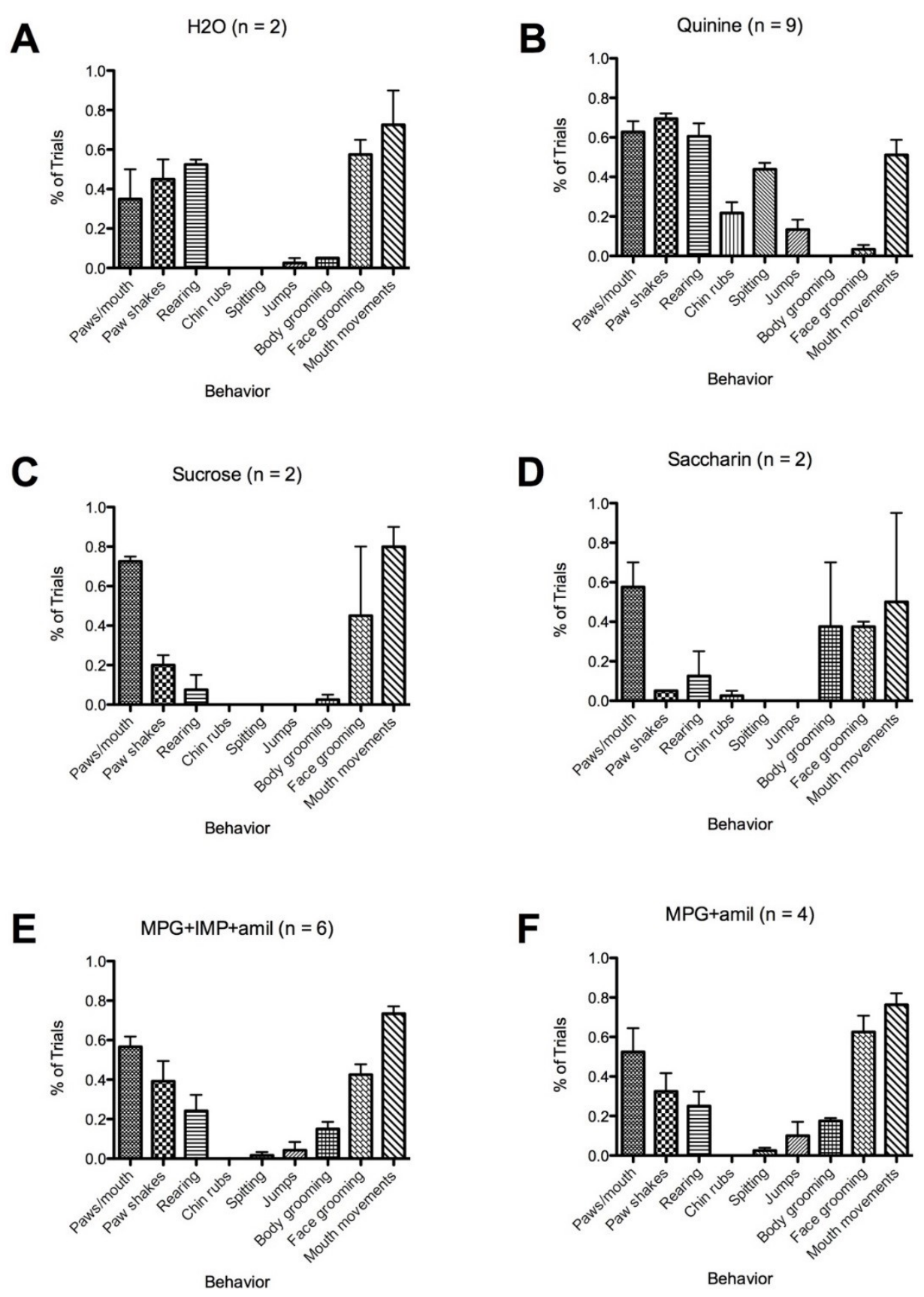

Figure 3-8. Overview of Taste Reactivity Behaviors in Response to Each of the Taste Stimuli.

Note: Each taste stimuli tested seems to evoke a different percent of each of the nine taste reactivity behaviors overall during a 20-minute testing session, except for the two umami stimuli, the synergistic mixture (E) MPG+IMP+amil and (F) MPG+amil. 
additionally, there was either an increase or the initial appearance of rearing, spitting, jumps, and chin rubs. In response to sucrose (Figure 3-8C), the majority of the behaviors exhibited were paws-to-mouth, mouth movements, and face grooming. In response to saccharin (Figure 3-8D), the majority of the behaviors mice exhibited were paws-tomouth, body grooming, face grooming, and mouth movements. In the three groups that only had two animals in each group: water, sucrose, and saccharin, individual graphs were made of the percentage of taste reactivity behaviors in response to those stimuli. The taste reactivity behaviors from each individual animal stimulated with water are shown in Figure 3-9A and B. The graphs from the individual animals stimulated with sucrose are shown in Figure 3-9C and D. Lastly, the graphs from the individual animals stimulated with saccharin are shown in Figure 3-9E and F. These figures illustrate that there is variability between the mice in how often they elicit the taste reactivity behaviors and accounts for the larger error bars in these smaller groups, but also demonstrates that the kinds of behaviors elicited by a stimulus are consistent, even if the levels are not entirely the same.

Either one-way analysis of variance (ANOVA) tests followed by post-hoc Bonferroni tests or Kruskal-Wallis tests followed by Dunn's post-hoc tests were performed for each of the nine behaviors elicited by each of the taste stimuli. All of the taste stimuli, except for $\mathrm{QHCl}$, produced body grooming (Figure 3-10A). The incidence of body grooming during stimulation with each of the six taste stimuli were significantly different, further analysis using the Dunn's post hoc test did not reveal which of the six taste stimuli were significantly different from each other $(\mathrm{df}=5, \mathrm{H}=13.819, \mathrm{p}=0.017)$ (Figure 3-10A). While all taste stimuli produced paw shaking in the mice, the percentage produced during saccharin $(\mathrm{df}(5,19), \mathrm{F}=8.446, \mathrm{p}<0.001)$, sucrose $(\mathrm{df}(5,19), \mathrm{F}=8.446$, $\mathrm{p}=0.014)$, MPG (df(5,19), F=8.446, $\mathrm{p}=0.018)$, and MPG+IMP $(\mathrm{df}(5,19), \mathrm{F}=8.446$, $\mathrm{p}=0.032$ ) stimulation was significantly lower than during $\mathrm{QHCl}$ stimulation (Figure 3-10B). There was a statistically significant difference between all of the tastant groups in eliciting chin rubs $(\mathrm{df}=5, \mathrm{H}=14.953, \mathrm{p}=0.011)$, but the Dunn's post hoc test analysis could not discern which individual tastants were different (Figure 3-10C). All of the taste stimuli produced facial grooming during stimulation, but the percentage that was displayed during stimulation with $\mathrm{QHCl}$ was significantly lower than the percentage displayed during stimulation with $\mathrm{MPG}(\mathrm{df}=5, \mathrm{H}=18.679, \mathrm{p}=0.002)$ (Figure 3-10D). The incidence of rearing in response to sucrose $(\mathrm{df}(5,19), \mathrm{F}=6.011, \mathrm{p}=0.020)$, saccharin $(\mathrm{df}(5,19), 6.011, \mathrm{p}=0.045)$, and MPG+IMP $(\mathrm{df}(5,19), \mathrm{F}=6.011, \mathrm{p}=0.017)$ was significantly lower than to $\mathrm{QHCl}$ (Figure 3-10E). Lastly, there was a significant difference found between the groups in the amount of spitting, but the Dunn's post-hoc test was not able to elucidate which groups were different from each other $(\mathrm{df}=5$, $\mathrm{H}=20.099, \mathrm{p}=0.001$ ) (Figure 3-10F). 
A

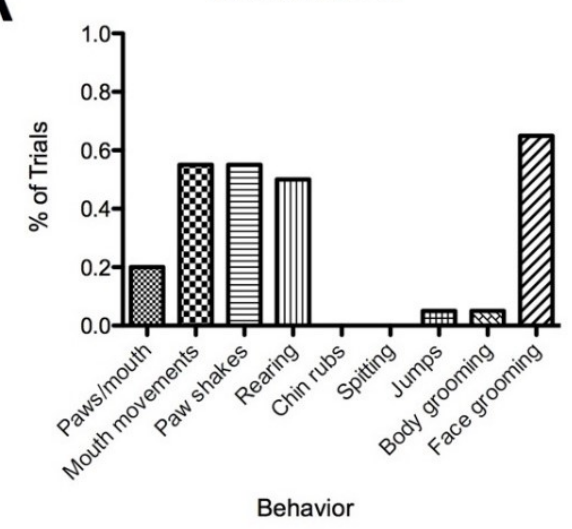

C

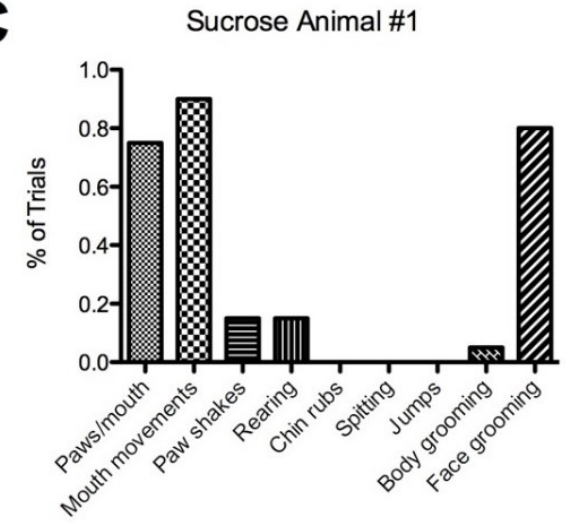

Behavior

E

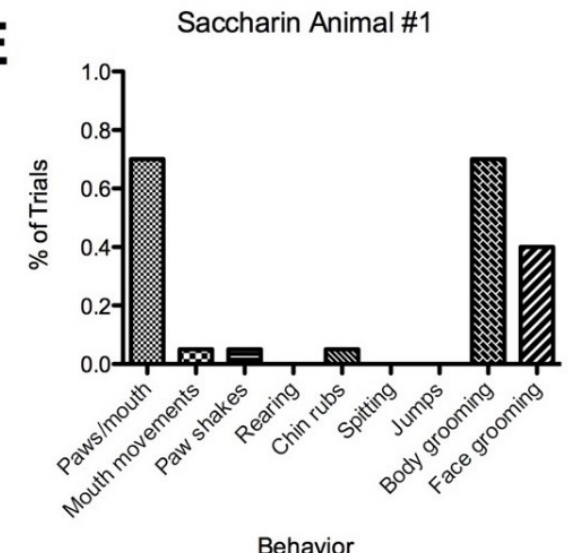

B

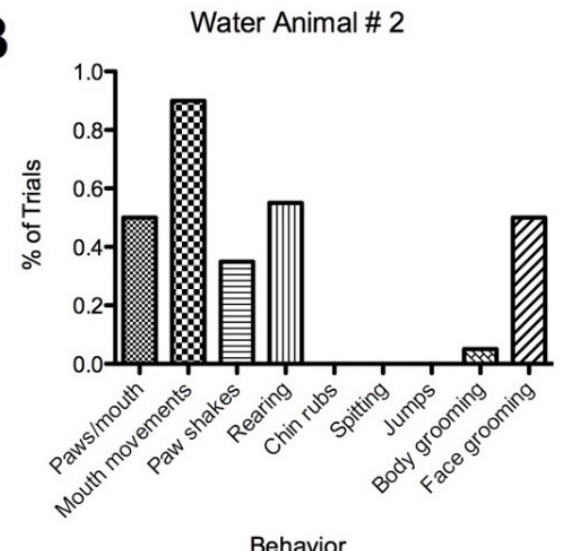

D

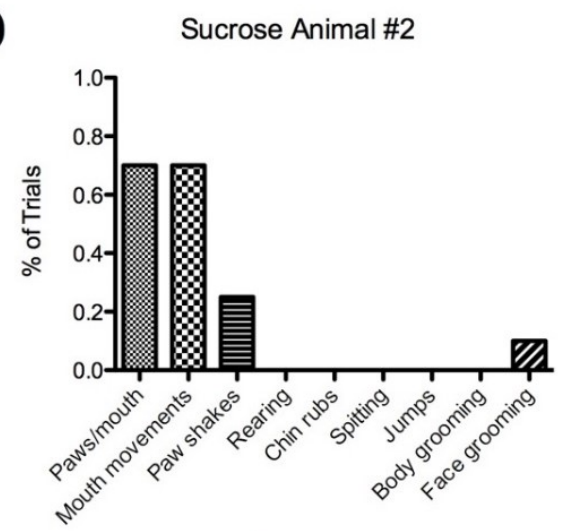

Behavior

$\mathbf{F}$

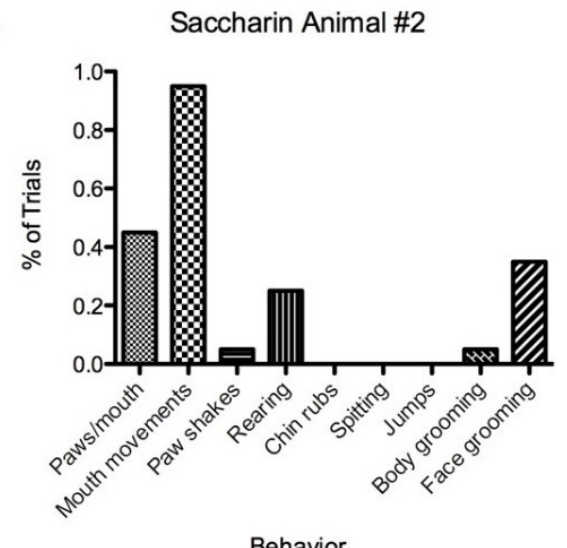

Figure 3-9. Taste Reactivity Behavior Responses of Individual Animals Stimulated with Water, Sucrose, and Saccharin.

Note: Illustrates the individual variability in the number of trials each behavior occurs in water-stimulated (A) and (B), sucrose-stimulated (C) and (D), and saccharin-stimulated (E) and (F) animals. 

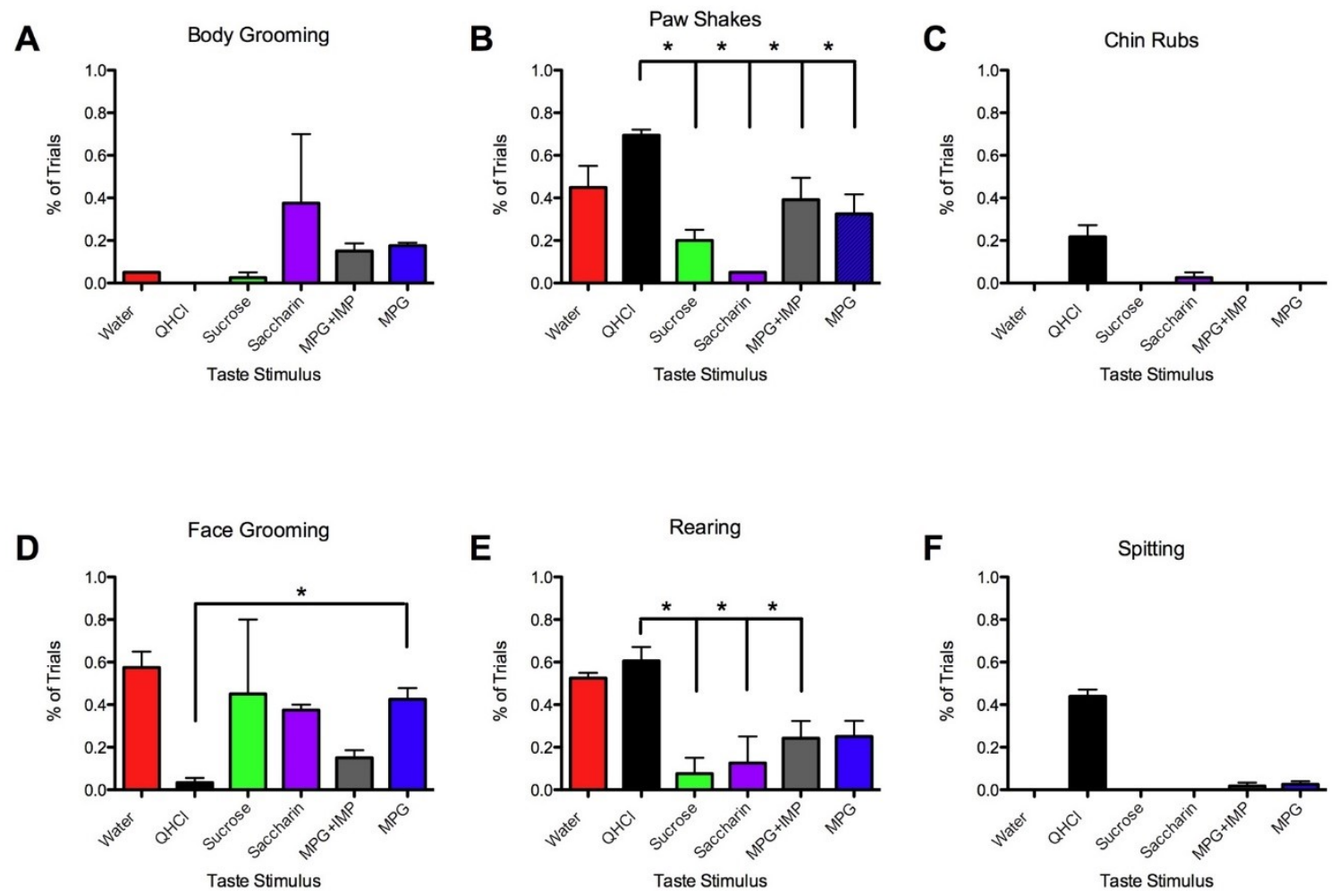

Figure 3-10. The Incidence of the Occurrence of 6 Different Taste Reactivity Behaviors during Stimulation with Each of the Different Taste Stimuli.

Notes: A) The incidence of body grooming during stimulation was significantly different between the six taste stimuli. B) The incidence of paw shaking during $\mathrm{QHCl}$ stimulation is significantly higher than with stimulation of sucrose, saccharin, the synergistic mixture (MPG+IMP) and MPG. There also seems to be a trend of the more bitter stimuli (QHCl and water) to have more paw shakes, followed by an intermediate amount occurring during stimulation with the two umami stimuli (MPG and MPG+IMP), followed by the smallest amount of paw shakes occurring during stimulation with the sweet stimulation (sucrose and saccharin). C) The incidence of chin rubbing was significantly different between the six taste stimuli. D) The incidence of facial grooming that occurs during stimulation with MPG is significantly higher than when the animal is stimulated with $\mathrm{QHCl}$. E) The incidence of rearing during stimulation with $\mathrm{QHCl}$ was significantly higher than during stimulation with sucrose, saccharin, and the synergistic mixture (MPG+IMP). F) The incidence of spitting during stimulation was significantly different between the taste stimuli. ${ }^{*} \mathrm{P}<0.05$. 


\section{Discussion}

\section{Experiment 1: Preference Tests}

In summary, we performed two-bottle tests with a battery of different taste stimuli on B6 mice to assess, which stimuli were preferred, and which were aversive. The B6 mice preferred all the sweet and umami compounds to water and avoided both concentrations of QHCl (Figure 3-1A). However, one of the umami stimuli, 0.3 M MSG+IMP, was consumed at the same level as sucrose (Figure 3-1B).

The large consumption (average total amount of solution consumed) and high preference ([(solution consumed day $1 /$ total liquid consumed day 1$)+($ solution consumed day $2 /$ total liquid consumed day 2$)] / 2$ ) of the two sweet stimuli (sucrose and saccharin) and some of the different types of umami stimuli (MSG, MPG, MPG+IMP, and IMP) found in this study indicate that they are highly palatable to this strain of mice. This pattern of preference has been found previously in this strain of mice with sucrose, saccharin (Bachmanov, Tordoff, et al., 2001), MSG, and IMP (Bachmanov et al., 2000). No other study has used MPG and combined MPG+IMP and MSG+IMP in two-bottle tests utilizing mice. There are no studies performed in rats using the same concentrations of umami stimuli that were used in this study. However, there are two studies in rats that used lower concentrations of umami stimuli and the rats seem to only prefer umami stimuli at lower concentrations compared to mice (Miura, Ooki, Kanemaru, \& Harada, 2014; Grill and Flynn, 1987). In the higher concentration of umami stimuli used in the rats, the rats showed a very low preference ratio, similar to the $0.0001 \mathrm{M} \mathrm{QHCl}$ used in this study (Grill and Flynn, 1987). This illustrates a possible species difference between mice and rats.

Based on both electrophysiological data (Tokita et al., 2012) and CTA data (Saites et al., 2015) obtained in the Boughter lab, I expected that the synergistic mixtures (MSG+IMP and MPG+IMP) used in this experiment would be preferred at a similar level as sucrose, since it seems like MPG+IMP is considered by mice both to taste similar and activate the same neurons. This was true for both concentrations of MSG+IMP, but not for MPG+IMP, as seen in Figure 3-2A and B. It is possible that the $\mathrm{K}+$ ion in the concentrations of MPG that were used made this amino acid bitter and therefore less preferred and consumed than both MSG and the sweet stimuli. I also expected that the synergistic mixtures would be preferred more than either MPG or MSG individually. This was true for both concentrations of MSG and MPG, as seen in Figure 3-2A and B. When each was combined with IMP, the preference ratio increased for both. Even though amino acids contain a similar caloric load as sugars, only one of these umami stimuli produced consumption similar to sucrose, the higher concentration of MSG+IMP, with the higher concentration of MSG not too far behind. Even though these umami stimuli were still highly preferred like the sugars were, the caloric nature did not seem to contribute to a high amount of consumption. 
The non-caloric sweetener, sodium saccharin only displayed similar preference to sucrose at the higher concentration tested $(0.1 \mathrm{M}$ saccharin) and not at the lower concentration tested ( $0.01 \mathrm{M}$ saccharin). This is most likely influenced by the non-caloric nature of saccharin via gastrointestinal feedback over the 48 hours that they had $a d-$ libitum access to this stimulus. Also, saccharin has been shown to possess a bitter aftertaste, which may have also contributed to the lower consumption of both concentrations of saccharin. Both concentrations of saccharin displayed similar preference and consumption to that of most of the umami stimuli. This reveals that there may be an aversive taste of these stimuli, possibly similar to the bitter aftertaste of saccharin, which may be preventing the mice from consuming them in a similar manner to sucrose. Also, the umami stimuli may produce some slightly negative post-ingestive feedback that could be resulting in a lower consumption. Perhaps, at lower concentrations than were tested in this study, more of the umami stimuli would be preferred and consumed in a similar manner as sucrose. The preference ratio of the higher concentration of quinine was similar to a previous study performed in B6 mice using the same $0.0001 \mathrm{M}$ concentration of quinine (Tordoff, 2007). Both concentrations of quinine were not preferred or consumed and were actively avoided compared to all of the other stimuli tested.

\section{Experiment 2: Taste Reactivity}

In summary, six taste stimuli were administered to mice via an intra-oral cannula during a taste reactivity test and nine behaviors were observed and quantified. All nine behaviors were consistently observed and were able to be recorded during the entire 20minute testing session, except in a few cases. Paw shaking (Figure 3-6A) and rearing (Figure 3-6B) were not displayed until near the end of the testing with sucrose and saccharin. QHCl was the only stimulus to consistently produce spitting (Figure 3-6D) in response to stimulation with it. QHCl also only produced facial grooming (Figure 3-7D) at the very beginning of the test, but this behavior quickly ceased after the first few trials. Only the two umami stimuli (MPG+amil and MPG+IMP+amil) exhibited a similar variety and amount of each of the different taste reactivity behaviors (Figure 3-8 E and F). $\mathrm{QHCl}$ produced the most disparate results among the stimuli and produced either: none (body grooming), the lowest level (face grooming), or the highest level of behaviors (paw shaking, chin rubbing, rearing, and spitting) (Figure 3-8 B).

The taste reactivity behaviors that could be quantified in this study had to be modified for both the small size of the mice and for the resolution of the video recording, which was not a high-speed unit. It was not possible to identify specific lateral tongue protrusions, tongue protrusions, paw pushing, and gaping as had been previously done in rats (Grill \& Norgren, 1978a), hamsters (Brining et al., 1991), and mice (Travers et al., 2007 ) in the recorded videos. These behaviors were therefore lumped together in one group as mouth movements.

The taste reactivity data revealed that in the B6 mouse, responses to aversive stimuli, such as quinine, are easily distinguished from other stimuli, by both the number 
and specific behaviors this bitter stimulus elicits. Quinine produced the most nonappetitive behaviors which included paw shaking (Figure 3-10B), chin rubbing (Figure 3-10C), rearing (Figure 3-10 E), and spitting (Figure 3-10F). It also produced a complete lack of body grooming (Figure 3-10A) and only a small amount of face grooming (Figure 3-10D). The behaviors that were observed were similar to the ones also produced by the FVB/NJ (Travers et al., 2007) and ICR outbred strains of mice (Kiefer et al., 1998) in response to quinine, except that chin rubbing was not exhibited at all by ICR outbred mice. It is difficult to compare our data to the ICR inbred mice study because of differing methodology in terms of quantifying the behaviors. In this study, it was recorded whether or not a particular behavior occurred only in a trial in response to a tastant in B6 mice, while Keifer et al. recorded every time a behavior occurred in the ICR outbred mice. However, the frequency of these behaviors varied between B6 and FVB/NJ mice. Paw shaking, paws to mouth, rearing, chin rubbing, spitting, jumping, and mouth movements were all produced more often in B6 mice than in the FVB/NJ strain. These behaviors either did not occur at all or in a smaller number in response to the other taste stimuli tested. Also, both a species and strain difference were discovered, which was either the presence or absence of the behavior, jumping. Jumping did not occur in hamsters, rats, and ICR outbred mice, but B6 mice and FVB/NJ mice displayed jumping during taste reactivity tests. We found that the B6 mice jumped enough when we started our study that we had to get a specially designed lid made with holes in it so that the mice could not jump out of the activity chamber during testing. We separated grooming into two categories, body and face grooming, and even though other investigators put all grooming activities in one category, the amount of grooming by the $\mathrm{B} 6$ mice was less when combined than in the FVB/NJ strain. This finding differs from what was found in rats in one study, where the same concentration of quinine produced face washing in 80 percent of the trials (Grill \& Norgren, 1978a). In another study, a lower level of facial grooming was found which was more similar to what we saw in the B6 mice (Kiefer et al., 1998). In hamsters, quinine elicits the same behaviors as we found in our study (except for jumping), just at higher levels, except for a decrease in paw shaking, most likely because they used a $0.1 \mathrm{M} \mathrm{QHCl}$ concentration compared to our $0.003 \mathrm{M} \mathrm{QHCl}$ concentration (Brining et al., 1991). These behaviors illustrate that all three species of rodents were trying to expel this stimulus from their mouth in several possible ways.

In rats, water stimulation produced paw shaking and facial grooming (Kiefer \& Dopp, 1989), which was similar to the behaviors produced in ICR outbred mice (Kiefer et al., 1998) and the B6 mice in this study. The response of ICR outbred mice to water produced both mouth movements and paw shaking, which was also seen in B6 mice during water stimulation. The main difference between the two strains is that the ICR mice produced a small amount of spitting in response to water, which was not seen in either the $\mathrm{B} 6$ and $\mathrm{FVB} / \mathrm{NJ}$ mice. The $\mathrm{B} 6$ and $\mathrm{FVB} / \mathrm{NJ}$ mice produced similar levels of paws to mouth, mouth movements, rearing, and jumping in response to water stimulation. Water did produce a large increase in paw shaking compared to what was observed in $\mathrm{FVB} / \mathrm{NJ}$ mice. This could indicate that the DI water used as a stimulus could have a slightly bitter, aversive, or acidic taste to B6 mice that may not have been detected by FVB/NJ mice. This effect has also manifested in rats, where they generalized water to be "quinine-like" (Grobe \& Spector, 2008). Also, it is possible that even though the mice 
had undergone several sessions of water stimulation through the cannula in their mouth by the time they were tested, they may not have been acclimated to this new sensation during the delivery of tastants and their behaviors could have been influenced by the presence of the intra-oral cannula.

The response of B6 and ICR outbred mice to sucrose also produced similar behaviors, such as mouth movements and paw shaking. However, the ICR mice also produced a small amount of spitting in response to sucrose that was not seen in the B6 mice. The response to sucrose in $\mathrm{B} 6$ mice was also similar to that of $\mathrm{FVB} / \mathrm{NJ}$ mice, with two exceptions. B6 mice produced more paw shakes and mouth movements in response to sucrose than $\mathrm{FVB} / \mathrm{NJ}$ mice did. The increase in paw shakes could have been caused by having dissolved the sucrose in the same DI water that the mice were stimulated with in the water group. B6 mice are known as a strain that prefers and ingests sucrose, so this increase in mouth movements may mean that they were responding more to the sweet taste. In rats and hamsters, sucrose elicited only mouth movements (lateral tongue protrusions and tongue protrusions), which revealed a species difference between hamsters and rats from mice (Brining et al., 1991; Grill \& Norgren, 1978a). Differences in the taste reactivity procedures between studies may have also affected the differences found between the species. The lack of other behaviors being produced during testing may have been caused by the fact that the hamsters were only subjected to one 1-minute stimulation compared to the 20,15 second-stimulations the mice in our study were subjected to. There might be a time-dependent aspect to some of the behaviors that were found to be elicited by taste reactivity. In the other study, FVB mice were stimulated with 20 1-minute trials over a time course of 30 minutes. In this study, the B6 mice were stimulated with 20,15 seconds on/ 45 seconds off trials over a time course of 20 minutes. The decrease in time between the stimulation with taste stimuli may have also produced the differences found between the strains. On the other hand, a study performed in rats found the paw shaking and face grooming behaviors elicited by sucrose identical to mice in this study, but there are not enough details given in their article to be able to compare the procedure performed to ours (Kiefer \& Dopp, 1989).

The response of B6 mice to MPG was similar to that found in rats. Both species produced a high level of mouth movements and a low amount of paw shaking (Grill \& Flynn, 1987). This study investigated other umami compounds that were not utilized in this portion of the study, including MAG and MSG. They found that MSG produced the most ingestive taste behaviors and there was no difference between MPG and MAG. They also found that at higher concentrations, MPG produced more aversive behaviors, and at the lowest concentration, there was no difference between all three of the umami stimuli in aversive behaviors. Based on this, it is not surprising that both MPG and MPG+IMP elicited similar numbers of each of the kinds of behaviors. What was surprising is that based on the "synergism" found in the two-bottle tests performed in this study and previous electrophysiology data (Tokita \& Boughter, 2012; Tokita et al., 2012), I expected to observe an increase in hedonic taste reactivity behaviors in response to MPG+IMP, but the mixture did not seem to produce a measurable increase in intensity (as behavior) in this test. 
The taste reactivity test did not show much of a difference between the four preferred sweet and umami stimuli compared to each other or to water. This differs from what was found in rats, in which the number of hedonic behaviors following MSG and sucrose was found to be higher than following water. This difference could be attributed to many different factors. Firstly, this second study used MPG, which we found in our first study using two-bottle preference tests to be less preferred compared to MSG, which was used in the rat study. Secondly, this study had small group numbers, so an increase in animals may elucidate a difference that we are not currently seeing. Thirdly, in the mice in this study, it was only recorded whether or not a particular behavior occurred in a trial and in studies by Grill and Flynn, in rats, and Keifer et al., in mice, every time a behavior occurred it was recorded. In addition, in the studies by Kiefer et al. in mice and Grill and Flynn in rats, all of the behaviors were not separated out, instead, they are grouped together into two categories; either aversive or hedonic. Also, it is possible that the paws to mouth behavior, that the B6 mice in this study and the FVB/NJ mice in another study exhibited when drinking fluid, via an intra-oral cannula, could have blocked the view of the camera from being able to see the specific tongue movements that have been observed in rats (Travers et al., 2007). If for example, there is a knockout mouse that does not exhibit this paws to mouth behavior that is seen in B6 and FVB/NJ, this would make it possible to quantify these tongue movements and might hold the key to finding the differentiation between hedonic stimuli (Travers et al., 2007). Lastly, there may simply be a species difference in taste reactivity behavior in these stimuli between rats and mice.

By analysis of the taste reactivity data in a different manner, rather than just the percentage of trials a behavior occurred during the test, we found other differences and similarities between the stimuli. This analysis revealed that the majority of the behaviors occurred over the entire 20 -minute test. This representation of the stimuli revealed a difference in the appearance and consistency between the umami stimuli and the sweet stimuli in the behaviors of paw shaking, jumping, and rearing. It also highlighted differences between quinine and the other taste stimuli, with jumping and spitting occurring throughout the trial, and not occurring with the other taste stimuli. Also, quinine did not produce facial grooming like the other stimuli did. Lastly, it revealed that the sweet stimuli did not produce any jumps at all during the test, only intermittently produced rearing during the second half of the test, compared to consistent rearing with the other stimuli, and did not produce consistent paw shaking throughout the trials like the other stimuli did. This method of analysis shows that there could be more differences between the taste stimuli than may be seen with other methods of quantifying the data.

Characterizing taste reactivity in mice is possible, but much more challenging to obtain than what has previously been reported in rats. The smaller mouse makes it hard to distinguish the finer mouth movements (like tongue movements and gapes) that can be seen in rats, and instead, this study, behaviors were quantified that were easier to visualize (chin, paw, and mouth movements). In summary, this method could be used successfully to identify the behavioral characteristics of aversive stimuli in mice, but not to discriminate palatable stimuli from one another. 
In conclusion, relative to the initial hypothesis that sweet and umami stimuli would be similarly preferred and elicit matching orofacial behavioral patterns, the results suggested that the hypothesis was only partly correct. First, the sweet and umami stimuli were all highly preferred, especially MSG and MSG+IMP. These behavioral results reinforce that synergistic umami mixtures may be just as highly preferred as sucrose in B6 mice. This simple and high-throughput behavioral test revealed insights into the similarity of these compounds. It showed that mice both preferred them very strongly to water, but also exhibited a strong response to consuming them at a higher level than the other stimuli tested. Secondly, however, even though taste reactivity behaviors elicited by sweet (saccharin and sucrose) and umami (MPG+amil and MPG+IMP+amil) stimuli were found to not be significantly different from one another, they were also not significantly different from water. Also, looking at the percentage of trials that the sweet and umami stimuli elicit the nine different behaviors, the two umami stimuli are more similar to each other than the sweet stimuli. This did not support the portion of the hypothesis that sweet and umami compounds would elicit similar amounts of and similar taste reactivity behaviors. The only taste stimulus that was found to be significantly different from the others in quite a few behaviors (paw shakes, face grooming, and rearing) was $\mathrm{QHCl}$, showing that the taste reactivity screen predominantly revealed that bitter compounds elicited clearly unique behaviors. 


\section{CHAPTER 4. IMMUNOHISTOCHEMICAL VISUALIZATION AND 3D MAPPING OF C-FOS ELICITED FROM SWEET, BITTER, AND UMAMI STIMULI IN TASTE PORTIONS OF THE PBN IN C57BL/6J MICE}

This chapter covers the anatomical portion of the experiment that investigates Specific Aim 2- to create a map of neuronal activation in the parabrachial nucleus (PBN) in response to sweet- and umami-tasting compounds, (specifically sucrose and the synergistic mixture MPG+IMP+amil) to determine if the pattern of $c$-fos activation is similar in sweet and umami stimuli. The following analysis includes immunohistochemistry.

\section{Introduction}

\section{Inducible Proto-Oncogenes}

Proto-oncogenes are a class of oncogenes that serve the purpose of conveying information either within a cell or between two cells (Morgan \& Curran, 1991). These molecules can take many different forms, including extracellular polypeptide messengers, cell-surface receptors, protein kinases, G-proteins, and nuclear transcription factors (Morgan \& Curran, 1991). In neuroscience, one commonly used molecule in this group is the fos proto-oncogene, which was also later referred to as an intermediate early gene (Lau \& Nathans, 1987). The name fos was coined from the fact it was used to represent the "oncogene encoded by the Finkel-Biskis-Jinkins murine osteogenic sarcoma viruses" (Morgan \& Curran, 1991, pg. 423). Later, a cellular version homologous to the viral version was found in both the mouse and humans and was named c-fos (Curran, MacConnell, van Straaten, \& Verma, 1983). The $c-f o s$ gene is eventually translated to a $62 \mathrm{kDa}$ nuclear protein (Muller, Bravo, Burckhardt, \& Curran, 1984). C-fos is part of a family of other genes that contain leucine-zippers that are involved in transcription and can form dimers with either other members of the fos family or the jun family that also contain leucine-zippers (Herdegen \& Leah, 1998; Landschulz, Johnson, \& McKnight, 1988).

Fos has been found to be a general transcription factor that is induced short-term by a variety of environmental factors, such as taste (Montag-Sallaz \& Montag, 2006), stress (Armario, 2006), electrical stimulation (Morganti, Odegard, \& King, 2007), and analgesics as well as anesthetics (Armario, 2006; Bullitt, 1990). These stimuli are forms of new, increased, or unusual activation of neurons within the nervous system. These stimuli, in turn, produce the induction of $c$-fos protein and RNA relatively quickly (Morgan \& Curran, 1991). The $c$-fos transcriptional process begins at five minutes after the stimulus in the nucleus and continues to produce mRNA for a total of 15-20 minutes (Greenberg \& Ziff, 1984). This mRNA builds up inside the nucleus and is at the highest level between 30-45 minutes after the initial stimulation has first occurred and then quickly lowers back to the normal level (Morgan \& Curran, 1991; Muller et al., 1984). 
The $c$-fos protein is then produced and reaches its highest level approximately 2 hours following the initiation of the stimulus (Muller et al., 1984). The $c$-fos gene also starts a cascade that results in the eventual activation of a set of late genes (Herdegen \& Leah, 1998).

In brain tissue, $c$-fos and other members of the proto-oncogene family are often visualized within the nucleus of neurons by the use of immunohistochemistry (Terleph \& Tremere, 2006). In these experiments immunocytochemical localization of $c$-fos was utilized, since it is the most commonly used, has a commercially available antibody, and is accepted in the taste field. This technique employed $c$-fos immunocytochemistry to identify where taste neurons were located in the PBN and which neurons are activated in response to sweet, bitter, and umami stimuli in B6 mice. This was done in order to determine if there are unique neurons activated with each tastant or an overlap in the distribution of the activated neurons. After stimulation with a taste solution, the mouse brain begins activating neurons in the taste pathway, and in particular, neurons within the PBN are activated (Yamamoto et al., 1994) and starts a cascade of activity that ultimately produces depolarization, via calcium ions into the PBN and other neurons, which then activates the $c$-fos gene to ultimately produce the c-fos protein in the nucleus of those neurons (Morgan \& Curran, 1986). Using a $c$-fos antibody and performing immunohistochemistry, each neuronal cell that was stimulated and produced the $c-f o s$ protein can be stained and individually identified in the PBN (or other brain area being investigated). It is to be noted that in many of the cells in the CNS, except in the dentate gyrus and piriform cortex, there are normal, relatively low levels of both $c$-fos mRNA and protein expression (Morgan \& Curran, 1991). C-fos is thought to be produced in response to action potentials (Fields, Eshete, Dudek, Ozsrac, \& Stevens, 2001) and most likely represents "a monitor of intra-cellular second messenger levels" (Morgan \& Curran, 1991, pg. 440). This technique does not interfere with methods of stimulus delivery and enables examination of multiple areas of the brain at the same time point in response to the same stimulus (Mello, 2006).

This technique does come with a few limitations. In the past, $c$-fos antibodies had problems with specificity and also stained other members of the fos family (CastonBalderrama, Cameron, \& Hoffman, 1998; Van Der Gucht, Vandenbussche, Orban, Vandesande, \& Arckens, 2000). This problem has been successfully resolved with newer, more specific $c$-fos antibodies (Van Der Gucht et al., 2000), such as the one used in this study (c-fos, Santa Cruz Biotechnology, Santa Cruz, California). Stress and neophobia have also been found to elicit $c$-fos in mice (Montag-Sallaz, Welzl, Kuhl, Montag, \& Schachner, 1999). These two issues can be carefully controlled for, by having the mice handled by humans and letting them acclimate to both the testing chamber and the testing procedure before the actual testing begins. Also, $c$-fos is only transcribed in response to excitatory action potentials in the brain [Krukoff, (n.d)]. Therefore, it may be possible that we will not be mapping the entire response to the taste stimuli, if it results in inhibition [Krukoff, (n.d)]. However, many previous studies have used this technique to map neuronal responses to taste compounds and have shown that it can be used successfully (Chan, Yoo, \& Travers, 2004; Haino et al., 2010; Travers \& Hu, 2000; Yamamoto \& Sawa, 2000; Yamamoto et al., 1994; Yamamoto et al., 1993). Lastly, 
quantifying $c$-fos is a post-mortem technique and cannot be reproduced in the exact same animal. The general taste responses of each genetically identical animal to each stimulus should be similar enough as has been found in Chapter 3, that an average of the $c$-fos expression should be able to be characterized to the different taste stimuli.

\section{Taste and $c$-fos}

Previous studies have used $c$-fos as a marker of taste-evoked activity and have identified some specific regions of the PBN subnuclei in rats, in terms of taste quality representation. For example, neurons that responded to salt taste were found in the rat to be located in the CMS subnucleus (Yamamoto et al., 1994; Yamamoto et al., 1993; Yamamoto et al., 2009). The DLS is possibly involved in the consumption of preferred and familiar substances (Yamamoto et al., 1994; Yamamoto et al., 2009). Aversive compounds, such as quinine, activated neurons in the caudal portion of the EMS and ELS (Travers et al., 1999; Yamamoto et al., 1994; Yamamoto et al., 1993). Neurons located in the rostral ELS seem to be predominantly involved with gastrointestinal visceral stimuli (Yamamoto et al., 1994). Both anatomical and electrophysiological evidence have suggested that palatable taste information, including sweet taste, has been principally found in the medial PBN and waist area in rats and mice (de Araujo, 2009; Halsell \& Travers, 1997; Tokita \& Boughter, 2016; Yamamoto et al., 1994). On the other hand, aversive taste information and visceral information (along with cardiovascular, respiratory, and other gastrointestinal information) was primarily found in the lateral PBN (de Araujo, 2009; Yamamoto et al., 1994).

\section{Materials and Methods}

\section{Animals}

Of the original 46 animals tested, a total of 35 male and female mice were used for analysis in these anatomical studies. The omission of these 11 mice was due to technical issues with perfusion or histology. Prior to intra-oral cannula surgery, mice were group-housed based on sex in standard plastic mouse cages $(28 \times 17.5 \times 13 \mathrm{~cm})$ with free access to normal mouse chow and water. After the surgery, mice were individually housed. The evening prior to taste reactivity testing, mice were food-deprived but continued to have free access to water. Animals were treated according to a protocol approved by the University of Tennessee Health Science Center Institutional Animal Care and Use Committee. There were 6 groups of animals, one for each taste stimulus tested. For tissue in which the PBN was analyzed the groups were $\mathrm{QHCl}(\mathrm{n}=7)$, saccharin $(n=5), M P G+\operatorname{IMP}(n=6), \operatorname{MPG}(n=6)$, and a DI water group $(n=4)$. I used the tissue from one mouse in each group to create a three-dimensional model using Neurolucida software. This tissue was selected because it was the most complete and most evenly stained set of tissue at the time, in each group. In the same 6 groups of animals, $c$-fos expression was also analyzed in the locus coeruleus (LC) and mesencephalic nucleus of 
the trigeminal nerve (Me5) in 35 mice, except for one less mouse in the MPG group and one more mouse in the saccharin group. $C$-fos stained nuclei were counted in these nongustatory areas (that are located at the same rostral-caudal level as the PBN) as a control for mouse-to-mouse variability in background $c$-fos expression.

Both males and females $(\mathrm{H} 20=3$ males and 1 female, $\mathrm{QHCl}=6$ males and 1 female, $\mathrm{SUC}=2$ males and 5 females, $\mathrm{SAC}=3$ males and 2 females, $\mathrm{MPG}+$ amil $=2$ males and 4 females, MPG + IMP + amil $=2$ males and 4 females) were used for these experiments and were either bred in the animal colony or purchased from the Jackson Laboratory (Bar Harbor, ME). Prior to testing, animals were group housed and separated according to sex in standard plastic mouse cages $(28 \times 17.5 \times 13 \mathrm{~cm})$ with ad libitum access to normal dry pellet chow and water. At the time of testing, animals were approximately 3-5 months old.

\section{Testing Procedures}

\section{Solutions}

Solutions used for experiments in Aim 2 include $0.01 \mathrm{M}$ sodium saccharin, $1 \mathrm{M}$ sucrose, $0.003 \mathrm{M} \mathrm{QHCl}, 0.1 \mathrm{M}$ MPG+10 $\mu \mathrm{M}$ amil, $0.1 \mathrm{M} \mathrm{MPG}+0.01 \mathrm{M} \mathrm{IMP}+10 \mu \mathrm{M}$ amil, and DI water. The solutions were prepared either the day before or the day of testing. Amiloride was added to the umami solutions to prevent binding to epithelial sodium channels in the taste buds.

\section{Perfusions and Sectioning}

Immediately after the 20-minute taste reactivity testing session, each mouse was returned to their home cages, and the water was removed. Each mouse was anesthetized 2 hours after the beginning of its testing session with an intraperitoneal injection of $25 \%$ urethane and transcardially perfused with $0.1 \mathrm{M}$ PBS followed by, fixative. The brain was removed, cryoprotected with a $20 \%$ sucrose $/ 10 \%$ glycerol $/ 0.1 \mathrm{M}$ PB solution, and frozen sectioned, and immunohistochemically stained according to previously described methods (Chapter 2) and in Table 4-1. Sections were processed as free-floating at room temperature unless otherwise stated. Every other section in the area of the PBN was utilized for $c$-fos immunohistochemistry, which was then performed on it; the other sections were stained with cresyl violet to serve as morphological references. Control sections were processed for immunohistochemistry at the same time with the omission of the primary $c$-fos antibody. Figure 4-1 depicts images of brain sections illustrating $c$-fos staining (Figure 4-1A and B) and in the absence of primary antibody (Figure 4-1C and D), which showed no $c$-fos positive staining. 
Table 4-1. Immunohistochemistry Steps Used for Labeling $c$-fos.

\begin{tabular}{ll}
\hline Histology Step & Time \\
\hline Following perfusions, brains placed in 10\% formalin, stored at $4{ }^{\circ} \mathrm{C}$ & $1-3$ days \\
Brains placed in $20 \%$ sucrose/10\% glycerol solution & $24-48 \mathrm{hrs}$ \\
Sectioning on a sliding microtome at 40 micrometers & N/A \\
Floating sections placed in 0.1 M PB & N/A \\
Wash: 0.1 M PB, 3x & 5 min each \\
Quenching: 30\% H2O2 & 30 min \\
Wash: 0.1 M PB, 3x & 5 min each \\
Blocking and Primary: Normal goat serum, 0.8\% PBTx, and primary & Overnight \\
antibody, c-fos & \\
Wash: 0.1 M PB, 3x & 5 min each \\
Secondary: Donkey anti-rabbit w/ 0.8\% PBTx & $1 \mathrm{hr}$ \\
Wash: 0.1 M PB, 3x & $5 \mathrm{~min}$ each \\
PAP: Placed in Rabbit Peroxidase-Anti Peroxidase solution w/ 0.8\% & $1 \mathrm{hr}$ \\
PBTX & \\
Wash: 0.1 M PB, 3x & $5 \mathrm{~min}$ each \\
Ni-DAB: Nickel Diaminobenzidine & $10 \mathrm{~min}$ \\
+ H2O2 & $10 \mathrm{~min}$ \\
Wash: 0.1 M PB, 3x & $5 \mathrm{~min}$ \\
Mount, dry, and coverslip & $\mathrm{N} / \mathrm{A}$ \\
\hline
\end{tabular}



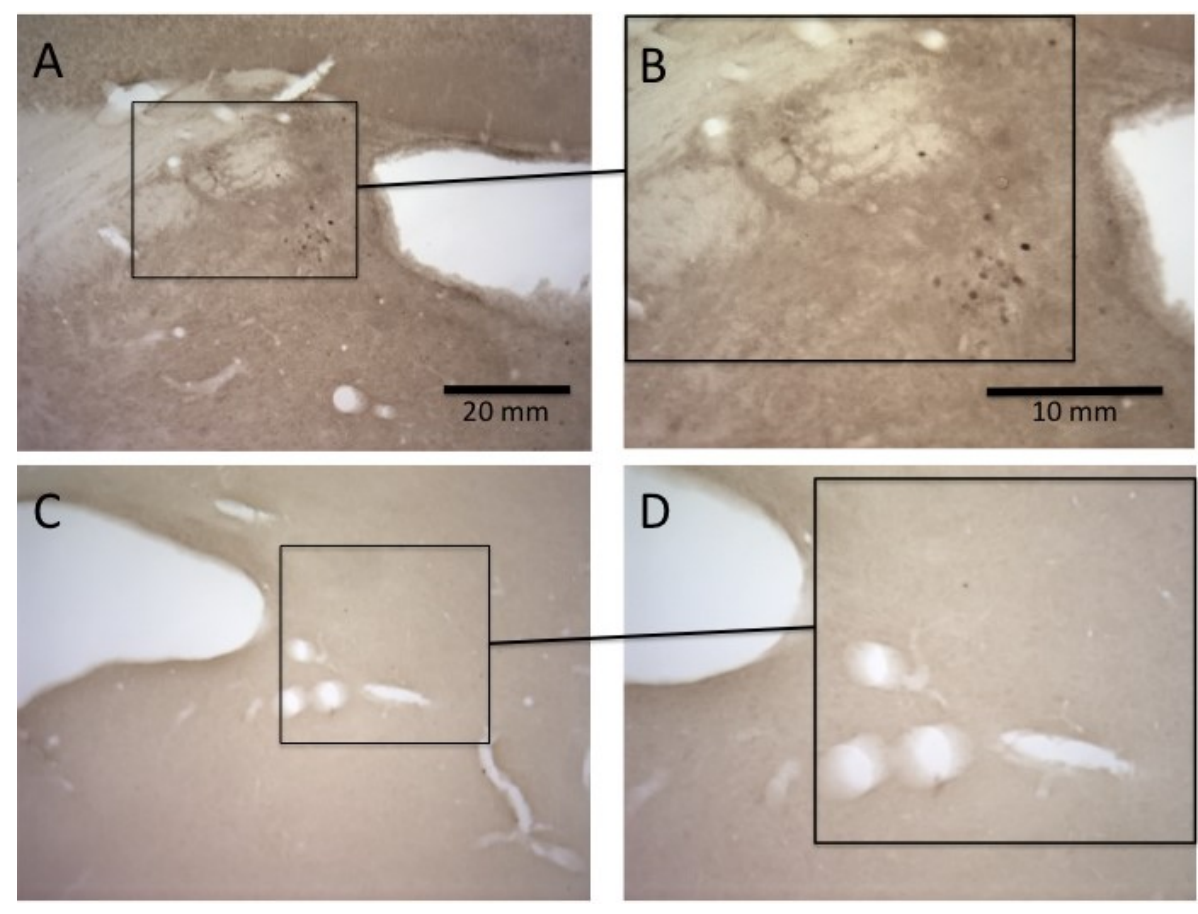

Figure 4-1. Primary Antibody Control.

Notes: A) 10x image of a brain section from a QHCl-stimulated mouse stained for $c$-fos. B) 20x image of the same brain section as seen in (A) in the area inside the black box to show better detail of the $c$-fos staining. C) 10x image from a brain slice from the same QHCl-stimulated animal in a more caudal brain region that was processed for $c-f o s$ at the same time, but without the primary antibody. D) 20x image from the same brain slice as seen in $\mathrm{C}$ from the area inside the black box so you can see the lack of $c$-fos staining since it is missing the primary antibody. 


\section{Analysis}

\section{Three-Dimensional Reconstruction}

Three-dimensional reconstruction and analysis of c-fos patterns throughout the entire gustatory portion of the PBN were traced with video guidance using Neurolucida software (MBF Biosciences Inc., Colchester, VT) with the use of a 20x lens. This system is mounted on a Nikon Optiphot microscope. One animal per group was analyzed in this manner, both the superior cerebellar peduncle and a circular area surrounding the PBN subnuclei were traced in every other section in order (DAB-stained sections). These were used as landmarks to help align subsequent sections. In the DAB-stained sections, the $c$ fos reactive neurons were identified and marked using the software. The Neurolucida software was then used to create the three-dimensional reconstructions of the tissue for the left portion of the PBN. A three-dimensional model of the superior cerebellar peduncle was also created using the Paxinos Mouse brain atlas and Blender software and used as a model for the 3-dimensional reconstruction of the PBN with actual tissue sections.

\section{C-fos Counts}

After mounting prepared tissue slices onto slides and cover-slipping them, DABlabeled $c$-fos in all sections were imaged using the 10x lens of a Leica (DMRXA2, Leica Microsystems, Bannockburn, IL, USA) episcopic-fluorescence microscope equipped with a digital camera Hamamatsu ORCA-ER (Hamamatsu Photonics, Shizuoka, Japan) and imaging software (SimplePCI, Compix Inc., Cranberry Township, PA, USA). The images of all of the sections, that included all of the taste relevant portions of the PBN, (DABlabeled and cresyl violet stained) for each animal were put in order from caudal to rostral; this included all of the taste relevant portions of the PBN. Then the images were given to the PI (J.D.B) who assigned each animal a case number, to assist in a "blind" analysis of the sections as to what tastant the mouse has been exposed to. The outlines of the PBN subnuclei (dorsal medial, medial, ventral lateral, waist area (portion of the brachium conjunctivum that spanned between the medial and the ventral lateral subnuclei), dorsal lateral, central lateral, external lateral, and external medial) were traced blindly in the tissue from 35 animals using the software Image J. The outlines of the LC and Me5 were also traced at a later time in the tissue from 35 animals using the GNU Manipulation Program (GIMP). The immunolabeled $c$-fos positive nuclei were counted blindly, using separate markers for each subnucleus throughout each section and continuing through the entire PBN (5-7 sections per mouse) of each brain and recorded. The immunolabeled $c$ fos positive nuclei in the entire LC and Me5 were also counted using separate markers for each area (6-8 sections per mouse) of each brain and recorded.

\section{Statistics}

Left and Right Portions of Subnuclei/Nuclei. The number of $c$-fos positive nuclei in the two sides (left and right) of each PBN subnucleus, LC, and Me5 were compared to each other using either paired t-tests or Wilcoxon Signed Rank Tests. When using the 
paired t-test, there is the assumption that the data is normally distributed. The normality was tested and if it failed, the non-parametric equivalent, Wilcoxon signed rank test was used instead, which does not make that assumption.

Entire PBN, LC, and Me5. A one-way ANOVA was performed to see if there was a difference between the total number of $c$-fos positive nuclei counted in the PBN response to each of the taste stimuli. Kruskal-Wallis tests were performed on the total number of $c$-fos positive nuclei counted in the LC and the Me5.

Rostral, Intermediate, and Caudal Level PBN Subnuclei. Either one-way ANOVAs or Kruskal-Wallis Tests followed by either Bonferroni or Dunn's post-hoc tests, respectively on the number of $c$-fos positive nuclei in each of the eight subnuclei in these three levels. When using the ANOVA statistical test, the following two factors are assumed: that the data are normally distributed and the variances for each group are equal. In order to determine if these were indeed true, they were tested. If either one of these tests failed, the non-parametric equivalent, Kruskal-Wallis one-way analysis on ranks, was used instead, which does not make the same assumptions.

\section{Results}

\section{Representation of Tissue}

Every other slice of the tissue containing the PBN was processed using immunohistochemistry to stain and visualize the neuronal marker $c$-fos. The remaining slices were stained with cresyl violet to visualize the anatomy of the PBN. The tissue was mounted onto slides, coverslipped, imaged, and organized into rostral-caudal order. Sections containing the PBN were subsequently delineated into eight different PBN subnuclei using Image $\mathrm{J}$ software. The tissue was then renamed and blindly counted. A representative example of cresyl-violet and $c$-fos stained tissue indicating where the $c$-fos positive nuclei were located on the immunohistochemically stained sections from an individual mouse is shown in Figure 4-2. This example illustrates the delineation and position of the subnuclei across four representative levels of the PBN. $C$-fos positive nuclei were counted throughout the entire taste portion of the PBN including each of these eight subnuclei, without regard to the intensity of the staining, in tissue from animals stimulated with one of each of the six different taste stimuli. Representative plots showing taste-elicited $c$-fos positive nuclei expressed within the intermediate level of the PBN from corresponding sections for each of the six taste stimuli are shown in

Figure 4-3. At this one level, the sweet and MPG+IMP stimuli seem to exhibit a similar pattern of $c$-fos positive nuclei in the PBN. The $c$-fos staining pattern appears more intense, after QHCl-stimulation when compared to the other immunohistochemical staining of $c$-fos, although all were processed the same. (Figure 4-3). 

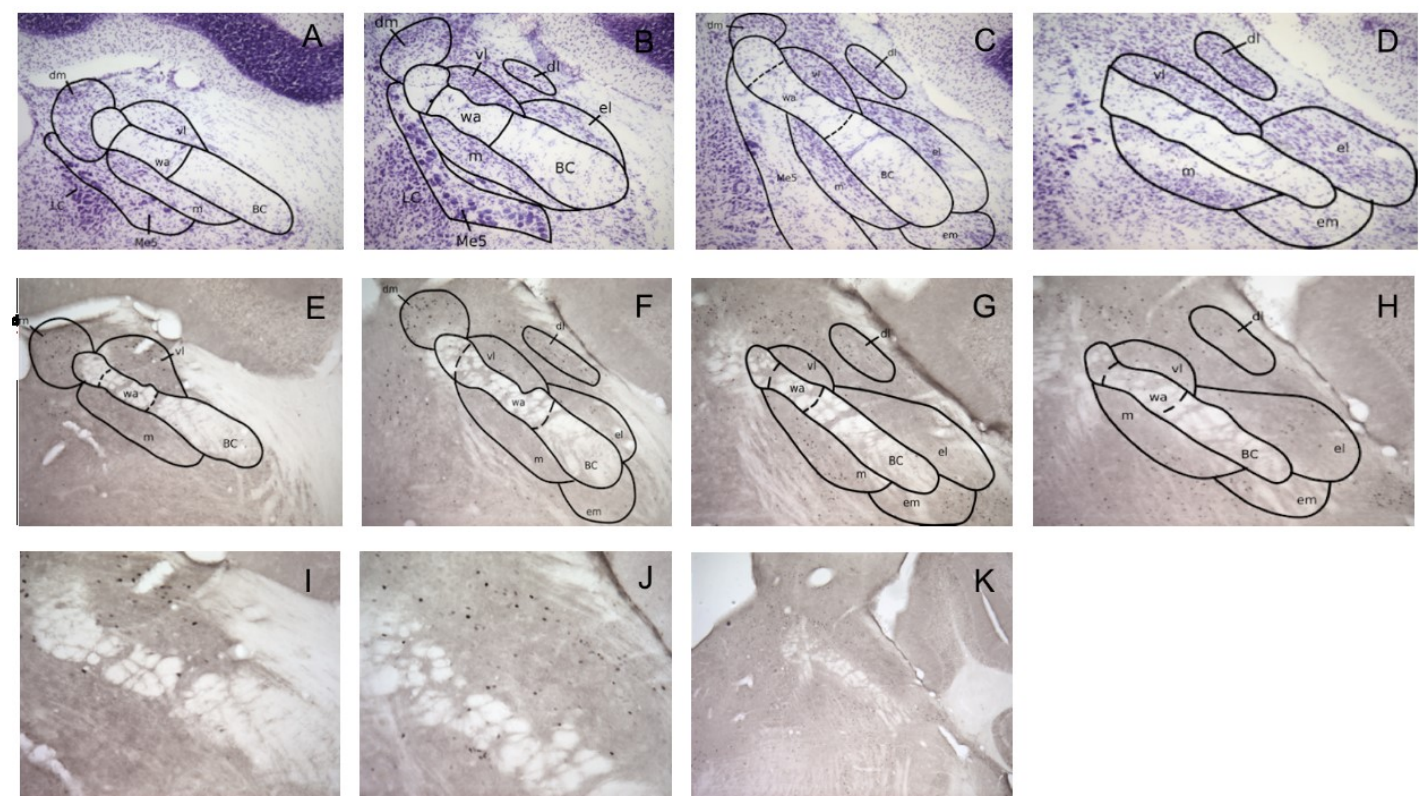

Figure 4-2. Tissue from a QHCl-stimulated Animal.

Notes: Representative sections from 4 levels of the right PBN from one single B6 mouse following taste reactivity stimulation with $\mathrm{QHCl}$. Cresyl violet stained sections are on the top row from caudal (A), intermediate (B) and (C), and rostral (D) levels of the PBN at $10 \mathrm{x}$ magnification. In the second row, underneath are the corresponding $c$-fos stained sections to the cresyl violet stained sections above from caudal (E), intermediate (F) and $(\mathrm{G})$, and rostral $(\mathrm{H})$ levels of the PBN at 10x magnification. (I) is a 20x magnification image of the $c$-fos stained waist of the SCP and the VL and M PBN subnuclei to more clearly see the $c$-fos positive nuclei. (J) is a 20x magnification image of the $c$-fos stained waist of the SCP, VL, and DL PBN subnuclei to more clearly see the $c$-fos positive nuclei. $(\mathrm{K})$ is a $c-f o s$ stained $5 \mathrm{x}$ magnification image of the entire SCP and rostral PBN subnuclei. (wa $=$ waist; $\mathrm{vl}=$ ventral lateral; $\mathrm{m}=$ medial, $\mathrm{dl}=$ dorsal lateral; $\mathrm{BC}=$ brachium conjunctivum; el = external lateral; em = external medial; Me $5=$ mesencephalic nucleus of the trigeminal nerve). 

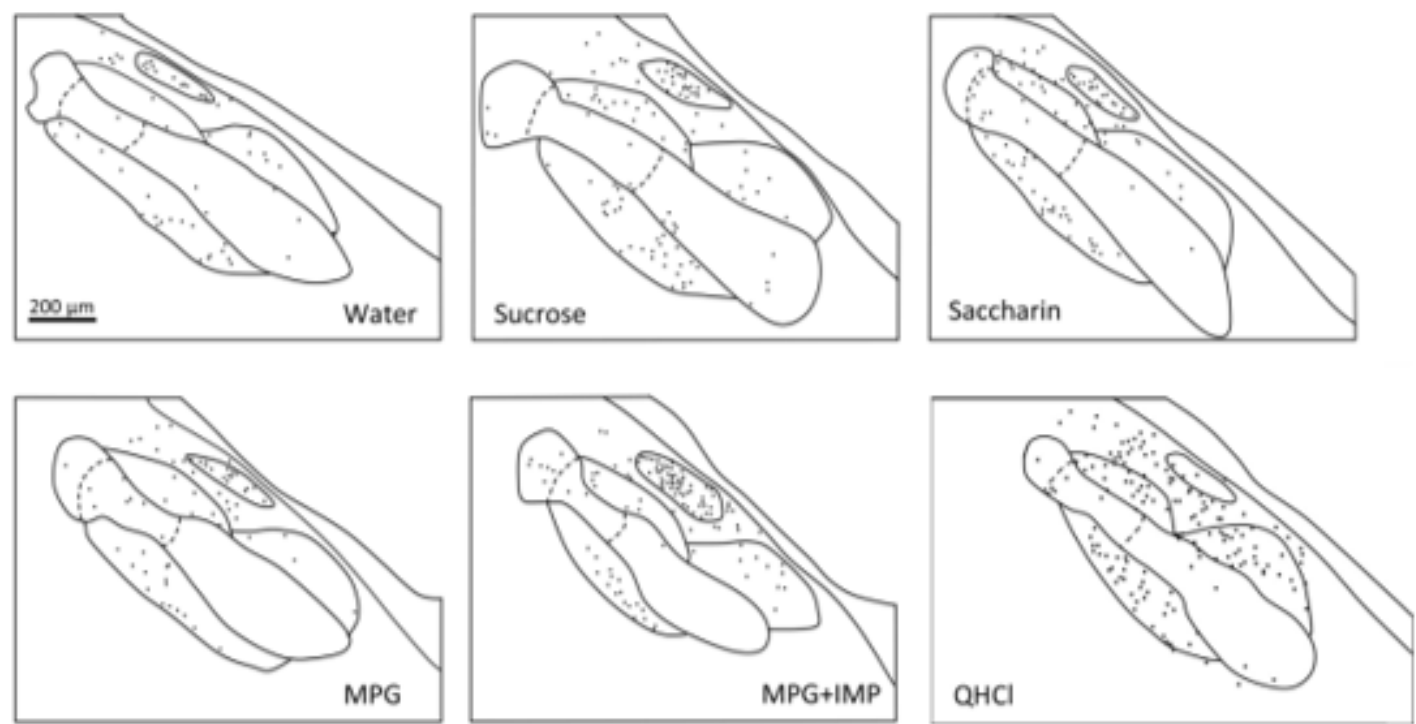

Figure 4-3. Diagrams of Delineated Subnuclei and Immunolabeled $C$-fos Created from Tissue from Animals Stimulated with DI Water, Sucrose, Saccharin, MPG, MPG+IMP, and Quinine Hydrochloride.

Notes: These diagrams were created from one section from individual mice from the intermediate level of the PBN. The water stimulated animal exhibits less $c$-fos overall in all of the PBN subnuclei. The sucrose, saccharin, and MPG+IMP stimuli seem to exhibit a similar $c$-fos pattern in the $\mathrm{PBN}$. The $\mathrm{QHCl}$ stimulated animal exhibits a more intense $c$ fos staining pattern overall. 


\section{Three-Dimensional Reconstruction}

The tissue regions from the taste portion of the left side of the PBN, from individual mice, stimulated with one of each of the six taste stimuli, were traced and formatted into a three-dimensional reconstructional image using Neurolucida software to look at the overall expression pattern in the PBN across space (Figures 4-4 through 4-9). All of these figures are from the left PBN, but some appear to be rotated because the slices were put on the slides backward. This method of portraying the stained $c$-fos positive nuclei illustrated the anatomical variation in the tissue itself, between the individual mice. Also, quite a few differences can be observed in the patterns of $c$-fos positive nuclei located within this tissue. The water-stimulated animal (Figure 4-4) appeared to possess less $c$-fos positive nuclei than the animals given other stimuli (Figures 4-5 through 4-9), even though that was not actually the case as seen in Table 4-2. The sweet stimuli (sucrose, saccharin; Figures 4-5 and 4-6) and the umami stimuli (MPG, MPG+IMP; Figures 4-7 and 4-8) evoked a similar pattern in overall $c$-fos expression in the PBN, but the 3D illustration highlighted subtle differences among them, including differing intensities of $c$-fos staining in the DM and $\mathrm{M}$ subnuclei. In terms of the umami stimuli, the $c$-fos expression pattern in the MPG+IMP-stimulated animal (Figure 4-8) was comparable to the MPG-stimulated animal (Figure 4-7), despite the fact that the MPG+IMP tastant was preferred to a greater extent. The 3D illustration also highlighted the fact that $\mathrm{QHCl}$ (Figure 4-9), elicited the most $c$-fos positive nuclei overall, as was suggested with the intensity observed in the single sections, additionally, the $c$-fos positive nuclei are clustered closer together and seemed to encompass the entire field, instead of being isolated to only particular subnuclei.

The Neurolucida program generated data from these 3D images, such as actual dimensions of the PBN and SCP traced, the number of $c$-fos positive nuclei counted, and the actual distances between the $c$-fos positive nuclei counted in the reconstruction from each mouse that was an example of the six stimuli (Table 4-2). These values, in agreement with the images, demonstrated the variation of the tissue in each individual mouse. The data showed that even though the amount of $c$-fos positive nuclei activated by water appeared to be the least amount among the stimuli, there was actually less $c$-fos positive nuclei in the saccharin-stimulated animal, in which there was the least amount of $c$-fos positive nuclei counted. The other noticeable difference is that the pattern of waterinduced $c$-fos positive nuclei was less clustered in both the SCP and the area of the PBN subnuclei.

\section{C-fos Counts}

C-fos expression data was subsequently quantified and analyzed in all mice using two-dimensional images. The number of $c$-fos positive nuclei in the two sides (left and right) of each PBN subnucleus were compared to each other using either paired t-tests or Wilcoxon Signed Rank Tests. This was to determine if the side that the intra-oral cannula was located on might have had an effect on the laterality of the PBN $c$-fos staining characteristics. There were statistically significant differences found in only 5 out of the 


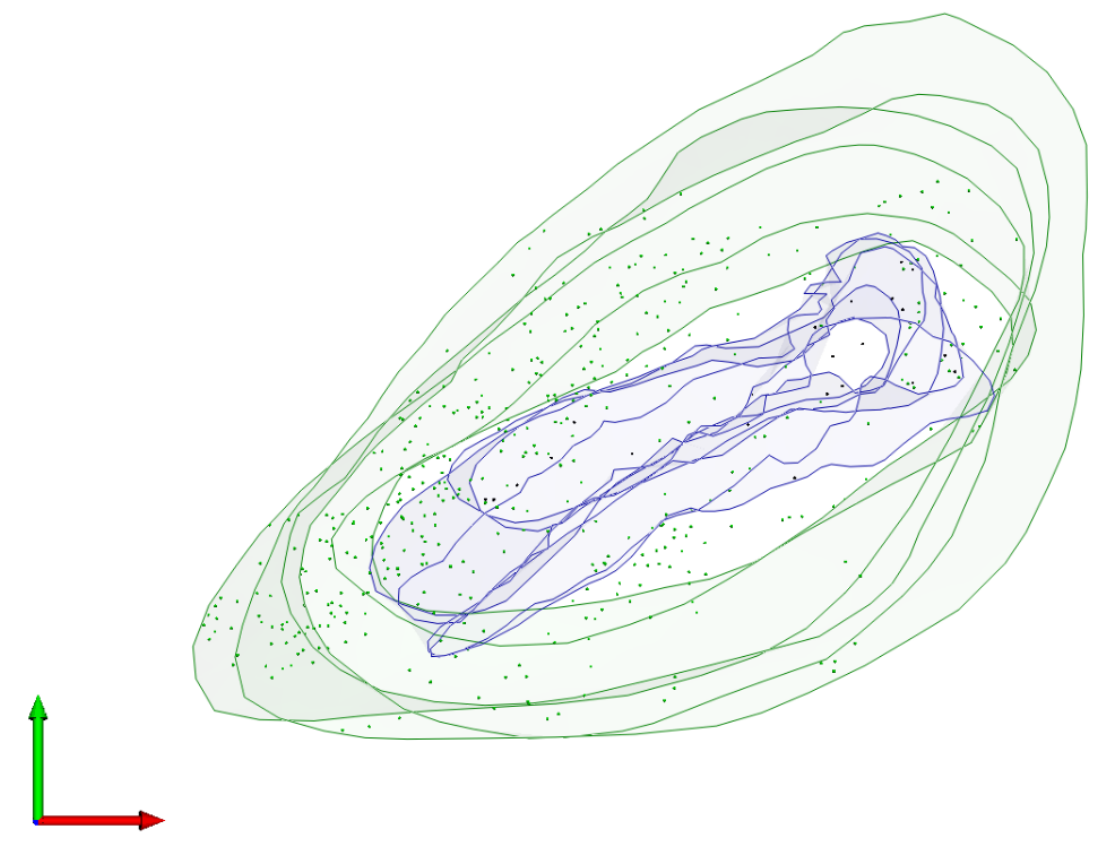

Figure 4-4. Three-Dimensional Reconstruction of the Left SCP and the PBN from a Water-Stimulated Animal.

Notes: Blue lines denote the SCP along with the blue $c$-fos marked inside of it. The green lines surround the PBN and contain green $c$-fos marked within. The red arrow illustrates $\mathrm{x}$, and the green arrow illustrates $\mathrm{y}$. 


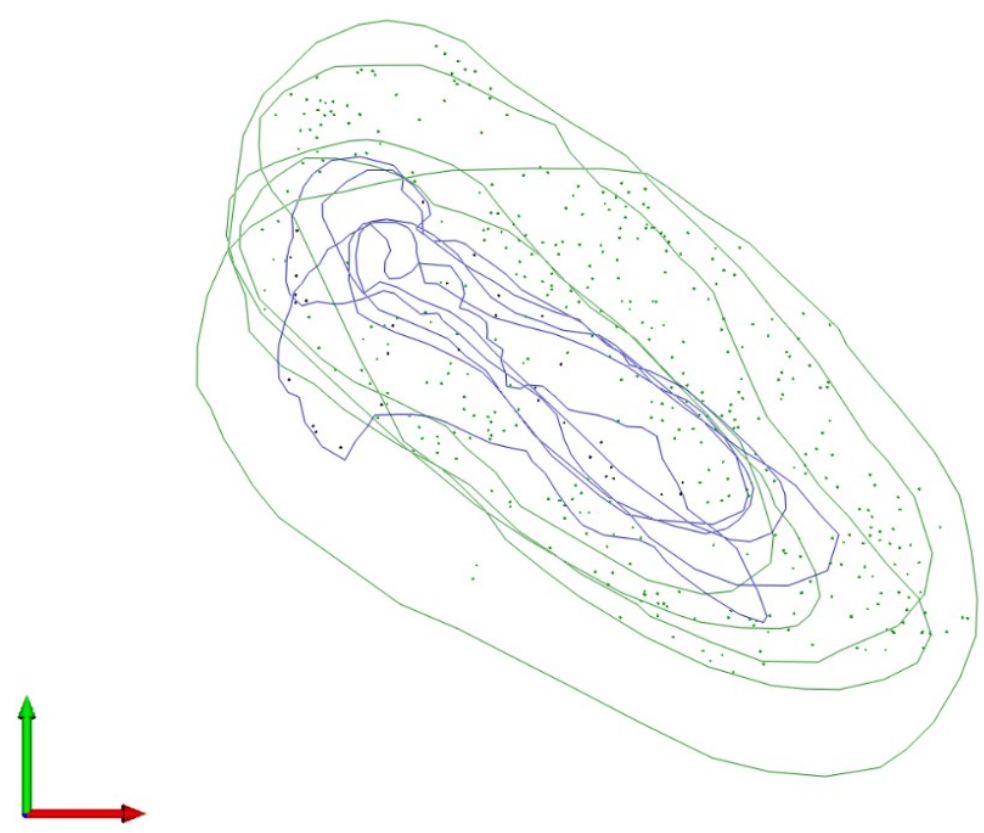

Figure 4-5. Three-Dimensional Reconstruction of the Left SCP and the PBN from a Sucrose-Stimulated Animal.

Notes: Blue lines denote the SCP along with the blue $c$-fos marked inside of it. The green lines surround the PBN and contain green $c$-fos marked within. The red arrow illustrates $\mathrm{x}$, and the green arrow illustrates $\mathrm{y}$. 


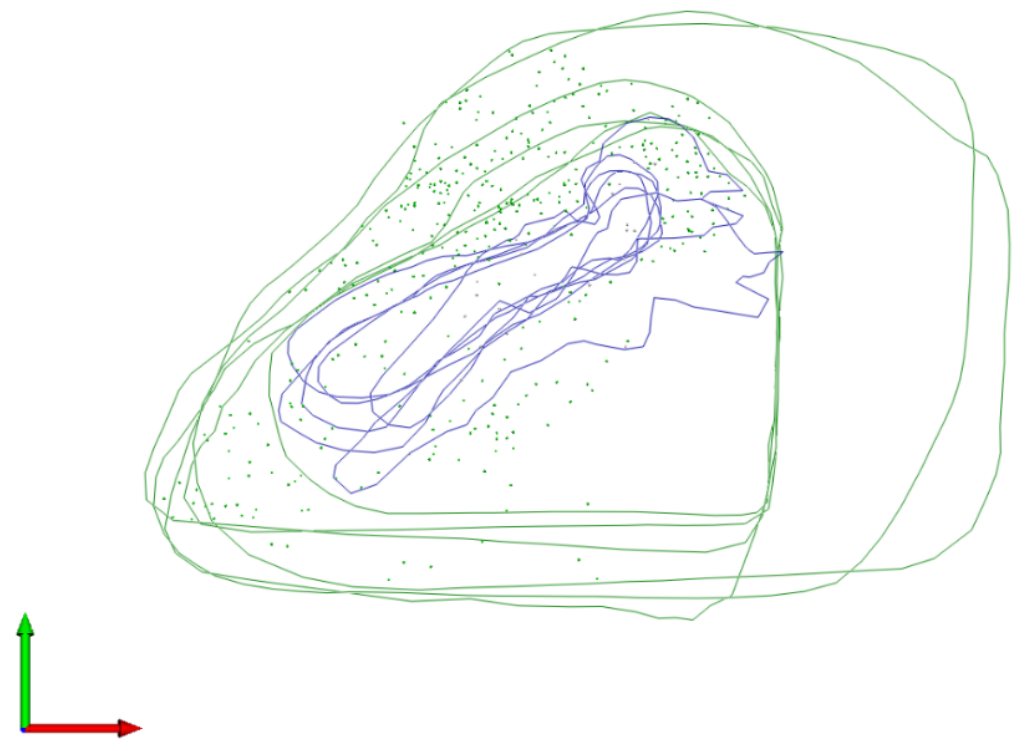

Figure 4-6. Three-Dimensional Reconstruction of the Left SCP and the PBN from a Saccharin-Stimulated Animal.

Notes: Blue lines denote the SCP along with the blue $c$-fos marked inside of it. The green lines surround the PBN and contain green $c$-fos marked within. The red arrow illustrates $\mathrm{x}$, and the green arrow illustrates $\mathrm{y}$. 


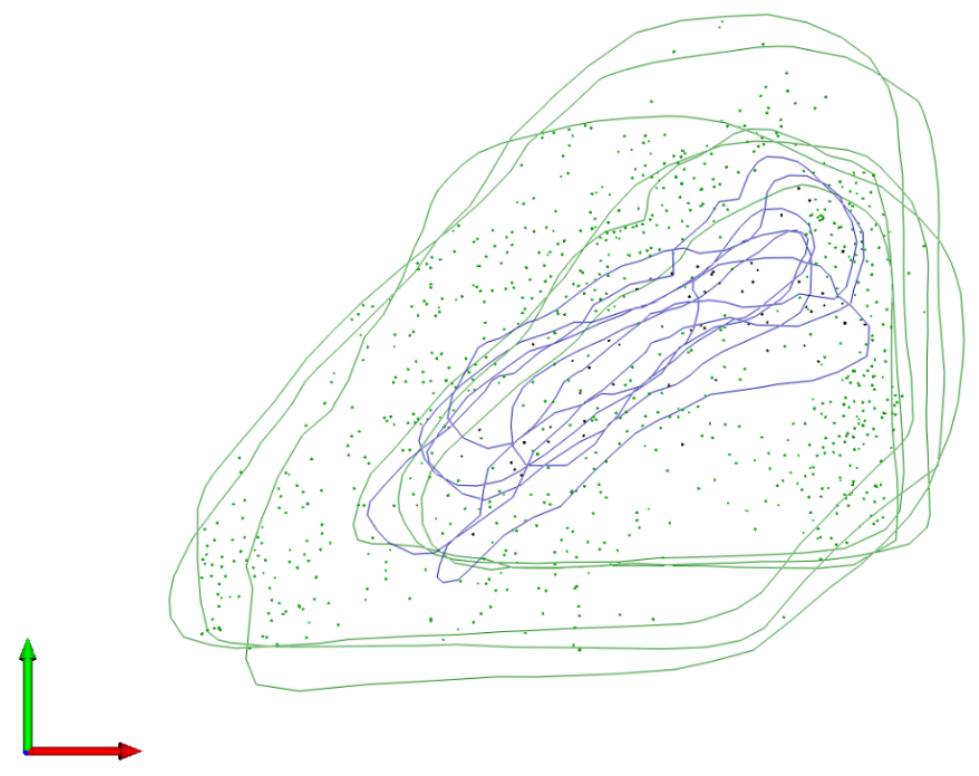

Figure 4-7. Three-Dimensional Reconstruction of the Left SCP and the PBN from an MPG-Stimulated Animal.

Notes: Blue lines denote the SCP along with the blue $c$-fos marked inside of it. The green lines surround the PBN and contain green $c$-fos marked within. The red arrow illustrates $\mathrm{x}$, and the green arrow illustrates $\mathrm{y}$. 


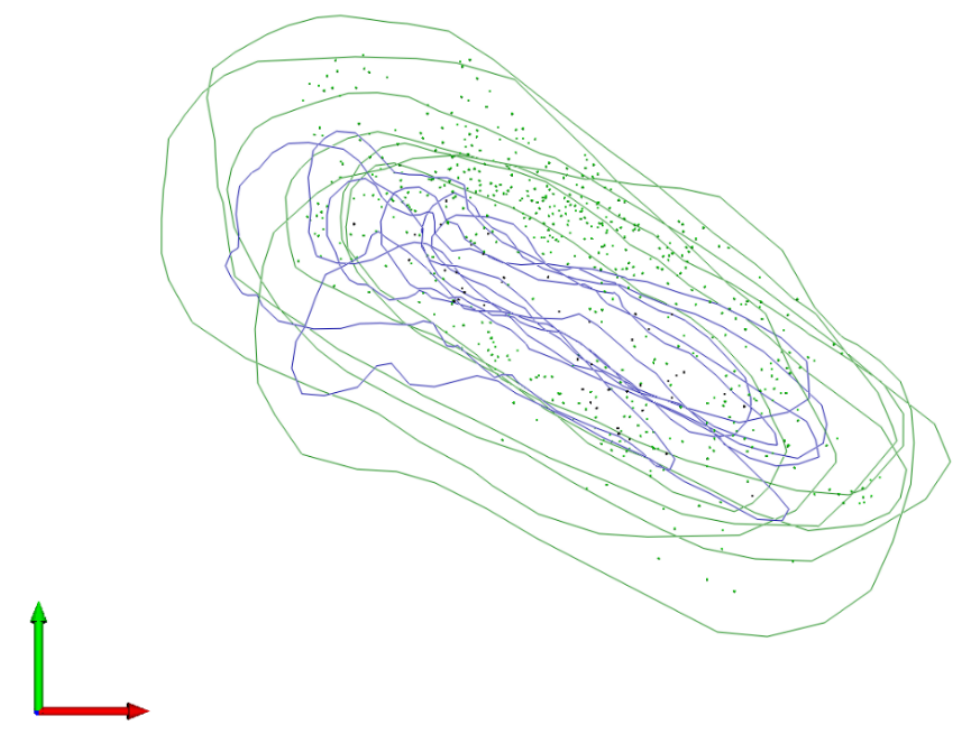

Figure 4-8. Three-Dimensional Reconstruction of the Left SCP and the PBN from a MPG+IMP-Stimulated Animal.

Notes: Blue lines denote the SCP along with the blue $c$-fos marked inside of it. The green lines surround the PBN and contain green $c$-fos marked within. The red arrow illustrates $\mathrm{x}$, and the green arrow illustrates $\mathrm{y}$. 


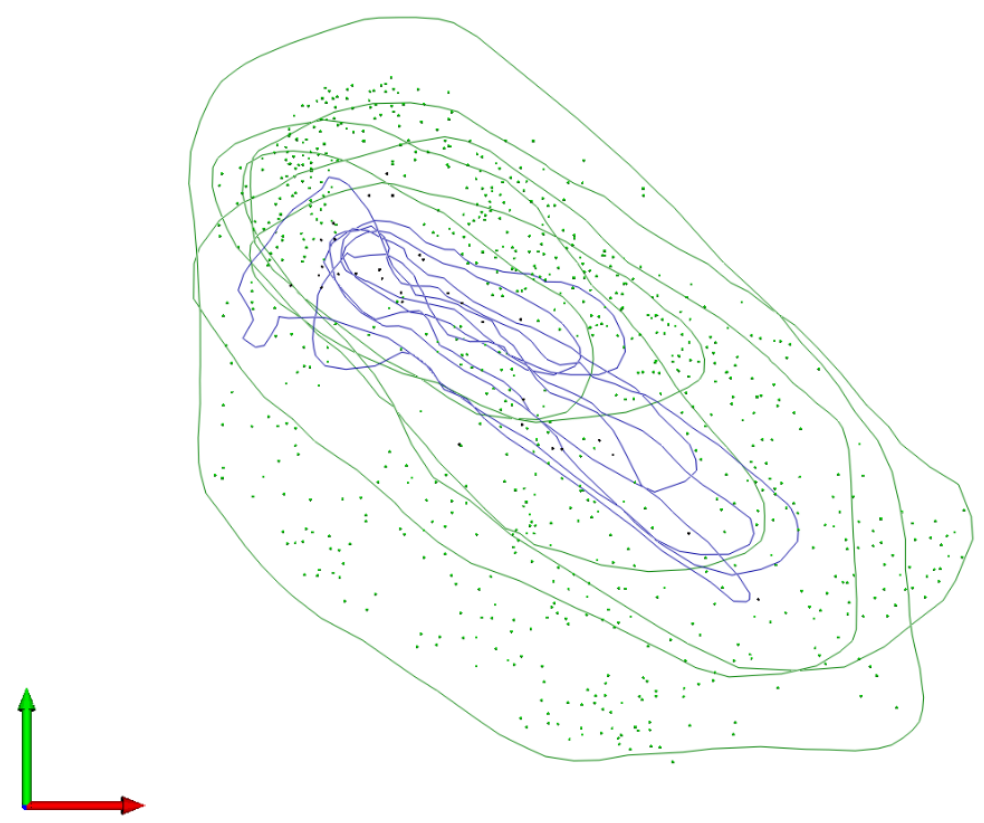

Figure 4-9. Three-Dimensional Reconstruction of the Left SCP and the PBN from a Quinine-Stimulated Animal.

Notes: Blue lines denote the SCP along with the blue $c$-fos marked inside of it. The green lines surround the PBN and contain green $c$-fos marked within. The red arrow illustrates $\mathrm{x}$, and the green arrow illustrates $\mathrm{y}$. 
Table 4-2. Three-Dimensional Reconstruction Neurolucida Data.

\begin{tabular}{|c|c|c|c|c|c|c|c|c|c|c|c|c|}
\hline $\begin{array}{l}\text { Taste } \\
\text { Stimulus }\end{array}$ & $\begin{array}{l}\text { Sections } \\
\text { counted }\end{array}$ & $\begin{array}{l}\text { Enclosed } \\
\text { volume of } \\
\text { SCP }(\mu \mathrm{m})\end{array}$ & $\begin{array}{l}\text { Surface area } \\
\text { of SCP }(\mu \mathrm{m})\end{array}$ & $\begin{array}{l}\text { Enclosed } \\
\text { volume of } \\
\text { PBN }(\mu \mathrm{m})\end{array}$ & $\begin{array}{l}\text { Surface area } \\
\text { of PBN }(\mu \mathrm{m})\end{array}$ & $\begin{array}{l}\text { Total \# } \\
\text { of } c \text {-fos } \\
\text { marked }\end{array}$ & $\begin{array}{l}\text { Ave. } \\
\text { Distance } \\
\text { of } c \text {-fos } \\
\text { in SCP } \\
(\mu \mathrm{m})\end{array}$ & $\begin{array}{l}\text { Ave. } \\
\text { Distance } \\
\text { of } c-f o s \\
\text { in PBN } \\
(\mu \mathrm{m})\end{array}$ & 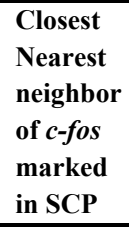 & 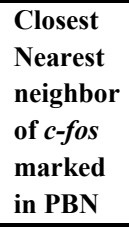 & $\begin{array}{l}\text { Farthest } \\
\text { nearest } \\
\text { neighbor } \\
\text { of } c-f o s \\
\text { marked } \\
\text { in SCP } \\
\end{array}$ & $\begin{array}{l}\text { Farthest } \\
\text { nearest } \\
\text { neighbor } \\
\text { of } c \text {-fos } \\
\text { marked } \\
\text { in PBN } \\
\end{array}$ \\
\hline Water & 6 & $1.75 \mathrm{E}+07$ & $5.46 \mathrm{E}+05$ & $1.00 \mathrm{E}+08$ & $1.85 \mathrm{E}+06$ & 450 & 63.1 & 29.9 & 21.7 & 6.9 & 140.5 & 91.3 \\
\hline Quinine & 6 & $1.30 \mathrm{E}+07$ & $4.14 \mathrm{E}+05$ & $7.25 \mathrm{E}+07$ & $1.63 \mathrm{E}+06$ & 1365 & 35 & 19.5 & 6.2 & 5.4 & 160 & 69.4 \\
\hline Sucrose & 5 & $1.80 \mathrm{E}+07$ & $5.63 \mathrm{E}+05$ & $7.22 \mathrm{E}+07$ & $1.66 \mathrm{E}+06$ & 430 & 50.7 & 27.1 & 11.3 & 5.9 & 134.1 & 108 \\
\hline Saccharin & 6 & $1.54 \mathrm{E}+07$ & $4.80 \mathrm{E}+05$ & $1.22 \mathrm{E}+08$ & $2.19 \mathrm{E}+06$ & 398 & 53.6 & 27.8 & 13.7 & 6.3 & 127.3 & 111.2 \\
\hline MPG & 6 & $1.68 \mathrm{E}+07$ & $4.70 \mathrm{E}+05$ & $1.08 \mathrm{E}+08$ & $1.80 \mathrm{E}+06$ & 852 & 45.4 & 24.7 & 17.1 & 5.6 & 147.9 & 130.3 \\
\hline MPG+IMP & 7 & $2.48 \mathrm{E}+07$ & $6.52 \mathrm{E}+05$ & $1.05 \mathrm{E}+08$ & $1.62 \mathrm{E}+06$ & 597 & 53.6 & 24.5 & 9.3 & 5.9 & 176.4 & 125.5 \\
\hline
\end{tabular}

Notes: These data are from the Neurolucida system from each of the 3-D reconstructions of $c$-fos positive nuclei produced throughout the entire taste portion of the PBN after stimulation with 6 different stimuli. This demonstrates that there is extensive variation between the tissue (volume and surface area), distribution, and number of $c$-fos positive nuclei in each brain. One noticeable difference is that DI water stimulation seems to produce less clustered $c-f o s$ positive nuclei in both the SCP and the area of the total PBN subnuclei. 
48 total comparisons. In the $\mathrm{H} 2 \mathrm{O}$ group, the left and right $\mathrm{M}$ subnuclei were significantly different from each other $(\mathrm{t}=-3.849, \mathrm{df}=3, \mathrm{p}=0.031)$. In the $\mathrm{MPG}+10 \mu \mathrm{M}$ amil group, the left and right waist areas were significantly different $(\mathrm{t}=3.914, \mathrm{df}=5, \mathrm{p}=0.011)$ as well as the left and right DL subnuclei $(\mathrm{t}=-2.756, \mathrm{df}=5, \mathrm{p}=0.040)$. In the $\mathrm{MPG}+\mathrm{IMP}+10 \mu \mathrm{M}$ amil group, the left and right EM subnuclei were significantly different from each other $(\mathrm{t}=3.155, \mathrm{df}=5, \mathrm{p}=0.025)$. Finally, in the $\mathrm{QHCl}$ group, the left and right DM subnuclei were significantly different from each other $(Z=2.197, p=0.031)$. The two sides (left and right) of the LC and Me5 were also compared to each other using paired t-tests or Wilcoxon Signed Rank Tests and only one of these comparisons was significantly different. In the MPG $+10 \mu \mathrm{M}$ amil group, the left and right LC were significantly different from each other $(t=-3.316, d f=4, p=0.029)$.

A one-way ANOVA was performed to see if there was a difference between the total number of $c$-fos positive nuclei counted in the PBN response to each of the taste stimuli. It revealed that there was no significant difference between the number of $c$-fos positive nuclei elicited by the six stimuli $(\mathrm{F}(5,29)=0.256, \mathrm{p}=0.934)$ (Figure 4-10A). As controls, Kruskal-Wallis tests were performed on the total number of $c$-fos positive nuclei counted in the LC and the Me5. Neither the LC (Figure 4-10B) $(\mathrm{H}=4.958, \mathrm{df}=5$, $\mathrm{p}=0.421)$ nor the Me5 (Figure 4-10C) $(\mathrm{H}=2.579, \mathrm{df}=5, \mathrm{p}=0.764)$ were found to be different between the six tastant groups. Next, either one-way ANOVAs or KruskalWallis tests followed by Bonferroni or Dunn's post-hoc tests, respectively, were then performed to see if there was a difference between the number of $c$-fos positive nuclei counted in each of the PBN subnuclei in response to each of the taste stimuli. The only subnucleus that a significant difference was found in was the DL subnucleus (Figure 4-11). The number of $c$-fos positive nuclei observed in the DL of the quinine group was statistically significantly lower than in the water group $(\mathrm{df}=5, \mathrm{H}=13.548, \mathrm{p}=0.019)$ (Figure 4-12). Because only one subnucleus was significant in the entire taste portion of the PBN, I wanted to see if other subnuclei were indeed significant in a portion of the PBN, so the data was divided into caudal, intermediate, and rostral levels (Figure 4-13) and reanalyzed using each of the eight subnuclei in these three levels with either one-way ANOVAs or Kruskal-Wallis Tests followed by either Bonferroni or Dunn's post-hoc tests, respectively. There was not a significant difference found in any of the subnuclei in the caudal region of the PBN (Figure 4-13A). In the intermediate level of the PBN, the only significant difference found was in the DL subnucleus, where the number of $c$-fos positive nuclei in the water group was significantly higher than in the QHCl group (Figures 4-13B and 4-14). The rostral level of the PBN is where the most significant differences are found (Figures 4-13C and 4-15). The number of $c$-fos positive nuclei in the waist region of the PBN was significantly higher in the saccharin-stimulated group than it was in both the water- and sucrose-stimulated groups $(\mathrm{df}=5, \mathrm{H}=14.957, \mathrm{p}=0.011)$ (Figure 4-15A). Also, the number of $c$-fos positive nuclei in the CL subnucleus between the stimuli was significantly different, but the Dunn's post-hoc test was not able to ascertain exactly which groups were different $(\mathrm{df}=5, \mathrm{H}=11.608, \mathrm{p}=0.041)$ (Figure 4-15B). Lastly, the number of $c$-fos positive nuclei in the DL subnucleus was significantly higher in the water-stimulated group compared to the QHCl-stimulated group $(\mathrm{F}(5,29)=2.613, \mathrm{p}=0.045)($ Figure 4-15C) . 
A

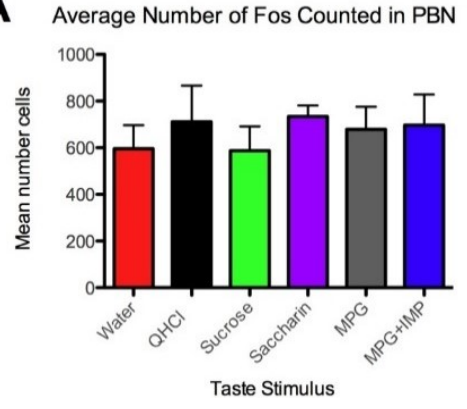

B

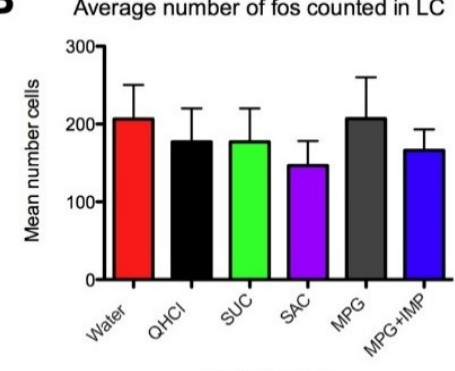

Taste Stimulus
C Average number of fos counted in Me5

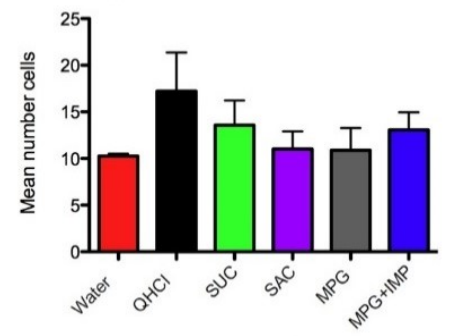

Taste Stimulus

Figure 4-10. Average Number of $C$-fos Counted in the Entire Taste Portion of the PBN, LC, and Me5 for each Taste Stimulus.

Notes: Results of the average total number of $c$-fos positive nuclei counted following taste reactivity stimulation with each of the six different taste stimuli without regards to staining intensity. There was not a significant difference between any of the taste stimuli in the (A) PBN, (B) LC, and (C) Me5. 


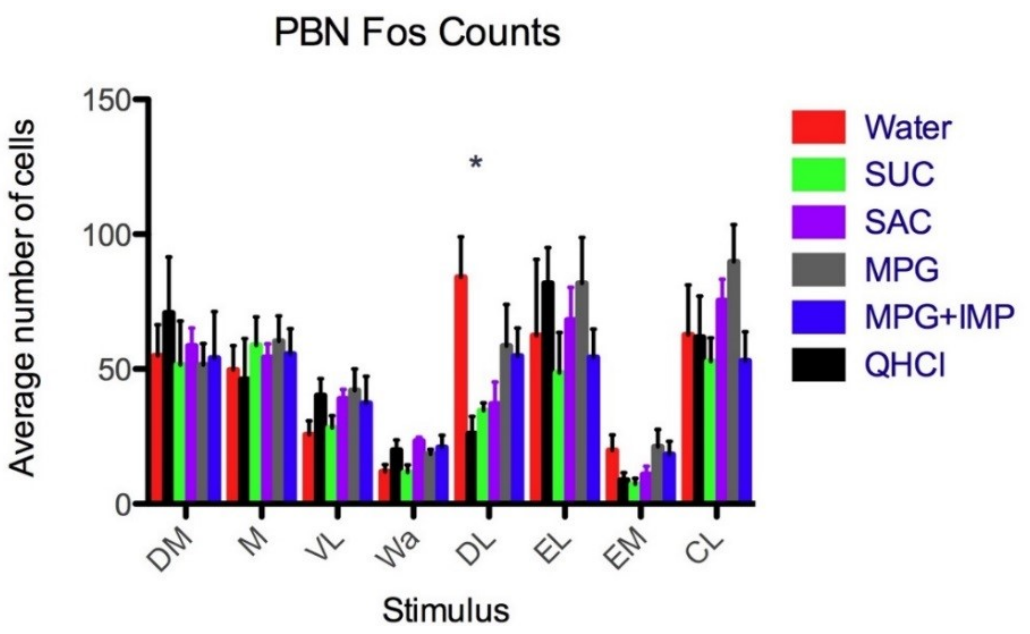

Figure 4-11. Average Number of $C$-fos Counted in Each of the Eight PBN Subnuclei for Each Taste Stimulus.

Notes: The average total number of $c$-fos positive nuclei counted following taste reactivity stimulation with each of the six different taste stimuli without regards to staining intensity. The only subnucleus where a significant difference was found was the DL. $* \mathrm{P}<0.05$. 


\section{Fos Counts DL}

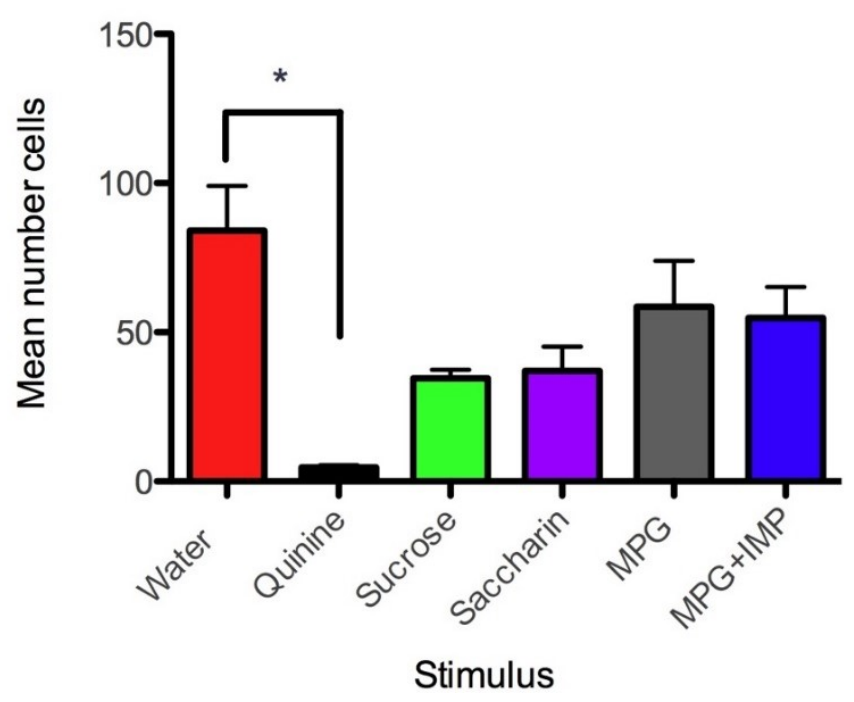

Figure 4-12. Average Number of $C$-fos Counted in DL Subnucleus in the PBN for Each Taste Stimulus.

Notes: The average total number of $c$-fos positive nuclei counted following taste reactivity stimulation with each of the six different taste stimuli in the DL subnucleus. Quinine elicited significantly less $c$-fos positive nuclei than water in the DL subnucleus. $* \mathrm{P}<0.05$. 

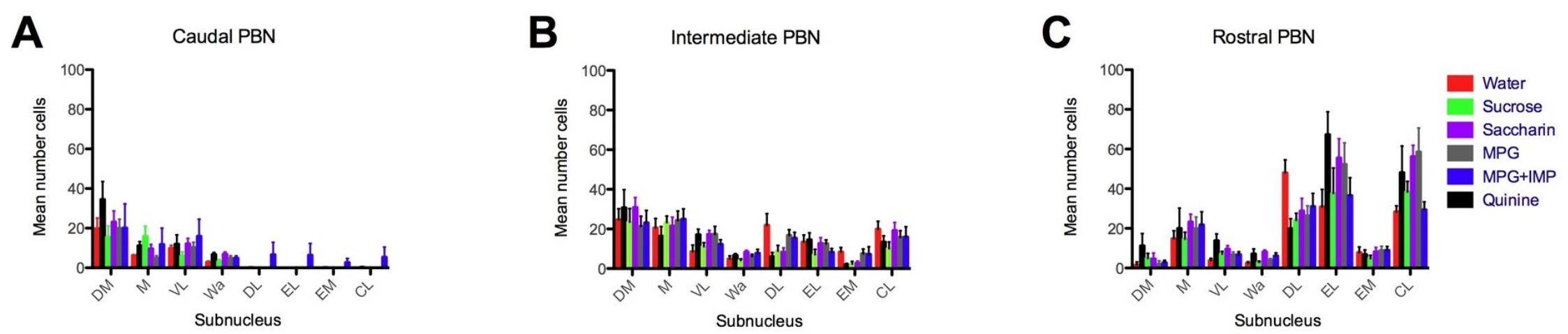

Figure 4-13. The Amount of $C$-fos Expression in the Caudal, Intermediate, and Rostral Levels of Each of the Eight PBN Subnuclei.

Notes: (A) None of the taste stimuli elicited a significantly different number of $c$-fos positive nuclei in any of the eight subnuclei in the caudal portion of the PBN. This graph does show trends emerging, such as quinine eliciting more $c$-fos positive nuclei in the DM subnucleus compared to the rest of the stimuli and sucrose eliciting more $c$-fos positive nuclei in the medial subnucleus compared to the rest of the stimuli in the caudal portion of the PBN. (B) Water elicits significantly more $c$-fos positive nuclei in the DL subnucleus than quinine in the intermediate portion of the PBN. This graph also shows a trend emerging in which quinine elicits more $c-f o s$ positive nuclei in the DM compared to the other taste stimuli. (C) This graph shows trends emerging in which quinine elicits more cfos positive nuclei in the VL and EL compared to water and quinine, and both sucrose and quinine eliciting more $c$-fos positive nuclei in the EL and CL subnucleus compared to water in the rostral portion of the PBN. 


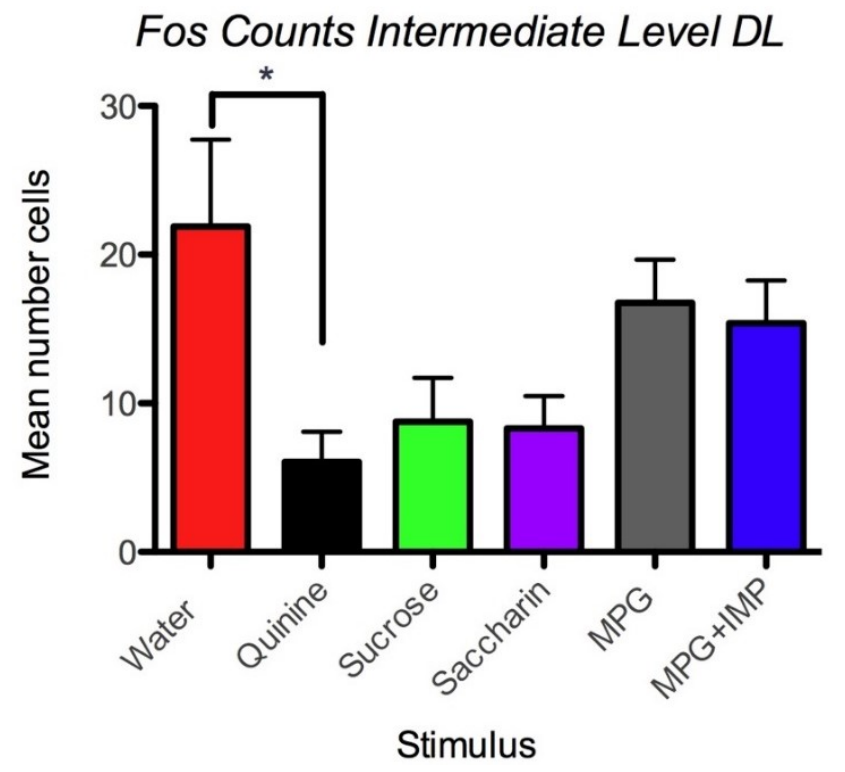

Figure 4-14. The Amount of $C$-fos Expression in the Intermediate Level DL Subnucleus in the PBN.

Notes: Water elicited significantly more $c-f o s$ positive nuclei than quinine did in the DL subnucleus in the $\mathrm{PBN}$. $* \mathrm{P}<0.05$. 
A

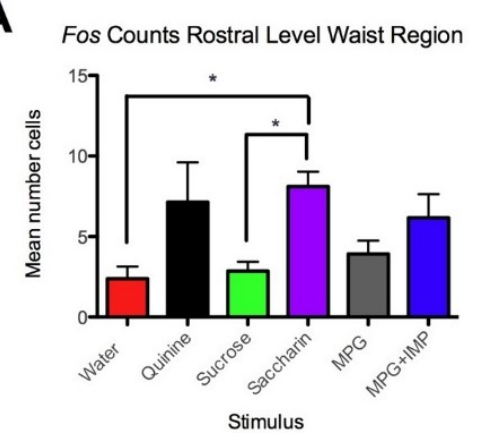

B

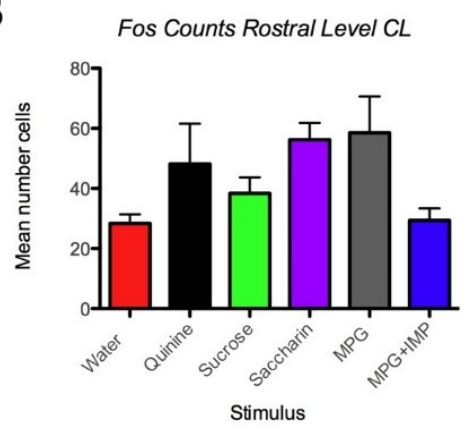

C

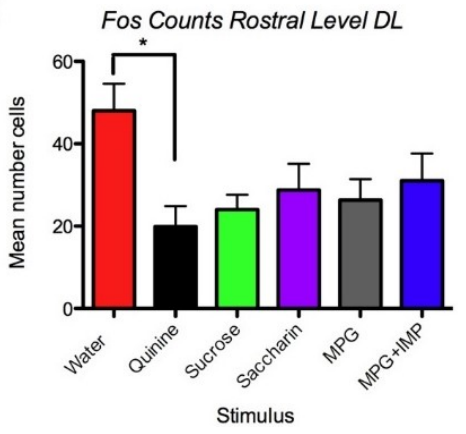

Figure 4-15. The Amount of $C$-fos Expression in the Rostral Level (A) Waist Area and (B) CL subnucleus and the (C) DL Subnucleus in the PBN.

Notes: (A) Saccharin elicited significantly more $c$-fos positive nuclei than both water and sucrose did in the waist region of the PBN. (B) The six taste stimuli elicited significantly different amounts of $c$-fos in the CL subnucleus of the PBN. (C) Quinine produced significantly less $c$-fos positive nuclei than quinine did in the DL subnucleus of the PBN. $* \mathrm{P}<0.05$. 


\section{Discussion}

When investigating the neuroanatomical organization of a particular brain area, certain tools can be utilized to discover which neurons are being activated or affected by a particular stimulus. In the taste field, one tool that has been used in many studies is the marker $c$-fos to visualize which neurons in the specific brain regions are being modified in response to a particular taste stimulus (Hashimoto et al., 2009; Shimura, Tokita, \& Yamamoto, 2002; Tokita, Armstrong, St John, \& Boughter, 2014; Travers et al., 2007; Yamamoto \& Sawa, 2000; Yamamoto et al., 1993; Yamamoto et al., 2009). In the studies presented here, $c$-fos immunochemistry was utilized to visualize these taste-related activation patterns.

In order to investigate the response of neurons in the PBN of mice to sweet, umami, and bitter stimuli, we stimulated mice via implanted intra-oral cannulas and connected the end of it to a pump to administer taste stimuli in a manner that produces forceful ingestion. After the behavioral test was completed, the animals were sacrificed and brain tissue was processed immunohistochemically for the marker $c$-fos. Then the tissue was photographed, PBN subnuclei delineated, and then the $c$-fos positive nuclei within the PBN were counted in the response to DI water, sweet, umami, and bitter stimuli. After finding only a small percentage of significant differences between the right and left side of the PBN (10\%), LC (16.7\%), and Me5 (0\%), the number of $c$-fos positive nuclei counted in both sides of each slice for a particular animal were then added together and averaged. A similar method was used in another study where there were no differences found between the two sides of the PBN following intra-oral stimulation (Anseloni, Ren, Dubner, \& Ennis, 2005). The small amount of differences between the two sides of the brain that were found in this study may reflect some variation among mice but was not deemed to be overly concerning due to the low incidence. As a control, c-fos positive nuclei were also counted in the LC and the Me5 regions near the PBN, which are not related to taste, and found that there was no difference between the number of $c$-fos positive nuclei in these two areas between all of the groups.

I hypothesized that the different taste stimuli would elicit different patterns of $c$ fos positive nuclei in the PBN. More specifically, the sucrose and MPG+IMP $+10 \mu \mathrm{M}$ amil synergistic mixture would elicit similar patterns of $c$-fos positive nuclei that would be more robust than the patterns for MPG and saccharin and that quinine would produce an entirely different pattern from the other taste stimuli tested. There were no significant differences found between the number of total $c$-fos positive nuclei elicited by the individual stimuli in the PBN (Figure 4-10), but when the $c$-fos counts were considered within each subnucleus, QHCl elicited significantly less $c$-fos positive nuclei in the DL subnucleus (Figure 4-12). This finding confirms an earlier study performed in the lab of Dr. Boughter (Tokita et al., 2014). The DL subnucleus has been previously suggested to be involved in ingestion in rats (Yamamoto et al., 1994), so the finding of quinineelicited $c$-fos in this region may be due to forced consumption through the intra-oral cannula, even if they immediately spit out a lot of this stimulus during testing. Also, when rats consumed a familiar stimulus, a large increase in the amount of $c$-fos was found in the DL subnucleus, compared to when they had never had the stimulus. This 
may explain why the mice stimulated with DI water showed a trend of having a higher amount of $c$-fos positive nuclei in the DL subnucleus compared to the rest of the taste stimuli, since the mice were stimulated with DI water twice in the two preceding days before the test day (Yamamoto et al., 2009).

Further analysis of the PBN showed that when the $c$-fos counts were separated into three portions: caudal, intermediate, and rostral levels (Figure 4-13) subtler differences between the taste stimuli were observed. There were still no differences found between any of the subnuclei in the caudal region, but differences were found in the intermediate and rostral portions of the PBN. QHCl elicited significantly less $c$-fos positive nuclei at both the intermediate and rostral levels of the DL (Figures 4-13B and C; Figure 4-14; Figure 4-15C). This demonstrated that the decrease in quinine-elicited $c$-fos positive nuclei occurred in the entire DL subnucleus. In the rostral portion of the waist area $(\mathrm{BC})$, there was an increase in the number of $c$-fos positive nuclei observed with all of the taste stimuli compared to water, however, saccharin elicited significantly more $c$-fos positive nuclei than either sucrose or water (Figure 4-15A). At this level, the BC contains both taste and visceral neurons (Karimnamazi, Travers, \& Travers, 2002), so the increase of $c$-fos positive nuclei could be a combination of both taste and visceral induced $c$-fos positive nuclei from consuming the taste stimuli. There was a significant difference in the number of $c$-fos positive nuclei elicited in the CL in response to the different taste stimuli (Figure 4-15B). In contrast to the BC, the CL subnucleus in rats has been indicated as an area in which pain and inflammation can induce an increase in $c$ fos positive nuclei in the CL. (Bellavance \& Beitz, 1996). It is possible that inflammation and/or pain from the intra-oral cannula or from the sensation of fluid moving through the cannula and into the mouth could have influenced the level of $c$-fos positive nuclei in this particular area. In the current study only 5 days elapsed before testing instead of 7 days, as was done in some previous studies in hamsters and rats (Brining et al., 1991; Grill \& Norgren, 1978a). In addition, the introduction of novel taste stimuli could have caused a sympathetic response in the mice, which in turn produced these increases in the $c$-fos expression in the CL subnucleus (Davern, 2014). It has been found previously in rats that sweet stimuli elicit $c$-fos in the CL subnucleus (Bureš, Bermudez-Rattoni, \& Yamamoto, 1998). Although I did replicate the finding of a significantly decreased level of $c$-fos positive nuclei in response to $\mathrm{QHCl}$ in the DL subnucleus, this study did not find as many differences as the previous study performed in our lab in mice and also quantified a greater amount of $c$-fos positive cells in the PBN subnuclei (Tokita et al., 2014). As was observed in rats, I did not observe specific taste maps for each different taste stimulus similar to a previous study by Yamamoto et al., using rats (Yamamoto et al., 1994). This could be due to many factors. First, the number of animals within a few of the groups were probably too small to reveal subtle differences, compounded by the fact that there was good evidence for a high level of animal-to-animal variability in $c$-fos expression in general (Bures, 1998). Secondly, there may be a species difference in $c$-fos expression in the PBN subnuclei between rats and mice. Thirdly, there were differences between the stimulus concentrations and flow rate used. Lastly, the length of time the stimulation occurred (15 minutes vs. 20 minutes) was longer,potentially causing more neurons to be activated. The final difference, and perhaps the greatest was the differences in staining technique, with the DAB allowing for a more defined imaging vs. fluorescent 
immunohistochemistry, for the total number of neurons, subnuclear differentiation, and intensity of the $c$-fos positive nuclei that were counted.

The subnuclei (BC, DL, and CL) where $c$-fos positive nuclei differences were located are areas that have been found to be involved in processing both taste and visceral information (Halsell \& Travers, 1997; Herbert et al., 1990; Karimnamazi et al., 2002). Using an intra-oral cannula connected to a pump to produce a consistent level of taste stimulation forces the mice to consume taste stimuli in possibly larger quantities then they would naturally (especially in the case of aversive stimuli) and not only activates the taste receptors in the mouth but also results in visceral activation (Tokita et al., 2014; Yamamoto \& Sawa, 2000). Yamamoto compared intra-oral and intragastric $c$-fos in rats elicited by sweet and bitter stimuli and found that there was a portion of the $c$-fos that was elicited by taste, but it is just not possible to tell which portions are contributed by each in this experiment (Yamamoto \& Sawa, 2000). Additional $c$-fos activation may be caused by the pain, cardiovascular and respiratory systems (Dawid Milner et al., 2003; Hermanson \& Blomqvist, 1997). The DL and CL subnuclei are innervated by the afferent projections from pain-responding neurons in the spinal dorsal horn (Cechetto, Standaert, \& Saper, 1985; Slugg \& Light, 1994). Formalin injections into the lip of rats have been found to produce $c$-fos in the CL and DL subnuclei (Hermanson \& Blomqvist, 1997). There was a trend of an increase in $c$-fos in the right side of the CL subnucleus in the PBN (where the cannula was placed) compared to the left side in all of the groups except for water, although this difference was not significant. Also, electrical stimulation in the lateral PBN subnuclei produces changes in cardiorespiratory responses in rats (Dawid Milner et al., 2003).

In electrophysiological experiments performed in mice, taste neurons found responsive to only oral stimulation have been found in a few subnuclei, consisting of the caudal waist region and rostral portions of the M, VL, and EL subnuclei (Tokita \& Boughter, 2012; Tokita et al., 2012). An anatomical study also found retrogradely labeled cells in the same subnuclei after injection with wheat germ agglutinin-conjugated horseradish peroxidase into the taste portion of the thalamus (Hashimoto et al., 2009). It is possible that the CL and DL subnuclei do contain some taste neurons, but they have not been thoroughly explored yet electrophysiologically, and the small size of these subnuclei may make it hard to record from them. However, one study in hamsters did not find taste activity in the DL and CL subnuclei (Halsell \& Frank, 1991).

The tissue used to delineate the subnuclei had each individual section counted and was then visually examined by first comparing sections from a similar level in the intermediate PBN in all of the six different taste stimuli, which displayed a similar $c-f o s$ pattern in the sucrose, saccharin, and MPG+IMP-stimulated animals, and a more intense and widespread c-fos pattern in the quinine-stimulated animals (Figure 4-3). Next, the slices were used to produce $3 \mathrm{D}$ reconstructions of an individual animal for each of the six taste stimuli. In order to make sure the brain sections of the PBN were stacked on top of each other properly, a three-dimensional model was created by scanning sections from the Paxinos Brain Atlas containing the portion of the PBN quantified in this study. Creating the three-dimensional images of the PBN gave a better idea of the relation of the 
$c$-fos positive nuclei to each other throughout the subnuclei and has the potential to give the investigator other ways to find differences between stimuli instead of just looking at increases or decreases in $c$-fos positive nuclei numbers. These figures seemed to better illustrate the subtle differences between the taste stimuli, compared to the twodimensional slices shown in Figure 4-3. This method, may, in fact, represent a better way to quantify all of this data. However, the $3 \mathrm{D}$ reconstruction is also time- and laborintensive, relying on a shared facility. Moreover, ways to quantify meaningful variation in parameters like cell spacing or density would need to be developed, as well as a method to merge all of the images so it is easier to compare the different stimuli. Two previous studies have utilized this method in the NST. One used the software Matlab, which requires programming expertise (Kwak et al., 2015), and the other used a French software called Free-D, which was found to not be user-friendly (Schwarz et al., 2010). The study using Matlab created three-dimensional images, but the analysis was still performed on two-dimensional sections, similar to the current study. In the future, the best option would be to obtain training on using the Free-D software and use it to create three-dimensional models of averages of all of the animals stimulated in each group, to account for the inter-animal variability, and analyze density maps of the $c$-fos positive nuclei in the different areas of the PBN. If there is indeed no difference between the stimuli in the three-dimensional images, then it is possible that the $c$-fos protein is not sensitive enough to elucidate differential maps according to taste stimuli in this brain area. Still, the potential for uncovering subtle variation in expression using 3D analysis makes this method attractive for future studies in the gustatory system.

Overall, this study did not find differences in taste specific subnuclei in terms of differential $c$-fos expression produced by water, sweet, umami, and bitter stimuli. There was also no evidence of synergistic effects, similar to the taste reactivity behavior shown in Chapter 3 - in other words, MPG+IMP did not elicit more $c$-fos positive nuclei compared to MPG individually. This non-effect in terms of $c$-fos expression may in part reflect limitations of using the $c$-fos technique itself, such as the fact that transcription of the $c$-fos protein is only indicative of a response in the cell to a stimulus, but not the strength of the stimulus. Nor does it reflect whether the stimulus exposure was long enough to induce $c$-fos since electrophysiology of a neuron does not always parallel $c$-fos production (Fenelon, Poulain, \& Theodosis, 1993). Also, there could be taste responses in cells that do not produce the $c$-fos protein, i.e. parts of the stimulus response that are not being seen when only examining $c$-fos positive nuclei (Kaufman, 2005). Lastly, since the $\mathrm{PBN}$ is involved in so many different functions, some of the $c$-fos positive nuclei found in this study could be a result of some other accidental stimulus besides taste, i.e. ingestion, which could make it hard to see a difference. 


\section{CHAPTER 5. FINAL SUMMARY AND OVERALL CONCLUSIONS}

The experiments described in the previous chapters were focused on determining if there were similarities between how B6 mice behaviorally perceive sweet and umami taste in B6 mice, and whether these stimuli had similar neuroanatomical substrates. The taste reactivity and two-bottle preference tests were designed to investigate the palatability and preference of a taste stimulus by evaluating behaviors (consumption and facial reactions) in response to administration. In the experiments in this dissertation, I used both behavioral and neuroanatomical methods to compare a variety of taste stimuli. The taste of umami has been studied the least out of the five basic taste qualities and the majority of studies that have been performed have used rats as subjects, rather than mice.

Prior electrophysiological studies performed in our lab found that the response of isolated gustatory neurons in the PBN to MPG+IMP was very similar to the response to sucrose (Tokita \& Boughter, 2012; Tokita et al., 2012). This finding, combined with other evidence stated previously in Chapter 1, seemed to indicate that sweet and synergistic umami mixtures are perceived as similar in mice, even though they exhibit completely different taste qualities to humans. I hypothesized, therefore, that sweet and umami mixture stimuli would produce similar $c$-fos expression patterns in B6 mice, and that these patterns would also be different from both bitter stimuli and water.

As a previous study from this laboratory assessed taste response to these stimuli using a CTA licking task, I sought to extend the understanding of the behavioral dimensions of sweet and umami stimuli by measuring preference and consumption- this approach should yield hedonic parameters such as avidity and aversion. I found that B6 mice prefer (to water) all of the umami and sweet compounds that were tested, but preferred and consumed the synergistic mixture of MSG+IMP the most out of all of the umami stimuli, and preferred and consumed this umami compound to almost the same level as they preferred the higher concentration of sucrose. This finding agreed with a prior study on preference for sweet stimuli, MSG, and IMP (Bachmanov, Tordoff, \& Beauchamp, 2001). I also replicated earlier work showing that B6 mice strongly avoid (and do not consume) quinine (Tordoff, 2007). Moreover, these results also revealed a species difference between mice and rats in regards to preference for umami stimuli. Mice seem to prefer MSG and MPG more and at higher concentrations than do rats (Grill \& Flynn, 1987; Miura et al., 2014).

A second behavioral test (taste reactivity) was used to gain insights into the apparent behavioral similarity between sweet and synergistic umami stimuli. Here, however, after examining overall taste reactivity using sweet, umami, and bitter stimuli, along with water, I found that only quinine produced a distinct variety of behaviors among the set of the stimuli. Even though the sweet and umami stimuli appeared to be of similar palatability as indicated by the taste reactivity behaviors elicited, they were not significantly different from water. Interestingly, analyzing the data in a time-dependent manner did reveal some noticeable differences between the sweet and umami taste reactivity elicited behaviors, and between the bitter elicited taste reactivity behaviors and 
the rest of the taste stimuli. These results indicate that measuring taste reactivity in mice shows a clear aversive phenotype for bitter stimuli; this is a useful consideration for neuroanatomical studies, which rely on forced, intra-oral simulation, as voluntary consumption would produce differential amounts of taste receptor stimulation, which would produce a confound in terms of measuring an activity-dependent marker like $c$-fos.

There were several important limitations to the taste reactivity behavioral approach. One was that the size and natural behaviors of the mice seemed to preclude observation of some of the taste reactivity behaviors (lateral tongue protrusions, tongue protrusions, gaping, and paw pushing) that have been quantified in rats previously. These behaviors may be very important in seeing differences or similarities between taste stimuli. Secondly, the dark pigment in B6 mice also made it difficult to see mouth movements. It is possible that these limitations could be overcome with video acquisition that has higher resolution than I used, or with electromyography from orofacial musculature. Other behavioral tests such as an operant conditioning-based stimulus discrimination approach could also provide insight into how mice perceive umami stimuli, especially as it is currently suspected that their perception of this taste quality is not as distinct as in humans.

The second focus of this study was to examine $c$-fos expression in the PBN, an important relay in the taste pathway, following intra-oral cannula stimulation with sweet, bitter, and umami stimuli along with water. I also wanted to use some of the tissue to create three-dimensional c-fos maps in the PBN, to see if this type of reconstruction or visualization of cellular expression data would allow for additional insight. I quantified $c$ fos positive nuclei in in all eight of the subnuclei in the PBN in 5-7 sections containing rostral, intermediate, and caudal portions of the PBN: DM, M, VL, DL, waist, EM, EL, and CL. In summary, I only found differences in the entire DL subnucleus, the intermediate level of the DL subnucleus, and the rostral levels of the waist, DL, and CL subnuclei. The decrease seen in c-fos positive cells in the DL subnucleus after QHCl stimulation relative to other stimuli was also seen previously in a separate study performed in our lab (Tokita et al., 2014). One interesting finding was an increase in $c$-fos positive cells in the DL subnucleus in the water group. This may be due to mice considering water as a familiar stimulus since higher levels of $c$-fos were found previously in rats after consuming a familiar stimulus and not when consuming a novel one (Yamamoto et al., 2009). In comparison to the 2D level-specific counts, the threedimensional $c$-fos maps I created may afford a better representation of the overall $c$-fos pattern, leading to insights into subtle variation among stimuli.

There were several limitations to the $c$-fos study. The first is the $c$-fos technique itself. There was variability between $c$-fos levels in animals within the same stimulus group. This variability may occlude finding significant differences in expression between taste qualities. Also, even though this technique is a high-throughput way of characterizing cells throughout the entire brain that responded to a stimulus at a particular point in time, finding $c$-fos positive nuclei is only an indication of whether a cell was activated or not, but does not yield information about the strength of the response, or if there were activated cells that do not produce the $c$-fos protein in response to stimuli. It is 
possible that in this manner critical information may be missed. Also, it is possible that the timing of collection of the $c$-fos data ( 2 hours after taste stimulation) may have produced more visceral-evoked $c$-fos in this study. An experiment varying the length of perfusion time from 1 hour to 2 hours after testing might reveal if the timing is indeed an issue in terms of the number/location of $c$-fos positive nuclei. Finally, the behavioral technique used in this study to produce taste stimulation, taste reactivity, may also be a limitation. This technique requires a surgery to implant the intra-oral cannula into the mouth, which is then used to introduce taste stimuli into the oral cavity. It is possible that this method of taste stimulation although necessary for adequate stimulation especially with aversive compounds, may induce non-gustatory $c$-fos expression, such as that produced by pain from the surgical site, stress from forced consumption, or post-ingestive factors from the same. Of the three portions of the PBN that we saw differences in, two subnuclei (DL and CL) have not been implicated in taste, but instead in visceral processes in addition to taste (Dawid Milner et al., 2003; Hermanson \& Blomqvist, 1997; Yamamoto \& Sawa, 2000). Future studies could be performed to parse out some of these other functions of the PBN, including expression that is non-gustatory in nature. Other behavioral techniques could be included such as lick assays for preferred stimuli, or stomach gavage for estimating visceral-only stimulation. In addition, labeling for both $c$ fos and another immediate early gene, such as Egr-1 or arg 3.1, and labeling mRNA instead of the $c$-fos protein may help to get a better picture of the events occurring in response to taste stimuli.

In conclusion, it was first hypothesized that sweet and umami stimuli (especially the synergistic mixtures) would be preferred at a similar level and elicit similar levels and kinds of taste reactivity behaviors. The results from these two behavioral experiments showed this hypothesis to be partially correct. The first experiment showed that B6 mice do highly prefer the sweet (sucrose and saccharin) and umami stimuli (MSG, MPG, and synergistic mixtures of both with IMP). The most striking example of this is that both 0.3 M MSG+amil and the synergistic mixture of $0.3 \mathrm{M} \mathrm{MSG+IMP+amil} \mathrm{are} \mathrm{both} \mathrm{consumed}$ at the same high level as $0.1 \mathrm{M}$ sucrose. The second experiment, however, did not support the hypothesis since the results indicated that the major difference found in taste reactivity behavior was between five of the taste stimuli (sucrose, saccharin, MPG+amil, MPG+IMP+amil, and water) and the bitter stimulus, QHCl. The second hypothesis was that sweet and umami stimuli would produce similar patterns of $c$-fos activation in the PBN of B6 mice, and the other stimuli would produce different patterns of $c$-fos activation. Overall, there was only one difference between all of the PBN subnuclei and that was in response to $\mathrm{QHCl}$. When the subnuclei were divided into three different portions there were only three rostral subnuclei in the PBN that differences were found in. All of these areas have been previously found to be involved in both taste and visceral processes, so it is difficult to tell exactly what produced these differences. Therefore, these results did not support the hypothesis. 


\section{LIST OF REFERENCES}

Accolla, R., Bathellier, B., Petersen, C. C., \& Carleton, A. (2007). Differential spatial representation of taste modalities in the rat gustatory cortex. J Neurosci, 27(6), 1396-1404. doi:10.1523/JNEUROSCI.5188-06.2007

Adler, E., Hoon, M. A., Mueller, K. L., Chandrashekar, J., Ryba, N. J., \& Zuker, C. S. (2000). A novel family of mammalian taste receptors. Cell, 100(6), 693-702.

Anseloni, V. C., Ren, K., Dubner, R., \& Ennis, M. (2005). A brainstem substrate for analgesia elicited by intraoral sucrose. Neuroscience, 133(1), 231-243. doi:10.1016/j.neuroscience.2005.01.055

Armario, A. (2006). The Contribution of Immediate Early Genes to the Understanding of Brain Processing of Stressor. In R. Pinaud \& L. A. Tremere (Eds.), Immediate Early Genes in Sensory Processing, Cognitive Performance and Neurological Disorders (pp. 199-221). New York, NY: Springer Business Science Media, LLC.

Bachmanov, A. (2010). Umami: Fifth Taste?

Flavor Enhancer? Perfumer \& Flavorist, 35.

Bachmanov, A. A., Li, X., Reed, D. R., Ohmen, J. D., Li, S., Chen, Z., . . Beauchamp, G. K. (2001). Positional cloning of the mouse saccharin preference (Sac) locus. Chem Senses, 26(7), 925-933.

Bachmanov, A. A., Reed, D. R., Beauchamp, G. K., \& Tordoff, M. G. (2002). Food intake, water intake, and drinking spout side preference of 28 mouse strains. Behav Genet, 32(6), 435-443.

Bachmanov, A. A., Reed, D. R., Ninomiya, Y., Inoue, M., Tordoff, M. G., Price, R. A., \& Beauchamp, G. K. (1997). Sucrose consumption in mice: major influence of two genetic loci affecting peripheral sensory responses. Mamm Genome, 8(8), 545548.

Bachmanov, A. A., Tordoff, M. G., \& Beauchamp, G. K. (2000). Intake of umami-tasting solutions by mice: a genetic analysis. $J$ Nutr, 130(4S Suppl), 935S-941S.

Bachmanov, A. A., Tordoff, M. G., \& Beauchamp, G. K. (2001). Sweetener preference of C57BL/6ByJ and 129P3/J mice. Chem Senses, 26(7), 905-913.

Baird, J. P., Travers, S. P., \& Travers, J. B. (2001). Integration of gastric distension and gustatory responses in the parabrachial nucleus. Am J Physiol Regul Integr Comp Physiol, 281(5), R1581-1593. 
Beauchamp, G. K., \& Mennella, J. A. (2011). Flavor perception in human infants: development and functional significance. Digestion, 83 Suppl 1, 1-6. doi:10.1159/000323397

Beckstead, R. M., Morse, J. R., \& Norgren, R. (1980). The nucleus of the solitary tract in the monkey: projections to the thalamus and brain stem nuclei. J Comp Neurol, 190(2), 259-282. doi:10.1002/cne.901900205

Bellavance, L. L., \& Beitz, A. J. (1996). Altered c-fos expression in the parabrachial nucleus in a rodent model of CFA-induced peripheral inflammation. J Comp Neurol, 366(3), 431-447. doi:10.1002/(SICI)10969861(19960311)366:3\&lt;431::AID-CNE5\&gt;3.0.CO;2-5

Berridge, K., Grill, H. J., \& Norgren, R. (1981). Relation of consummatory responses and preabsorptive insulin release to palatability and learned taste aversions. J Comp Physiol Psychol, 95(3), 363-382.

Berridge, K. C. (1991). Modulation of taste affect by hunger, caloric satiety, and sensoryspecific satiety in the rat. Appetite, 16(2), 103-120.

Berridge, K. C. (2000). Measuring hedonic impact in animals and infants: microstructure of affective taste reactivity patterns. Neurosci Biobehav Rev, 24(2), 173-198.

Berridge, K. C., Flynn, F. W., Schulkin, J., \& Grill, H. J. (1984). Sodium depletion enhances salt palatability in rats. Behav Neurosci, 98(4), 652-660.

Berridge, K. C., \& Treit, D. (1986). Chlordiazepoxide directly enhances positive ingestive reactions in rats. Pharmacol Biochem Behav, 24(2), 217-221.

Birch, G. C. (1987). Structure, Chirality, and Solution Properties of Glutamates in Relation to Taste. In Y. Kawamura \& M. R. Kare (Eds.), Umami: A Basic Taste: Physiology, Biochemistry, Nutrition, Food Science (pp. 173-183). New York, NY: Marcel Dekker.

Blizard, D. A., Kotlus, B., \& Frank, M. E. (1999). Quantitative trait loci associated with short-term intake of sucrose, saccharin and quinine solutions in laboratory mice. Chem Senses, 24(4), 373-385.

Breslin, P. A., Spector, A. C., \& Grill, H. J. (1992). A quantitative comparison of taste reactivity behaviors to sucrose before and after lithium chloride pairings: a unidimensional account of palatability. Behav Neurosci, 106(5), 820-836.

Breza, J. M., Curtis, K. S., \& Contreras, R. J. (2007). Monosodium glutamate but not linoleic acid differentially activates gustatory neurons in the rat geniculate ganglion. Chem Senses, 32(9), 833-846. doi:10.1093/chemse/bjm052 
Brining, S. K., Belecky, T. L., \& Smith, D. V. (1991). Taste reactivity in the hamster. Physiol Behav, 49(6), 1265-1272.

Bullitt, E. (1990). Expression of $c$-fos-like protein as a marker for neuronal activity following noxious stimulation in the rat. J Comp Neurol, 296(4), 517-530. doi:10.1002/cne.902960402

Bureš, J., Bermudez-Rattoni, F., \& Yamamoto, T. (1998). Functional Morphology of CTA. In Conditioned taste aversion: Memory of a special kind (pp. 92-107). Oxford: Oxford University Press.

Cabanac, M., \& Lafrance, L. (1990). Postingestive alliesthesia: the rat tells the same story. Physiol Behav, 47(3), 539-543.

Cabanac, M., \& Lafrance, L. (1991). Facial consummatory responses in rats support the ponderostat hypothesis. Physiol Behav, 50(1), 179-183.

Cairncross, S. E., \& Sjostrom, L. B. (1948). What glutamate does in food. Food Ind, $20(7), 982$.

Capeless, C. G., \& Whitney, G. (1995). The genetic basis of preference for sweet substances among inbred strains of mice: preference ratio phenotypes and the alleles of the Sac and dpa loci. Chem Senses, 20(3), 291-298.

Caston-Balderrama, A. L., Cameron, J. L., \& Hoffman, G. E. (1998). Immunocytochemical localization of Fos in perfused nonhuman primate brain tissue: fixation and antisera selection. J Histochem Cytochem, 46(4), 547-556.

Cechetto, D. F., Standaert, D. G., \& Saper, C. B. (1985). Spinal and trigeminal dorsal horn projections to the parabrachial nucleus in the rat. J Comp Neurol, 240(2), 153-160. doi:10.1002/cne.902400205

Chan, C. Y., Yoo, J. E., \& Travers, S. P. (2004). Diverse bitter stimuli elicit highly similar patterns of Fos-like immunoreactivity in the nucleus of the solitary tract. Chem Senses, 29(7), 573-581. doi:10.1093/chemse/bjh062

Chandrashekar, J., Mueller, K. L., Hoon, M. A., Adler, E., Feng, L., Guo, W., . . Ryba, N. J. (2000). T2Rs function as bitter taste receptors. Cell, 100(6), 703-711.

Chaudhari, N., Landin, A. M., \& Roper, S. D. (2000). A metabotropic glutamate receptor variant functions as a taste receptor. Nat Neurosci, 3(2), 113-119.

doi:10.1038/72053

Chaudhari, N., Yang, H., Lamp, C., Delay, E., Cartford, C., Than, T., \& Roper, S. (1996). The taste of monosodium glutamate: membrane receptors in taste buds. $J$ Neurosci, 16(12), 3817-3826. 
Cromwell, H. C., \& Berridge, K. C. (1993). Where does damage lead to enhanced food aversion: the ventral pallidum/substantia innominata or lateral hypothalamus? Brain Res, 624(1-2), 1-10.

Curran, T., MacConnell, W. P., van Straaten, F., \& Verma, I. M. (1983). Structure of the FBJ murine osteosarcoma virus genome: molecular cloning of its associated helper virus and the cellular homolog of the v-fos gene from mouse and human cells. Mol Cell Biol, 3(5), 914-921.

Damak, S., Rong, M., Yasumatsu, K., Kokrashvili, Z., Perez, C. A., Shigemura, N., . . . Margolskee, R. F. (2006). Trpm5 null mice respond to bitter, sweet, and umami compounds. Chem Senses, 31(3), 253-264. doi:10.1093/chemse/bjj027

Damak, S., Rong, M., Yasumatsu, K., Kokrashvili, Z., Varadarajan, V., Zou, S., . . Margolskee, R. F. (2003). Detection of sweet and umami taste in the absence of taste receptor T1r3. Science, 301(5634), 850-853. doi:10.1126/science.1087155

Dampney, R. A. (1994). Functional organization of central pathways regulating the cardiovascular system. Physiol Rev, 74(2), 323-364.

Danilova, V., Damak, S., Margolskee, R. F., \& Hellekant, G. (2006). Taste responses to sweet stimuli in alpha-gustducin knockout and wild-type mice. Chem Senses, 31(6), 573-580. doi:10.1093/chemse/bjj062

Davern, P. J. (2014). A role for the lateral parabrachial nucleus in cardiovascular function and fluid homeostasis. Front Physiol, 5, 436. doi:10.3389/fphys.2014.00436

Dawid Milner, M. S., Lara, J. P., Lopez de Miguel, M. P., Lopez-Gonzalez, M. V., Spyer, K. M., \& Gonzalez-Baron, S. (2003). A5 region modulation of the cardiorespiratory responses evoked from parabrachial cell bodies in the anaesthetised rat. Brain Res, 982(1), 108-118.

de Araujo, I. E. (2009). Gustatory and homeostatic functions of the rodent parabrachial nucleus. Ann N Y Acad Sci, 1170, 383-391. doi:10.1111/j.1749-

6632.2009.03923.x

Delay, E. R., Hernandez, N. P., Bromley, K., \& Margolskee, R. F. (2006). Sucrose and monosodium glutamate taste thresholds and discrimination ability of T1R3 knockout mice. Chem Senses, 31(4), 351-357. doi:10.1093/chemse/bjj039

Delwiche, J. F. (2012). You eat with your eyes first. Physiol Behav, 107(4), 502-504. doi:10.1016/j.physbeh.2012.07.007 
Di Lorenzo, P. M., Platt, D., \& Victor, J. D. (2009). Information processing in the parabrachial nucleus of the pons. Ann N Y Acad Sci, 1170, 365-371.

doi:10.1111/j.1749-6632.2009.03903.x

Doyle, T. G., Berridge, K. C., \& Gosnell, B. A. (1993). Morphine enhances hedonic taste palatability in rats. Pharmacol Biochem Behav, 46(3), 745-749.

Fenelon, V. S., Poulain, D. A., \& Theodosis, D. T. (1993). Oxytocin neuron activation and Fos expression: a quantitative immunocytochemical analysis of the effect of lactation, parturition, osmotic and cardiovascular stimulation. Neuroscience, 53(1), 77-89.

Fernstrom, J. D., Munger, S. D., Sclafani, A., de Araujo, I. E., Roberts, A., \& Molinary, S. (2012). Mechanisms for sweetness. $J$ Nutr, 142(6), 1134S-1141S.

doi:10.3945/jn.111.149567

Fields, R. D., Eshete, F., Dudek, S., Ozsrac, N., \& Stevens, B. (2001). Regulation of gene expression of action potentials: Dependence of complexity in action potential firing. In G. Bock \& J. Goode (Eds.), Complexity in Biological Information Processing (pp. 160-172). Chichester, England: John Wiley \& Sons.

Fuller, J. L. (1974). Single-locus control of saccharin preference in mice. J Hered, 65(1), 33-36.

Fulwiler, C. E., \& Saper, C. B. (1984). Subnuclear organization of the efferent connections of the parabrachial nucleus in the rat. Brain Res, 319(3), 229-259.

Ganchrow, J. R., Steiner, J. E., \& Bartana, A. (1990). Behavioral reactions to gustatory stimuli in young chicks (Gallus gallus domesticus). Dev Psychobiol, 23(2), 103 117. doi:10.1002/dev.420230202

Ganchrow, J. R., Steiner, J. E., \& Canetto, S. (1986). Behavioral displays to gustatory stimuli in newborn rat pups. Dev Psychobiol, 19(3), 163-174.

doi:10.1002/dev.420190303

Geran, L. C., \& Travers, S. P. (2009). Bitter-responsive gustatory neurons in the rat parabrachial nucleus. J Neurophysiol, 101(3), 1598-1612.

doi:10.1152/jn.91168.2008

Greenberg, M. E., \& Ziff, E. B. (1984). Stimulation of 3 T3 cells induces transcription of the $c$-fos proto-oncogene. Nature, 311(5985), 433-438.

Grigson, P. S., Reilly, S., Shimura, T., \& Norgren, R. (1998). Ibotenic acid lesions of the parabrachial nucleus and conditioned taste aversion: further evidence for an associative deficit in rats. Behav Neurosci, 112(1), 160-171. 
Grill, H. J. (1985). Introduction: physiological mechanisms in conditioned taste aversions. Ann N Y Acad Sci, 443, 67-88.

Grill, H. J., \& Norgren, R. (1978a). The taste reactivity test. I. Mimetic responses to gustatory stimuli in neurologically normal rats. Brain Res, 143(2), 263-279.

Grill, H. J., \& Norgren, R. (1978b). The taste reactivity test. II. Mimetic responses to gustatory stimuli in chronic thalamic and chronic decerebrate rats. Brain Res, 143(2), 281-297.

Grill, H. J., \& Berridge, K. C. (1985). Taste Reactivity as a Measure of the Neural Control of Palatability. In J. M. Sprague \& A. N. Epstein (Eds.), Progress in Psychobiology and Physiological Psychology (Vol. 11, pp. 1-61). Orlando, FL: Academic Press.

Grill, H. J., \& Flynn, F. W. (1987). Behavioral Analysis of Oral Stimulating Effects of Amino Acid and Glutamate Compounds on the Rat. In Y. Kawamura \& M. R. Kare (Eds.), Umami: A Basic Taste: Physiology, Biochemistry, Nutrition, Food Science (pp. 461-480). New York, NY: Marcel Dekker.

Grill, H. J., Flynn, F. W., \& Schwartz, G. J. (1987). Taste effects of some amino acids and glutamate compounds in the rat. Chem Senses Chemical Senses, 12(2), 307322. doi:10.1093/chemse/12.2.307

Grobe, C. L., \& Spector, A. C. (2008). Constructing quality profiles for taste compounds in rats: a novel paradigm. Physiol Behav, 95(3), 413-424.

doi:10.1016/j.physbeh.2008.07.007

Haino, T., Hironaka, S., Ooka, T., Tokita, K., Kubota, Y., Boughter, J. D., Jr., . . Mukai, Y. (2010). Orosensory deprivation alters taste-elicited $c$-Fos expression in the parabrachial nucleus of neonatal rats. Neurosci Res, 67(3), 228-235. doi:10.1016/j.neures.2010.03.007

Hajnal, A., \& Norgren, R. (2005). Taste pathways that mediate accumbens dopamine release by sapid sucrose. Physiol Behav, 84(3), 363-369. doi:10.1016/j.physbeh.2004.12.014

Hajnal, A., Takenouchi, K., \& Norgren, R. (1999). Effect of intraduodenal lipid on parabrachial gustatory coding in awake rats. J Neurosci, 19(16), 7182-7190.

Halsell, C. B. (1992). Organization of parabrachial nucleus efferents to the thalamus and amygdala in the golden hamster. J Comp Neurol, 317(1), 57-78.

doi:10.1002/cne.903170105 
Halsell, C. B., \& Frank, M. E. (1991). Mapping study of the parabrachial taste-responsive area for the anterior tongue in the golden hamster. J Comp Neurol, 306(4), 708722. doi:10.1002/cne.903060412

Halsell, C. B., \& Travers, S. P. (1997). Anterior and posterior oral cavity responsive neurons are differentially distributed among parabrachial subnuclei in rat. $J$ Neurophysiol, 78(2), 920-938.

Halsell, C. B., Travers, S. P., \& Travers, J. B. (1996). Ascending and descending projections from the rostral nucleus of the solitary tract originate from separate neuronal populations. Neuroscience, 72(1), 185-197.

Hamilton, R. B., \& Norgren, R. (1984). Central projections of gustatory nerves in the rat. J Comp Neurol, 222(4), 560-577. doi:10.1002/cne.902220408

Hashimoto, K., Obata, K., \& Ogawa, H. (2009). Characterization of parabrachial subnuclei in mice with regard to salt tastants: possible independence of taste relay from visceral processing. Chem Senses, 34(3), 253-267. doi:10.1093/chemse/bjn085

Hayward, L. F., \& Castellanos, M. (2003). Increased c-Fos expression in select lateral parabrachial subnuclei following chemical versus electrical stimulation of the dorsal periaqueductal gray in rats. Brain Res, 974(1-2), 153-166.

He, W., Yasumatsu, K., Varadarajan, V., Yamada, A., Lem, J., Ninomiya, Y., . . Damak, S. (2004). Umami taste responses are mediated by alpha-transducin and alpha-gustducin. J Neurosci, 24(35), 7674-7680. doi:10.1523/JNEUROSCI.244104.2004

Herbert, H., Moga, M. M., \& Saper, C. B. (1990). Connections of the parabrachial nucleus with the nucleus of the solitary tract and the medullary reticular formation in the rat. J Comp Neurol, 293(4), 540-580. doi:10.1002/cne.902930404

Herdegen, T., \& Leah, J. D. (1998). Inducible and constitutive transcription factors in the mammalian nervous system: control of gene expression by Jun, Fos and Krox, and CREB/ATF proteins. Brain Res Brain Res Rev, 28(3), 370-490.

Hermann, G. E., \& Rogers, R. C. (1985). Convergence of vagal and gustatory afferent input within the parabrachial nucleus of the rat. J Auton Nerv Syst, 13(1), 1-17.

Hermanson, O., \& Blomqvist, A. (1997). Subnuclear localization of FOS-like immunoreactivity in the parabrachial nucleus after orofacial nociceptive stimulation of the awake rat. J Comp Neurol, 387(1), 114-123. 
Heyer, B. R., Taylor-Burds, C. C., Mitzelfelt, J. D., \& Delay, E. R. (2004). Monosodium glutamate and sweet taste: discrimination between the tastes of sweet stimuli and glutamate in rats. Chem Senses, 29(8), 721-729. doi:10.1093/chemse/bjh081

Hisatsune, C., Yasumatsu, K., Takahashi-Iwanaga, H., Ogawa, N., Kuroda, Y., Yoshida, R., . . Mikoshiba, K. (2007). Abnormal taste perception in mice lacking the type 3 inositol 1,4,5-trisphosphate receptor. J Biol Chem, $282(51), 37225-37231$. doi:10.1074/jbc.M705641200

Ikeda, K. (1909). New seasonings (in Japanese). J. Tokyo Chem. Soc., 30, 820-836.

Ikeda, K. (2002). New seasonings. Chem Senses, 27(9), 847-849.

Inoue, M., Beauchamp, G. K., \& Bachmanov, A. A. (2004). Gustatory neural responses to umami taste stimuli in C57BL/6ByJ and 129P3/J mice. Chem Senses, 29(9), 789-795. doi:10.1093/chemse/bjh083

Inoue, M., McCaughey, S. A., Bachmanov, A. A., \& Beauchamp, G. K. (2001). Whole nerve chorda tympani responses to sweeteners in C57BL/6ByJ and 129P3/J mice. Chem Senses, 26(7), 915-923.

Karimnamazi, H., \& Travers, J. B. (1998). Differential projections from gustatory responsive regions of the parabrachial nucleus to the medulla and forebrain. Brain Res, 813(2), 283-302.

Karimnamazi, H., Travers, S. P., \& Travers, J. B. (2002). Oral and gastric input to the parabrachial nucleus of the rat. Brain Res, 957(2), 193-206.

Kaufman, G. D. (2005). Fos expression in the vestibular brainstem: what one marker can tell us about the network. Brain Res Brain Res Rev, 50(1), 200-211. doi:10.1016/j.brainresrev.2005.06.001

Kawai, M., Okiyama, A., \& Ueda, Y. (2002). Taste enhancements between various amino acids and IMP. Chem Senses, 27(8), 739-745.

Keast, R. S., Canty, T. M., \& Breslin, P. A. (2004). The influence of sodium salts on binary mixtures of bitter-tasting compounds. Chem Senses, 29(5), 431-439. doi:10.1093/chemse/bjh045

Keskitalo, K., Knaapila, A., Kallela, M., Palotie, A., Wessman, M., Sammalisto, S., . . . Perola, M. (2007). Sweet taste preferences are partly genetically determined: identification of a trait locus on chromosome 16. Am J Clin Nutr, 86(1), 55-63.

Kiefer, S. W., \& Dopp, J. M. (1989). Taste reactivity to alcohol in rats. Behav Neurosci, 103(6), 1318-1326. 
Kiefer, S. W., Hill, K. G., \& Kaczmarek, H. J. (1998). Taste reactivity to alcohol and basic tastes in outbred mice. Alcohol Clin Exp Res, 22(5), 1146-1151.

King, M. S. (2007). Anatomy of the Rostral Nucleus of the Solitary Tract. In R. M. Bradley (Ed.), The Role of the Nucleus of the Solitary Tract in Gustatory Processing. Boca Raton (FL).

Krout, K. E., \& Loewy, A. D. (2000). Parabrachial nucleus projections to midline and intralaminar thalamic nuclei of the rat. J Comp Neurol, 428(3), 475-494.

Krukoff, T. L. (n.d.). C-fos expression as a marker of functional activity in the Brain: Immunohistochemistry. Cell Neurobiology Techniques, 33, 213-230. doi: $10.1385 / 0-89603-510-7: 213$

Kwak, Y., Han, J., Rhyu, M. R., Nam, T. S., Leem, J. W., \& Lee, B. H. (2015). Different spatial expressions of $c-F o s$ in the nucleus of the solitary tract following taste stimulation with sodium, potassium, and ammonium ions in rats. J Neurosci Res, 93(2), 340-349. doi:10.1002/jnr.23485

Landschulz, W. H., Johnson, P. F., \& McKnight, S. L. (1988). The leucine zipper: a hypothetical structure common to a new class of DNA binding proteins. Science, 240(4860), 1759-1764.

Lau, L. F., \& Nathans, D. (1987). Expression of a set of growth-related immediate early genes in BALB/c 3T3 cells: coordinate regulation with $c$-fos or c-myc. Proc Natl Acad Sci U S A, 84(5), 1182-1186.

Li, X. (2009). T1R receptors mediate mammalian sweet and umami taste. Am J Clin Nutr, 90(3), 733S-737S. doi:10.3945/ajen.2009.27462G

Li, X., Li, W., Wang, H., Cao, J., Maehashi, K., Huang, L., . . Brand, J. G. (2005). Pseudogenization of a sweet-receptor gene accounts for cats' indifference toward sugar. PLoS Genet, 1(1), 27-35. doi:10.1371/journal.pgen.0010003

Li, X., Staszewski, L., Xu, H., Durick, K., Zoller, M., \& Adler, E. (2002). Human receptors for sweet and umami taste. Proc Natl Acad Sci U S A, 99(7), 4692-4696. doi:10.1073/pnas.072090199

Lindemann, B. (2000). A taste for umami. Nat Neurosci, 3(2), 99-100. doi:10.1038/72153

Lumb, B. M., \& Morrison, J. F. (1987). An excitatory influence of dorsolateral pontine structures on urinary bladder motility in the rat. Brain Res, 435(1-2), 363-366.

Lundy, R. F., \& Norgren, R. (2004). Gustatory System. In G. Paxinos (Ed.), The Rat Nervous System (3rd ed., pp. 891-921). San Diego, CA: Elsevier Academic Press. 
Lush, I. E. (1984). The genetics of tasting in mice. III. Quinine. Genet Res, 44(2), 151160.

Lush, I. E. (1989). The genetics of tasting in mice. VI. Saccharin, acesulfame, dulcin and sucrose. Genet Res, 53(2), 95-99.

Max, M., Shanker, Y. G., Huang, L., Rong, M., Liu, Z., Campagne, F., . . Margolskee, R. F. (2001). Tas1r3, encoding a new candidate taste receptor, is allelic to the sweet responsiveness locus Sac. Nat Genet, 28(1), 58-63. doi:10.1038/88270

Mello, C. V., \& Pinaud, R. (2006). Immediate Early Gene Regulation in the Auditory System. In R. Pinaud \& L. A. Tremere (Eds.), Immediate Early Genes in Sensory Processing Cognitive Performance and Neurological Disorders (pp. 35-56). New York, NY: Springer Business Science Media, LLC.

Menendez, L., Bester, H., Besson, J. M., \& Bernard, J. F. (1996). Parabrachial area: electrophysiological evidence for an involvement in cold nociception. $J$ Neurophysiol, 75(5), 2099-2116.

Miura, H., Ooki, M., Kanemaru, N., \& Harada, S. (2014). Decline of umami preference in aged rats. Neurosci Lett, 577, 56-60. doi:10.1016/j.neulet.2014.06.018

Montag-Sallaz, M., Welzl, H., Kuhl, D., Montag, D., \& Schachner, M. (1999). Noveltyinduced increased expression of immediate-early genes $c$-fos and arg 3.1 in the mouse brain. J Neurobiol, 38(2), 234-246.

Montag-Sallaz, M., \& Montag, D. (2006). Immediate Early Genes and Sensory Maps of Olfactory and Gustatory Function. In R. Pinaud \& L. A. Tremere (Eds.), Immediate Early Genes in Sensory Processing, Cognitive Performance and Neurological Disorders (pp. 57-72). New York, NY: Springer Science Business Media, LLC.

Montmayeur, J. P., Liberles, S. D., Matsunami, H., \& Buck, L. B. (2001). A candidate taste receptor gene near a sweet taste locus. Nat Neurosci, 4(5), 492-498. doi:10.1038/87440

Morgan, J. I., \& Curran, T. (1986). Role of ion flux in the control of $c$-fos expression. Nature, 322(6079), 552-555. doi:10.1038/322552a0

Morgan, J. I., \& Curran, T. (1991). Stimulus-transcription coupling in the nervous system: involvement of the inducible proto-oncogenes fos and jun. Annu Rev Neurosci, 14, 421-451. doi:10.1146/annurev.ne.14.030191.002225

Morganti, J. M., Odegard, A. K., \& King, M. S. (2007). The number and location of Foslike immunoreactive neurons in the central gustatory system following electrical 
stimulation of the parabrachial nucleus in conscious rats. Chem Senses, 32(6), 543-555. doi:10.1093/chemse/bjm023

Mouritsen, O. G. (2013). The Taste of Seaweeds. Seaweeds: Edible, Available, and Sustainable (pp. 95-98). Hong Kong: The University of Chicago Press.

Muller, R., Bravo, R., Burckhardt, J., \& Curran, T. (1984). Induction of $c$-fos gene and protein by growth factors precedes activation of c-myc. Nature, 312(5996), 716720.

Nakashima, K., Eddy, M. C., Katsukawa, H., Delay, E. R., \& Ninomiya, Y. (2012). Behavioral responses to glutamate receptor agonists and antagonists implicate the involvement of brain-expressed mGluR4 and mGluR1 in taste transduction for umami in mice. Physiol Behav, 105(3), 709-719.

doi:10.1016/j.physbeh.2011.09.028

Nakashima, K., Katsukawa, H., Sasamoto, K., \& Ninomiya, Y. (2001). Behavioral taste similarities and differences among monosodium L-glutamate and glutamate receptor agonists in C57BL mice. J Nutr Sci Vitaminol (Tokyo), 47(2), 161-166.

Narukawa, M., Toda, Y., Nakagita, T., Hayashi, Y., \& Misaka, T. (2014). L-Theanine elicits umami taste via the T1R1 + T1R3 umami taste receptor. Amino Acids, 46(6), 1583-1587. doi:10.1007/s00726-014-1713-3

Nelson, G., Chandrashekar, J., Hoon, M. A., Feng, L., Zhao, G., Ryba, N. J., \& Zuker, C. S. (2002). An amino-acid taste receptor. Nature, 416(6877), 199-202. doi:10.1038/nature726

Nelson, G., Hoon, M. A., Chandrashekar, J., Zhang, Y., Ryba, N. J., \& Zuker, C. S. (2001). Mammalian sweet taste receptors. Cell, 106(3), 381-390.

Nishijo, H., Ono, T., \& Norgren, R. (1991). Parabrachial gustatory neural responses to monosodium glutamate ingested by awake rats. Physiol Behav, 49(5), 965-971.

Norgren, R. (1974). Gustatory afferents to ventral forebrain. Brain Res, 81(2), 285-295.

Norgren, R., \& Leonard, C. M. (1973). Ascending central gustatory pathways. J Comp Neurol, 150(2), 217-237. doi:10.1002/cne.901500208

Norgren, R., \& Pfaffmann, C. (1975). The pontine taste area in the rat. Brain Res, 91(1), 99-117.

Norgren, R., \& Wolf, G. (1975). Projections of thalamic gustatory and lingual areas in the rat. Brain Res, 92(1), 123-129. 
O'Doherty, J., Rolls, E. T., Francis, S., Bowtell, R., \& McGlone, F. (2001).

Representation of pleasant and aversive taste in the human brain. $J$ Neurophysiol, 85(3), 1315-1321.

Ohara, I., \& Naim, M. (1977). Effects of monosodium glutamate on eating and drinking behavior in rats. Physiol Behav, 19(5), 627-634.

Parker, L. A., Maier, S., Rennie, M., \& Crebolder, J. (1992). Morphine- and naltrexoneinduced modification of palatability: analysis by the taste reactivity test. Behav Neurosci, 106(6), 999-1010.

Perl, E., Shay, U., Hamburger, R.; and Steiner, J.E. (1992). Taste- and odor-reactivity in elderly demented patients. Chem Senses, 17(6), 779-794.

Rolls, B. J., Rowe, E. A., \& Rolls, E. T. (1982). How sensory properties of foods affect human feeding behavior. Physiol Behav, 29(3), 409-417.

Rolls, E. T. (2015). Taste, olfactory, and food reward value processing in the brain. Prog Neurobiol, 127-128, 64-90. doi:10.1016/j.pneurobio.2015.03.002

Rolls, E. T., \& Baylis, L. L. (1994). Gustatory, olfactory, and visual convergence within the primate orbitofrontal cortex. $J$ Neurosci, 14(9), 5437-5452.

Roncari, C. F., David, R. B., Johnson, R. F., De Paula, P. M., Colombari, D. S., De Luca, L. A., Jr., . . Menani, J. V. (2014). Angiotensinergic and cholinergic receptors of the subfornical organ mediate sodium intake induced by GABAergic activation of the lateral parabrachial nucleus. Neuroscience, 262, 1-8. doi:10.1016/j.neuroscience.2013.12.038

Roper, S. D. (2013). Taste buds as peripheral chemosensory processors. Semin Cell Dev Biol, 24(1), 71-79. doi:10.1016/j.semcdb.2012.12.002

Ruiz, C. J., Wray, K., Delay, E., Margolskee, R. F., \& Kinnamon, S. C. (2003). Behavioral evidence for a role of alpha-gustducin in glutamate taste. Chem Senses, 28(7), 573-579.

Sainz, E., Korley, J. N., Battey, J. F., \& Sullivan, S. L. (2001). Identification of a novel member of the T1R family of putative taste receptors. J Neurochem, 77(3), 896903.

Saites, L. N., Goldsmith, Z., Densky, J., Guedes, V. A., \& Boughter, J. D., Jr. (2015). Mice perceive synergistic umami mixtures as tasting sweet. Chem Senses, 40(5), 295-303. doi:10.1093/chemse/bjv010

San Gabriel, A., Uneyama, H., Yoshie, S., \& Torii, K. (2005). Cloning and characterization of a novel mGluR1 variant from vallate papillae that functions as 
a receptor for L-glutamate stimuli. Chem Senses, 30 Suppl 1, i25-26.

doi:10.1093/chemse/bjh095

Saper, C. B. (2004). Central Autonomic System. In G. Paxinos (Ed.), The Rat Nervous System (pp. 761-796). San Diego: Elsevier, Inc.

Saper, C. B., \& Loewy, A. D. (1980). Efferent connections of the parabrachial nucleus in the rat. Brain Res, 197(2), 291-317.

Schoenfeld, M. A., Neuer, G., Tempelmann, C., Schussler, K., Noesselt, T., Hopf, J. M., \& Heinze, H. J. (2004). Functional magnetic resonance tomography correlates of taste perception in the human primary taste cortex. Neuroscience, 127(2), 347353. doi:10.1016/j.neuroscience.2004.05.024

Schwarz, J., Burguet, J., Rampin, O., Fromentin, G., Andrey, P., Tome, D., . . . Darcel, N. (2010). Three-dimensional macronutrient-associated Fos expression patterns in the mouse brainstem. PLoS One, 5(2), e8974. doi:10.1371/journal.pone.0008974

Sclafani, A., Azzara, A. V., Touzani, K., Grigson, P. S., \& Norgren, R. (2001). Parabrachial nucleus lesions block taste and attenuate flavor preference and aversion conditioning in rats. Behav Neurosci, 115(4), 920-933.

Shimura, T., Tokita, K., \& Yamamoto, T. (2002). Parabrachial unit activities after the acquisition of conditioned taste aversion to a non-preferred $\mathrm{HCl}$ solution in rats. Chem Senses, 27(2), 153-158.

Slugg, R. M., \& Light, A. R. (1994). Spinal cord and trigeminal projections to the pontine parabrachial region in the rat as demonstrated with Phaseolus vulgaris leucoagglutinin. J Comp Neurol, 339(1), 49-61. doi:10.1002/cne.903390106

Small, D. M., Jones-Gotman, M., Zatorre, R. J., Petrides, M., \& Evans, A. C. (1997). Flavor processing: more than the sum of its parts. Neuroreport, 8(18), 3913-3917.

Smith, D. V., \& Boughter, J. D. (2007). Neurochemistry of the Gustatory System. In D. Johnson \& A. Lajtha (Eds.), Handbook of Neurochemistry and Molecular Neurobiology: Sensory Neurochemistry (3rd ed., pp. 109-135). Berlin: Springer US.

Spector, A. C. (2000). Linking gustatory neurobiology to behavior in vertebrates. Neurosci Biobehav Rev, 24(4), 391-416.

Spector, A. C., Breslin, P., \& Grill, H. J. (1988). Taste reactivity as a dependent measure of the rapid formation of conditioned taste aversion: a tool for the neural analysis of taste-visceral associations. Behav Neurosci, 102(6), 942-952. 
Spector, A. C., \& Glendinning, J. I. (2009). Linking peripheral taste processes to behavior. Curr Opin Neurobiol, 19(4), 370-377. doi:10.1016/j.conb.2009.07.014

St. John, S. J., \& Spector, A. C. (2008). Behavioral Analysis of Taste Function in Rodent Models. In A. I. Basbaum, A. Kaneko, G. M. Shepard, \& G. Westheimer (Eds.), The Senses: A Comprehensive Reference: Olfaction and Taste (Vol. 4, pp. 409428). San Diego, CA: Academic Press.

Stapleton, J. R., Luellig, M., Roper, S. D., \& Delay, E. R. (2002). Discrimination between the tastes of sucrose and monosodium glutamate in rats. Chem Senses, 27(4), 375-382.

Stapleton, J. R., Roper, S. D., \& Delay, E. R. (1999). The taste of monosodium glutamate (MSG), L-aspartic acid, and N-methyl-D-aspartate (NMDA) in rats: are NMDA receptors involved in MSG taste? Chem Senses, 24(4), 449-457.

Steiner, J. E. (1973). The gustofacial response: observation on normal and anencephalic newborn infants. Symp Oral Sens Percept(4), 254-278.

Steiner, J. E. (1974). Discussion paper: innate, discriminative human facial expressions to taste and smell stimulation. Ann N Y Acad Sci, 237(0), 229-233.

Steiner, J. E. (1979). Human facial expressions in response to taste and smell stimulation. Adv Child Dev Behav, 13, 257-295.

Steiner, J. E. (1987). What the Neonate Can Tell Us About Umami. In Y. Kawamura \& M. R. Kare (Eds.), Umami: A Basic Taste: Physiology, Biochemistry, Nutrition, Food Science (pp. 97-123). New York, NY: Marcel Dekker.

Steiner, J. E., Glaser, D., Hawilo, M. E., \& Berridge, K. C. (2001). Comparative expression of hedonic impact: affective reactions to taste by human infants and other primates. Neurosci Biobehav Rev, 25(1), 53-74.

Steiner, J. E., Glaser D. (1984). Differential behavioral responses to taste stimuli in non-human primates. Journal of Human Evolution, 13(8), 709-723.

Steiner, J. E., Glaser D. (1995). Taste-induced facial expressions in apes and humans. Human Evolution, 10(2), 97-105.

Sugita, M., \& Shiba, Y. (2005). Genetic tracing shows segregation of taste neuronal circuitries for bitter and sweet. Science, 309(5735), 781-785. doi:10.1126/science.1110787

Terleph, T., \& Tremere, L. A. (2006). The Use of Immediate Early Genes as Mapping Tools for Neuronal Activation: Concepts and Methods. In R. Pinaud \& L. A. Tremere (Eds.), Immediate Early Genes in Sensory Processing, Cognitive 
Performance and Neurological Disorders (pp. 1-10). New York, NY: Springer Science Business Media, LLC.

Tokita, K., Armstrong, W. E., St John, S. J., \& Boughter, J. D., Jr. (2014). Activation of lateral hypothalamus-projecting parabrachial neurons by intraorally delivered gustatory stimuli. Front Neural Circuits, 8, 86. doi:10.3389/fncir.2014.00086

Tokita, K., \& Boughter, J. D., Jr. (2012). Sweet-bitter and umami-bitter taste interactions in single parabrachial neurons in C57BL/6J mice. J Neurophysiol, 108(8), 21792190. doi: $10.1152 /$ jn. 00465.2012

Tokita, K., \& Boughter, J. D., Jr. (2016). Topographic organizations of taste-responsive neurons in the parabrachial nucleus of C57BL/6J mice: An electrophysiological mapping study. Neuroscience, 316, 151-166.

doi:10.1016/j.neuroscience.2015.12.030

Tokita, K., Inoue, T., \& Boughter, J. D., Jr. (2009). Afferent connections of the parabrachial nucleus in C57BL/6J mice. Neuroscience, 161(2), 475-488. doi:S0306-4522(09)00448-5 [pii] 10.1016/j.neuroscience.2009.03.046

Tokita, K., Yamamoto, T., \& Boughter, J. D., Jr. (2012). Gustatory neural responses to umami stimuli in the parabrachial nucleus of C57BL/6J mice. J Neurophysiol, 107(6), 1545-1555. doi:10.1152/jn.00799.2011

Tordoff, M. G. (2007). Taste solution preferences of C57BL/6J and 129X1/SvJ mice: influence of age, sex, and diet. Chem Senses, 32(7), 655-671. doi:10.1093/chemse/bjm034

Tordoff, M. G., \& Bachmanov, A. A. (2002). Influence of test duration on the sensitivity of the two-bottle choice test. Chem Senses, 27(9), 759-768.

Toyono, T., Seta, Y., Kataoka, S., Harada, H., Morotomi, T., Kawano, S., . . Toyoshima, K. (2002). Expression of the metabotropic glutamate receptor, mGluR4a, in the taste hairs of taste buds in rat gustatory papillae. Arch Histol Cytol, 65(1), 91-96.

Toyono, T., Seta, Y., Kataoka, S., Kawano, S., Shigemoto, R., \& Toyoshima, K. (2003). Expression of metabotropic glutamate receptor group I in rat gustatory papillae. Cell Tissue Res, 313(1), 29-35. doi:10.1007/s00441-003-0740-2

Travers, J. B., Herman, K., Yoo, J., \& Travers, S. P. (2007). Taste reactivity and Fos expression in GAD1-EGFP transgenic mice. Chem Senses, 32(2), 129-137. doi:10.1093/chemse/bj1038 
Travers, J. B., Urbanek, K., \& Grill, H. J. (1999). Fos-like immunoreactivity in the brain stem following oral quinine stimulation in decerebrate rats. Am J Physiol, 277(2 Pt 2), R384-394.

Travers, S. P., \& Hu, H. (2000). Extranuclear projections of rNST neurons expressing gustatory-elicited Fos. J Comp Neurol, 427(1), 124-138.

Van Der Gucht, E., Vandenbussche, E., Orban, G. A., Vandesande, F., \& Arckens, L. (2000). A new cat Fos antibody to localize the immediate early gene $c$-fos in mammalian visual cortex after sensory stimulation. J Histochem Cytochem, 48(5), 671-684.

Vandenbeuch, A., \& Kinnamon, S. C. (2009). Why do taste cells generate action potentials? J Biol, 8(4), 42. doi:10.1186/jbiol138

Vigues, S., Dotson, C. D., \& Munger, S. D. (2009). The receptor basis of sweet taste in mammals. Results Probl Cell Differ, 47, 187-202.doi:10.1007/400_2008_2

Waldern, D. E., \& Van Dyk, R. D. (1971). Effect of Monosodium Glutamate in Starter Rations on Feed Consumption and Performance of Early Weaned Calves. Journal of Dairy Science, 54(2), 262-265. doi:10.3168/jds.s0022-0302(71)85822-8

Yamaguchi, S. (1991). Basic properties of umami and effects on humans. Physiol Behav, 49(5), 833-841.

Yamamoto, T., Matsuo, R., Fujimoto, Y., Fukunaga, I., Miyasaka, A., \& Imoto, T. (1991). Electrophysiological and behavioral studies on the taste of umami substances in the rat. Physiol Behav, 49(5), 919-925.

Yamamoto, T., Matsuo, R., Kiyomitsu, Y., \& Kitamura, R. (1988). Taste effects of 'umami' substances in hamsters as studied by electrophysiological and conditioned taste aversion techniques. Brain Res, 451(1-2), 147-162.

Yamamoto, T., \& Sawa, K. (2000). Comparison of $c$-fos-like immunoreactivity in the brainstem following intraoral and intragastric infusions of chemical solutions in rats. Brain Res, 866(1-2), 144-151.

Yamamoto, T., Shimura, T., Sakai, N., \& Ozaki, N. (1994). Representation of hedonics and quality of taste stimuli in the parabrachial nucleus of the rat. Physiol Behav, 56(6), 1197-1202.

Yamamoto, T., Shimura, T., Sako, N., Sakai, N., Tanimizu, T., \& Wakisaka, S. (1993). $C$-fos expression in the parabrachial nucleus after ingestion of sodium chloride in the rat. Neuroreport, 4(11), 1223-1226. 
Yamamoto, T., Takemura, M., Inui, T., Torii, K., Maeda, N., Ohmoto, M., ... Abe, K. (2009). Functional organization of the rodent parabrachial nucleus. Ann N Y Acad Sci, 1170, 378-382. doi:10.1111/j.1749-6632.2009.03883.x

Yamamoto, T., Yuyama, N., Kato, T., \& Kawamura, Y. (1985a). Gustatory responses of cortical neurons in rats. II. Information processing of taste quality. $J$ Neurophysiol, 53(6), 1356-1369.

Yasumatsu, K., Horio, N., Murata, Y., Shirosaki, S., Ohkuri, T., Yoshida, R., \& Ninomiya, Y. (2009). Multiple receptors underlie glutamate taste responses in mice. Am J Clin Nutr, 90(3), 747S-752S. doi:10.3945/ajen.2009.27462J

Yoshimura, H., Sugai, T., Fukuda, M., Segami, N., \& Onoda, N. (2004). Cortical spatial aspects of optical intrinsic signals in response to sucrose and $\mathrm{NaCl}$ stimuli. Neuroreport, 15(1), 17-20.

Zatorre, R. J., Jones-Gotman, M., Evans, A. C., \& Meyer, E. (1992). Functional localization and lateralization of human olfactory cortex. Nature, 360(6402), 339340. doi:10.1038/360339a0

Zhang, Y., Hoon, M. A., Chandrashekar, J., Mueller, K. L., Cook, B., Wu, D., . . Ryba, N. J. (2003). Coding of sweet, bitter, and umami tastes: different receptor cells sharing similar signaling pathways. Cell, 112(3), 293-301. 


\section{VITA}

Jennifer Marie Saputra was born in New York, New York in 1984. She was raised in quite a few cities in the country but spent the most time in the Memphis area. She graduated from Bolton High school in May 2002. She then attended Christian Brothers University in Memphis, TN and graduated cum laude in May 2006 with a Bachelor of Science degree in Biology with minors in Behavioral Sciences and Chemistry. While at CBU, she was selected to go to Florianopolis, Brazil to perform her senior research project during the summer of 2005 with the MHIRT program. This opportunity helped her to realize that she wanted to attend graduate school. In 2006, she started in the IPBS program at the University of Tennessee Health Science Center in Memphis, TN and chose the Neuroscience Track. While in graduate school she was given many opportunities to present her research at various conferences around the country. She was also given many opportunities to teach lectures at both UTHSC and Christian Brothers University and was an adjunct lecturer for Anatomy and Physiology II lab for a semester. 